\title{
Seismic Monitoring in the Framework of the Pilot Project CLEAN
}

\author{
Inauguraldissertation \\ zur Erlangung des Grades eines \\ Doktors der Naturwissenschaft \\ am Fachbereich Geowissenschaften \\ der Freien Universität Berlin
}

vorgelegt von

Lars Houpt

Berlin 2012 

Erstgutachter: Prof. Dr. Serge A. Shapiro

Zweitgutachter: Prof. Dr. Stefan Buske

Datum der Disputation: 2. November 2012 



\section{Contents}

1 Introduction $\quad 1$

1.1 Motivation . . . . . . . . . . . . . . . . . 4

$\begin{array}{lll}2 & \text { Test-site } & 7\end{array}$

2.1 The investigation area . . . . . . . . . . . . . . . . . . . 9

2.1.1 The Central European Basin System (CEBS) . . . . . . . . 9

2.1.2 The Altmark gas field . . . . . . . . . . . . . . . . . . 11

$2.2 \mathrm{MSP} / \mathrm{VSP}$ (moving source profiling/ vertical seismic profiling) . . . . 14

2.3 Other $\mathrm{CO}_{2}$ sequestration projects . . . . . . . . . . . . . 15

3 Modelling of the seismic wavefield 21

3.1 Theory . . . . . . . . . . . . . . . . . . . 21

3.1.1 Viscoelasticity . . . . . . . . . . . . . . . . 22

3.1.2 Viscoelastic wave equations . . . . . . . . . . . . . 24

3.1.3 Finite-difference method . . . . . . . . . . . . . . 26

3.2 The program fdveps_mpi and modeling parameters . . . . . . . . . . 29

3.2.1 FD-order . . . . . . . . . . . . . . . . . . . . . . . . 29

3.2 .2 Parallelization . . . . . . . . . . . . . . . . 29

3.2 .3 Model . . . . . . . . . . . . . . . . . . . . . . . . 29

3.2.4 Attenuation ....................... 31

3.2 .5 Boundaries ......................... 31

3.2 .6 Source . . . . . . . . . . . . . . . . . . . . . 32

3.2 .7 Receiver . . . . . . . . . . . . . . . . 32

3.3 Preprocessing . . . . . . . . . . . . . . . . . . . . . 32

3.3.1 Static shift . . . . . . . . . . . . . . 33

3.3 .2 Wavefield separation . . . . . . . . . . . . . . 33

3.3 .3 Noise . . . . . . . . . . . . . . . . . . . . . . 36

4 Amplitude changes $\quad 39$

4.1 Influence of rock properties . . . . . . . . . . . . . . . . . . . . 40 
4.2 Propagation of the $\mathrm{CO}_{2}$-front . . . . . . . . . . . . . . . . . . . . . . . . . . . . . . .

4.3 Source-receiver offset . . . . . . . . . . . . . . . . . . . . . . . 44

4.4 Noise . . . . . . . . . . . . . . . . . . . . . . . 44

4.5 Shift . . . . . . . . . . . . . . . . . 46

4.6 Conclusion . . . . . . . . . . . . . . . . . . . . . . . 48

5 Migration $\quad 51$

5.1 Theory . . . . . . . . . . . . . . . . . . 51

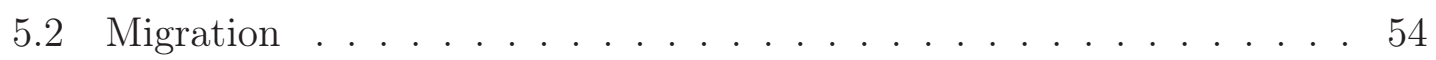

5.2 .1 Kirchhoff migration . . . . . . . . . . . . 55

5.2 .2 Fresnel-Volume migration . . . . . . . . . . . 56

5.3 Noise . . . . . . . . . . . . . . . . . . . . . . . . 57

5.4 Shift . . . . . . . . . . . . . . . . . 57

5.5 Source density . . . . . . . . . . . . . . . . . . . . . . . 59

5.6 Conclusion . . . . . . . . . . . . . . . . . . . . . . . . . 59

6 Coda wave interferometry 63

6.1 Theory . . . . . . . . . . . . . . . . . . 63

6.2 Tests . . . . . . . . . . . . . . . . . . . . 64

6.2 .1 Maximum time shift $t_{s, \max } \ldots \ldots \ldots . \ldots 65$

6.2.2 Length of the time window $2 t_{w} \ldots \ldots . \ldots 68$

6.3 Application to the Altmark-model . . . . . . . . . . . . . . . . . 70

6.3.1 Velocity changes . . . . . . . . . . . . 70

6.3.2 Propagation . . . . . . . . . . . . . . . 72

6.3 .3 Source-receiver offset . . . . . . . . . . . . . . . 74

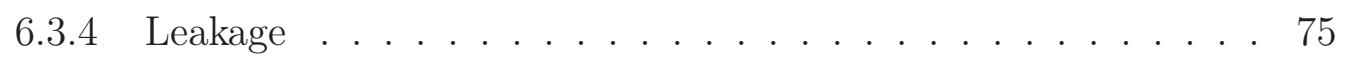

6.3 .5 Noise . . . . . . . . . . . . . . . . 76

6.3 .6 Shift ......................... 77

6.4 Conclusion . . . . . . . . . . . . . . . . . . . . 78

7 Rock Physics $\quad 81$

7.1 Gas properties . . . . . . . . . . . . . . . . 81

7.2 Saturated rock properties . . . . . . . . . . . . . . 84

7.3 Conclusion . . . . . . . . . . . . . . . . . . . . . . . 88

$\begin{array}{llr}8 & \text { Discussion } & 89\end{array}$

$\begin{array}{ll}\text { Bibliography } & 95\end{array}$

$\begin{array}{ll}\text { A Comparison of equations of state } & 103\end{array}$ 


\section{Chapter 1}

\section{Introduction}

With the ratification and the commencement of the Kyoto Protocol on February 16 2005, Germany and 192 other nations engaged to reduce their greenhouse gas emissions. These anthropogenic emissions partly accumulate in the atmosphere and cause the climate change with an increase of the global temperature. The main greenhouse gases are carbon dioxide $\left(\mathrm{CO}_{2}\right)$, methane $\left(\mathrm{CH}_{4}\right)$, nitrous oxide $\left(\mathrm{N}_{2} \mathrm{O}\right)$ and fluorocarbons (HFC, PFC). Based on the Global Warming Potential (GWP100), $\mathrm{CO}_{2}$ contributes the largest amount to the climate change with about $80 \%$ for the Annex I countries (UNFCCC, 2011). The $\mathrm{CO}_{2}$-concentration in the atmosphere add up to about $372 \mathrm{ppm}$ in 2004 and reached the highest level within the last 420,000 years (King, 2004). To avoid a further increase above the critical value of $450 \mathrm{ppm}$ in the next decades, where experts believe that severe consequences can not be averted any more, politics, industry and people of all countries have to act.

The most obvious solutions are to reduce the consumption of energy by an increase of the efficiency of energy use and the optimization of power plant and industrial facilities. Also a stop of forest clearance and reforestation would sequest large amounts of $\mathrm{CO}_{2}$ in terrestrial ecosystems. Further a turn in the energy supply towards regenerative energies is necessary. However to put all these steps into practice is time and cost intensive. Other transitional technologies are necessary to span the time of modification. As one of the largest global carbon emitters and due to the regulation of $\mathrm{CO}_{2}$ emissions in Europe (ecological tax reforms) (Welfens et al., 1999), the greenhouse gas emission trading obligations for German energy industry since 2005 (Bode, 2003), and the phasing out of nuclear energy, Germany has special interest in transitional technologies. One strategy is obviating the emission of $\mathrm{CO}_{2}$ into the atmosphere by capturing it from power plants and big industrial complexes and storing it in other potential sinks. The most widely discussed sinks are the deep oceans, advanced chemical bonding of $\mathrm{CO}_{2}$, coal seams, deep saline aquifers and depleted gas and oil reservoirs. 
Of those sinks, the oceans exhibit the largest capacity. The injection could be direct by injecting $\mathrm{CO}_{2}$ via pipeline or from a ship in a depth of more than $3-4 \mathrm{~km}$, where the $\mathrm{CO}_{2}$ would, due to its density, sink to the ground and accumulate at the ocean bottom. Also an indirect injection would be possible by manuring the ocean. This activates the growth of alga and plankton and thus bonds $\mathrm{CO}_{2}$ in biomass (Anderson and Newell, 2003). Once died these particles sink to the ocean bottom. However the impact of a lowering of the ph-value, which is linked to a higher $\mathrm{CO}_{2}$ content, and of $\mathrm{CO}_{2}$ accumulations at the ocean bottom to the local biota especially in long term are not known (Johnston and Santillo, 2002).

The chemical storage of $\mathrm{CO}_{2}$ is based on the exothermal reaction of $\mathrm{CO}_{2}$ with silicates that gives Carbonates. This method is the only one, that guarantees a permanent storage of the $\mathrm{CO}_{2}$. However for this reaction large amounts of silicates are necessary, that are not always available and have to be mined, most likely in open-cast mines which again impairs the environment (Radgen et al., 2006).

There are generally two methods for storing $\mathrm{CO}_{2}$ in coal seams. The first is the storage in given up coal mines, that was already used for the short term storage of natural gas (e.g. in Belgium, Radgen et al., 2006). However due to the large amount of combined mines on her territory this technology is not practical in Germany. The second method is the injection in deep not-minable coal seams. This could be combined with the production of methane as the $\mathrm{CO}_{2}$ will be absorbed at the coal surface under simultaneous desorption of methane (White et al., 2003). This process is known as $\mathrm{CO}_{2}$ Enhanced Coal Bed Methane Recovery (CO2-ECBM), which is quite promising, as large $\mathrm{CO}_{2}$ emitters (heavy industry) are often located in the vicinity of coal deposits and far transportation of the captured $\mathrm{CO}_{2}$ could be avoided (fig. 1.1).

The most promising method though is the injection in deep saline aquifers, as those are available in nearly every sedimentary basin worldwide and are estimated to have the largest capacity after the oceans. To guarantee high effectiveness, the $\mathrm{CO}_{2}$ has to be injected in supercritical state, which is given in depths larger than $800 \mathrm{~m}$ (this counts also for the other injection methods). The $\mathrm{CO}_{2}$ would replace and afterwards slowly go into solution in the formation water. Therefore sealing layers are necessary to keep the $\mathrm{CO}_{2}$ in the aquifer long enough. Those barriers usually exist in most basins, separating the deeper saline aquifers from the shallower drinking water aquifers. Afterwards the $\mathrm{CO}_{2}$ would react with silicates from the rock matrix and would be stably stored in carbonates as explained for the chemical storage above. However this reaction is very slow and the $\mathrm{CO}_{2}$ would have to be stored in the aquifer for approximately 10,000 years. If the barrier rocks are not completely closed though, the $\mathrm{CO}_{2}$ could not be held in the aquifer long enough or the salt 


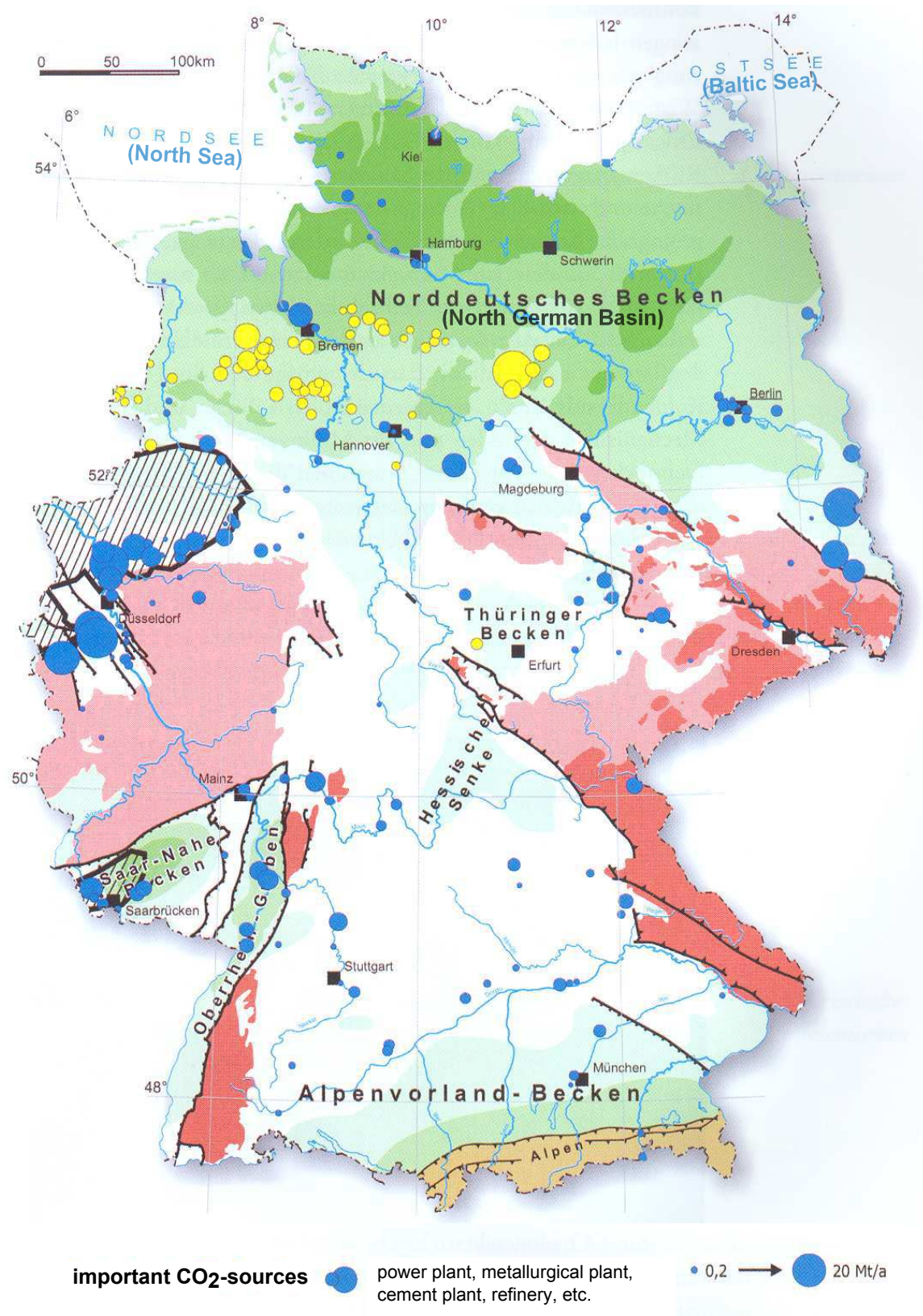

regions with storage potential

\section{regions without important storage potential}

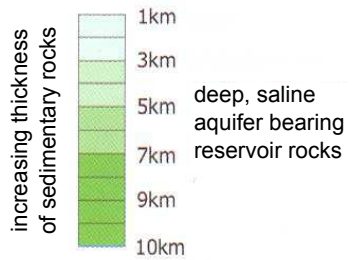

$\square$ metamorphic rocks

magmatic and highmetamorphic rocks

no reservoir rocks existing or in too great depth

\section{T/1/, coal seams \\ gas fields}

Figure 1.1: Distribution of $\mathrm{CO}_{2}$ emitters and storage sites in Germany (edited, Bundesanstalt für Geowissenschaften und Rohstoffe (BGR) in Hannover, http://www.sk-zag.de/3.5_CCS.html). 
water might be displaced into upper layers impurifying the drinking water aquifers (White et al., 2003; Radgen et al., 2006). However saline aquifers have the largest capacity in Germany with 6.3 - 12.8 billion t of $\mathrm{CO}_{2}$ (Reinhold et al., 2011).

Besides this, the cumulative storage capacity in German's oil and gas reservoirs of only 2.75 billion t of $\mathrm{CO}_{2}$ is comparatively small (Gerling, 2008). Nevertheless this technique has the advantage that those reservoirs have already proven their ability to store gases over a large time span and the geological settings are usually well known already. Also a large part of infrastructure (pipelines, wells etc.) already exist and can be used for the transport and injection of the $\mathrm{CO}_{2}$ (Rebscher and Oldenburg, 2005). Like for the coal seams the injection can be combined with enhanced oil and gas recovery (EOR and EGR) by increasing the reservoir pressure and displacing the remaining oil or gas towards the production well. This would make the injection more cost effective and it seems to be reasonable under considering that the global demand for fossil fuels will further increase in the next decades and between $67 \%$ and $72 \%$ of today's gas production worldwide originates from oil and gas fields that already passed their peak production (Rückheim et al., 2005).

However storage permanence has to be assured which means that $99 \%$ of the injected $\mathrm{CO}_{2}$ has to remain confined within the next 100 years (NETL, 2007). This of course can only be verified with reliable and highly accurate monitoring techniques. These include geophysical monitoring and modelling at reservoir depth, tracer, soil gas and groundwater monitoring, reservoir pressure and temperature monitoring and flow as well as geomechanical simulations at near- and far-field conditions continuously updated by feedback data (Sayers and Wilson, 2010). All of which were included in the joint research pilot project CLEAN $\left(\mathrm{CO}_{2}\right.$ Large-scale EGR in the Altmark Natural-gas field) amongst other (chemical and biological monitoring, etc.).

\subsection{Motivation}

Seismic experiments are the only possibility to monitor spatial and temporal changes of the rock physical properties in the reservoir and adjacent formations with high spatial resolution and thus are indispensable for each $\mathrm{CO}_{2}$ sequestration project. The present study is part of such a project and deals with the feasibility and prospects of active seismic monitoring. As the CLEAN project is accompanying an EGR project, the expected changes in the seismic wavefield are small such that the use of seismic borehole experiments with receivers as near as possible to the target zone and an optimized setup will increase the prospects of success. In order to guarantee an optimal setup and to appraise the chances of success, it is necessary to estimate the changes in reflection amplitudes, time shifts and their depth of occurrence. These 
estimations were performed in the framework of this dissertation on the basis of simulations of the seismic wavefield.

Therefore, after giving an overview of the CLEAN project, the test site, the planned seismic monitoring program and a comparison to other $\mathrm{CO}_{2}$ sequestration projects (chapter 2) is given. The modelling program with its parameters and velocity models and a few preprocessing steps applied to the data are explained in chapter 3. Chapter 4 and 5 show the results of the analysis of amplitude changes and depth migrations. Chapter 6 presents the results of the attempt to detect $\mathrm{CO}_{2}$ accumulations with coda-wave interferometry. Rock physical estimates are presented in chapter 7, giving an idea of the prospects of success of the seismic experiments in the Altmark. Finally the results are summarized giving a comprehensive survey plan for the active seismic monitoring attending a $\mathrm{CO}_{2}$-injection in the Altmark in chapter 8 . 



\section{Chapter 2}

\section{Test-site}

The joint research project CLEAN $\left(\mathrm{CO}_{2}\right.$ Large-scale EGR in the Altmark Naturalgas field) is a research and development $(\mathrm{R} \& \mathrm{D})$ program of altogether 16 scientific institutions and commercial enterprises of Germany and was founded over a period of three years, from July 2008 to June 2011, under the key area "Technologies for a Sustainable Storage of Carbon Dioxide in Geological Formations" of the GEOTECHNOLOGIEN program of the German Federal Ministry of Education and Research (BMBF). It covers the scientific program accompanying the Enhanced Gas Recovery (EGR) project accomplished by GDF SUEZ E\&P Deutschland GmbH (GDF SUEZ) and Vattenfall Europe within the second largest natural-gas field in Europe, the Altmark gas field (Kühn et al., 2011, 2012).

The goal of the CLEAN project was to research opportunities for the mobilization of natural gas volumes that are not conventionally extractable and to increase knowledge about the geological storage of $\mathrm{CO}_{2}$ in nearly exhausted natural gas fields. The scientific focus was to develop and optimize suitable technologies and methods

- for an optimum $\mathrm{CO}_{2}$ injection to achieve a maximum gas recovery,

- for the technical monitoring of the $\mathrm{CO}_{2}$ propagating in the lithosphere under consideration of environmentally relevant processes,

- for the realization of a long-term wellbore sealing,

- for the identification and assessment of the wellbore integrity of old wells, and

- the examination, description and assessment of all processes that are related to the $\mathrm{CO}_{2}$ injection and the displacement of the reservoir gas.

The structural framework of the CLEAN research project consists of five Thematic Networks: 
- pilot plant for $\mathrm{CO}_{2}$ injection,

- well bore integrity,

- geoscientific processes,

- environment and process monitoring,

- public acceptance.

Most of them are subdivided into further projects (CLEAN-webpage: http://www.cleanaltmark.org).

The work presented in this study was carried out within the "reservoir and cap-rock monitoring" as part of the "environment and process monitoring". The objective of this Thematic Network was to develop and test monitoring methods and to derive an environment and operation monitoring concept for the reservoir, the overlying rocks and the near-surface aquifer systems and the water-unsaturated soil zone. Beside geophysical methods (seismic, pressure, temperature) it comprises chemical (analyses of fluid and gas samples) as well as microbiological monitoring methods.

The seismic methods are divided into an active and a passive seismic survey, which are designed to monitor the spatial and temporal evolution of the $\mathrm{CO}_{2}$ front within the reservoir as well as the related processes. These experiments comprise a time-lapse VSP/MSP survey as well as the installation of a borehole seismometer network for the monitoring and analysis of potential injection-induced seismicity.

Political influences as well as the delay in the implementation of the directive of the European Parliament and Council about the geological storage of $\mathrm{CO}_{2}$ (DIRECTIVE 2009/31/EC) into German law resulted in a delay of the approval process and no permission for the injection of $\mathrm{CO}_{2}$ was given during the project period. This affected especially the geophysical monitoring. While the methods for the passive seismic monitoring and the pressure and temperature monitoring could be tested successfully at other locations, the active seismic monitoring could not be accomplished as planned. The focus was changed and the study of 2D-FD simulations of the seismic wavefield which were initially planned only in preparation of the VSP/MSP experiments were extended. The results provide important information for the realization and prospects of the VSP/MSP experiments, the acquisition geometry as well as the dimension of injection induced changes in reflection amplitudes in the seismic wavefield and for their detectability. They are documented in the present thesis. 


\subsection{The investigation area}

The investigation area of the CLEAN project is a hydrodynamically sealed compartment of the Altmark Gas Field in the federal state of Saxony-Anhalt, Germany. The Altmark Gas Field is located in the North German Basin which is part of the Central European Basin System (CEBS) that covers an area from the North Sea to Poland and from Norway to the German midlands. The CEBS is generally characterized by lowlands (Dutch, German and Danish Lowlands and shallow bathymetry in the relative parts of the North and Baltic Sea). To understand the behaviour of the gas field during injection and potential difficulties for the processing and interpretation of seismic data in this region an overview of the formation and geology of the basin and the gas field is given. Then the Altensalzwedel reservoir is presented in more detail.

\subsubsection{The Central European Basin System (CEBS)}

The crustal rheology below the CEBS is complex and consists of different crustal domains, Baltica that is of Praecambrian age and the Caledonian-Variscan Europe that was consolidated in the Palaeozoic era. Baltica comprises the East European Craton and the Baltic Shield that is outcropped at the northern margin of the CEBS in South Norway, South Sweden and on Bornholm Island. This arching occurred during the Caledonian Orogeny (500 - $400 \mathrm{Ma}$ ), when the subcontinents Laurentia and Avalonia accreted to Baltica forming Laurussia. The Caledonides (Avalonia and parts of Laurentia) are outcropped in the northern part of the British Isles and the Scandinavian Mountains flanking the CEBS in the North and West. The Variscides limit the basin towards the South with outcrops in the Rhenish Slate Mountains, the Harz Mountains and the Sudetes. They were formed during the Variscan Orogeny in the late Palaeozoic (400 - 320 Ma) when Gondwana and Laurussia collided constituting the supercontinent Pangaea.

During the transition from the Carboniferous to the Permian and later in the Triassic, during the break-up of Pangaea, the CEBS was affected by a number of pulses of tectonic extension and rifting that lead to the formation of superimposed subbasins which are the Northern Permian Basin (NPB), the Southern Permian Basin (SPB, containing the Southern North Sea and the North German Basin) and the Polish Trough (PT). Compressions and inversions in some basins occurred during the Alpine Orogeny in the latest Cretaceous when Africa-Arabia collided with Eurasia causing deep reaching faults and flexures.

Within the CEBS these crystalline basements are covered by Cambrian (even though not investigated by drilling they can be assumed) to Cenozoic sediments of 
up to 10,000 $\mathrm{m}$ thickness (Hamburger Trough, fig. 2.1). In the Late Carboniferous (Westphalian) and Early Permian (Rotliegend) the CEBS was located near the equator where tropical conditions led to the formation of coal seams that are source rocks for the hydrocarbon gas deposits accumulated in the sediments of the Carboniferous and Rotliegend (Schröder, 1989). The varying methane content within the basin region depends on the presence and maximum depth of the Carboniferous gas source rocks. Strong volcanism during the Variscan Orogeny led to thick series of rheolite and ignimbrite in the Rotliegend (Early Permian). They indicate that the crust was partially melted and they caused an increase of the rock density in the lower crust. During the Permian the CEBS moved northwards entering the arid zone where oxidized (red) clastics and salt dominate. During the Late Permian (Zechstein), the basin connected to the open sea and was flooded several times, still under arid conditions, leading to several evaporite cycles. This caused thick salt accumulations of up to $2000 \mathrm{~m}$ thickness in the center of the basin. They form the sealing cap rock for the gas deposits in the CEBS.

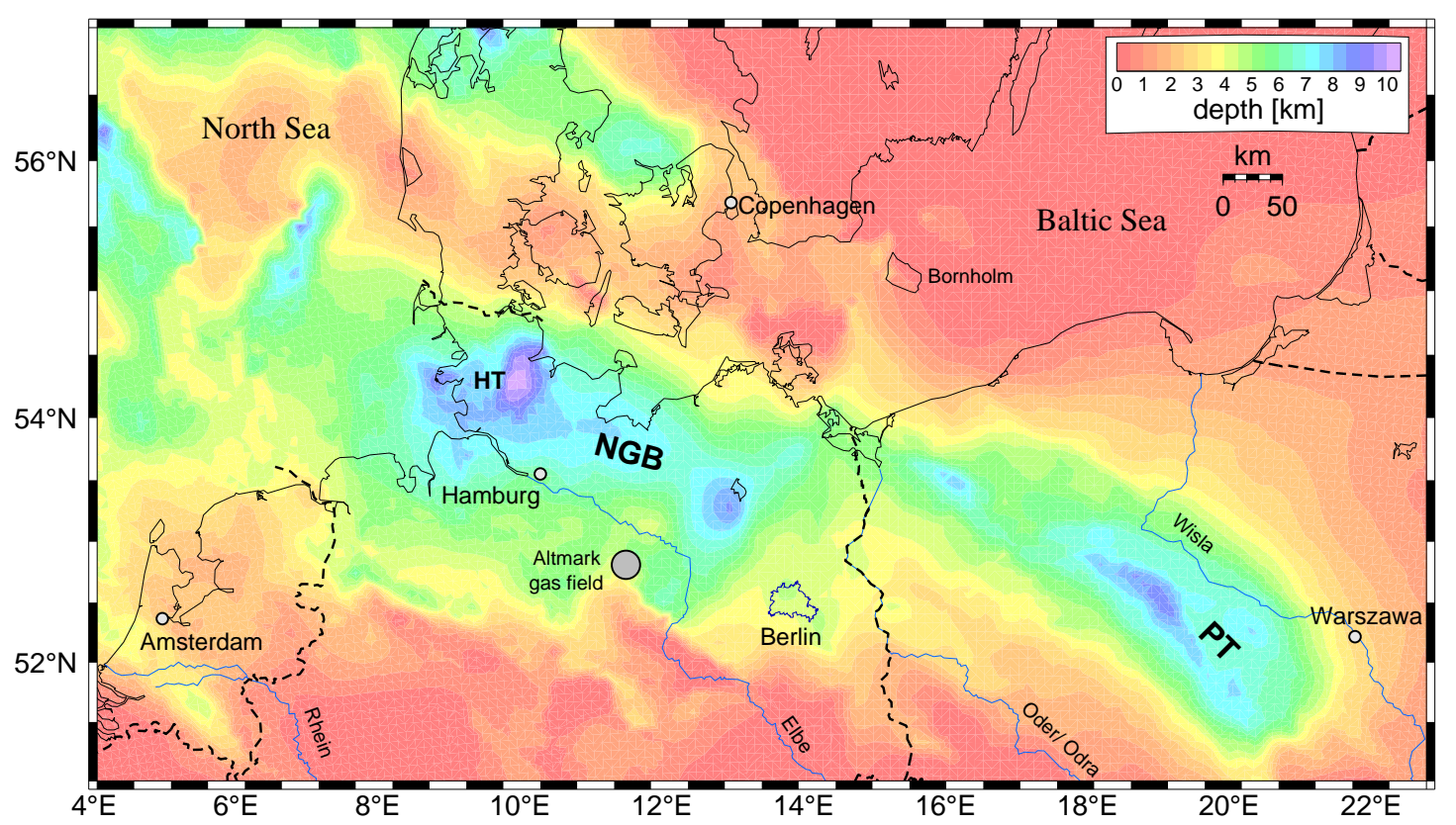

Figure 2.1: The base of the Permian in the region of the CEBS (based on a compilation of Scheck-Wenderoth and Lamarche (2005)). NGB - North German Basin, PT - Polish Trough, HT - Hamburger Trough.

During the Triassic the conditions began to change towards humid conditions (even though still mainly arid) and thin coal seams can be found in the lowermost Keuper. The extension and rifting caused by the break-up of Pangaea led to salt movement. During the Late Triassic and the Jurassic the depression was flooded again several times, now under humid conditions leading to the deposition of Black 
Shales that are source rocks for the petroleum found in the CEBS. In the Cenozoic continental to deltaic clastics prevail and, forced by the Alpide Orogeny, the salt movement increased again with some salt domes reaching the surface. These salt diapires, pillows and walls are characteristic structures for the CEBS of today. Inbetween them, the thickness of the salt is strongly reduced (fig. 2.3). Due to the high velocities of the salt, these structures pose a big challenge for the processing of seismic data. Frequently occurring anhydrite inclusions in the Zechstein make a monitoring of seismic reflectors and changes in the seismic wavefield from below the Zechstein even more difficult.

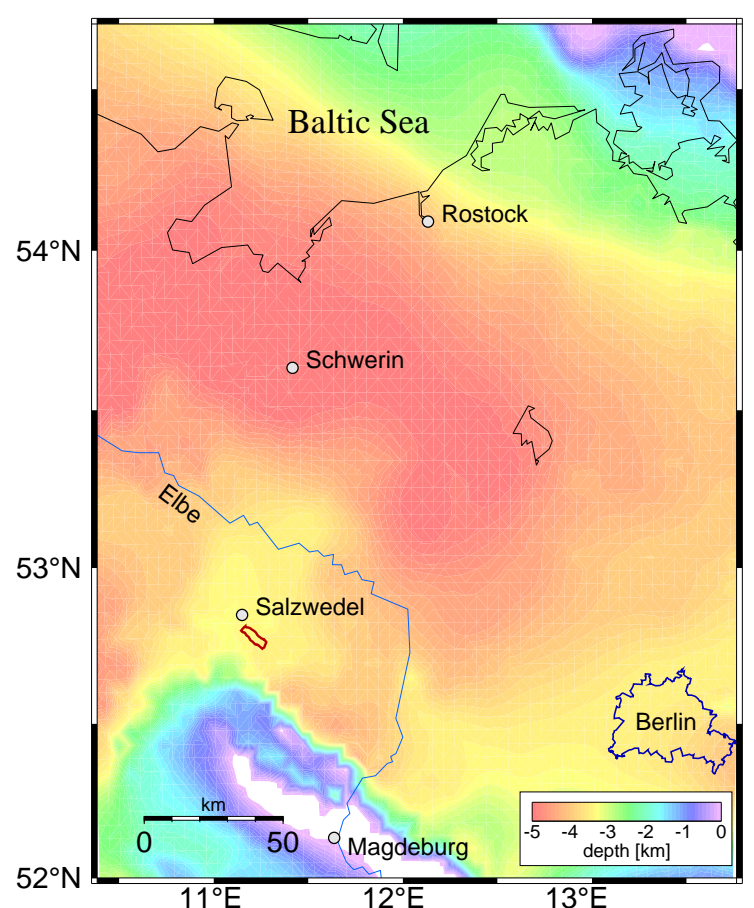

Figure 2.2: The base of the Zechstein. It represents the top of the reservoir (based on a compilation of Scheck-Wenderoth and Lamarche (2005)).

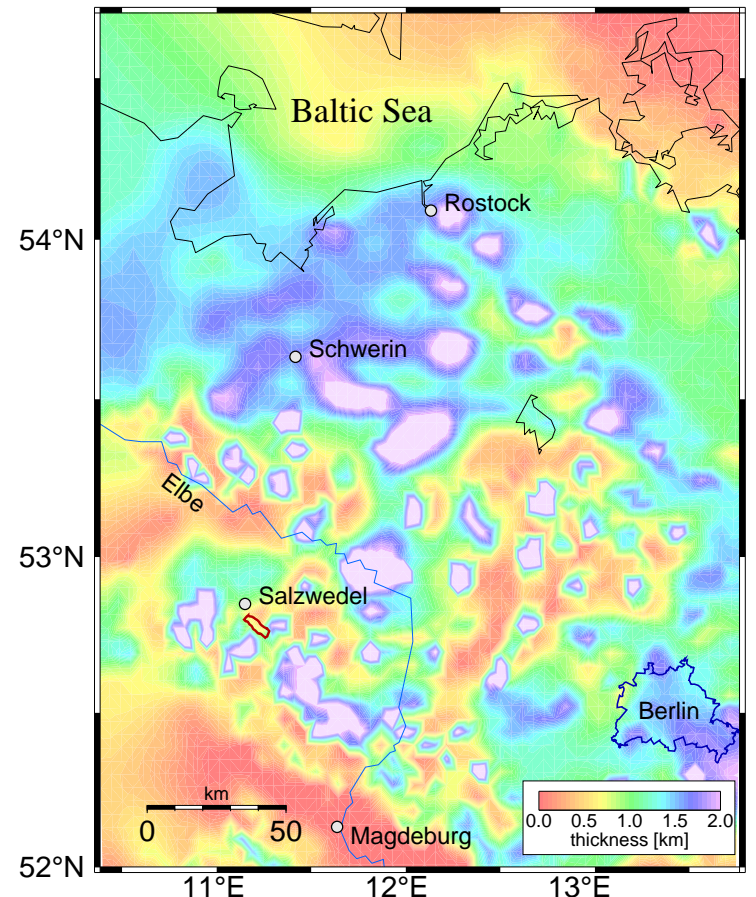

Figure 2.3: The thickness of the Zechstein. The strong variations occur from salt domes and pillows in the NGB (based on a compilation of Scheck-Wenderoth and Lamarche (2005))

\subsubsection{The Altmark gas field}

The study area is the Altmark gas field located within the Southern Permian Basin (SPB, fig. 2.2). With a cumulative gas production of 260 billion $\mathrm{m}^{3}$ it is the largest gas field in Germany and the second largest onshore gas field in Europe (after the Groningen gas field). The peak production level was reached in 1970 and the gas field is now in the state of declining production referred to mature fields. About $70 \%$ of today's gas production originates from this type of gas field category 
(Rückheim et al., 2005). A number of maintenance methods were applied to this gas field (sidetracks, chemical treatments with surfactants and polymers) to exceed the ultimate recovery to over $80 \%$. With a storage potential of about 500 million $t$ of $\mathrm{CO}_{2}$ the Altmark gas field could combine the enhanced gas recovery (EGR) with the sequestration of large amounts of $\mathrm{CO}_{2}$.

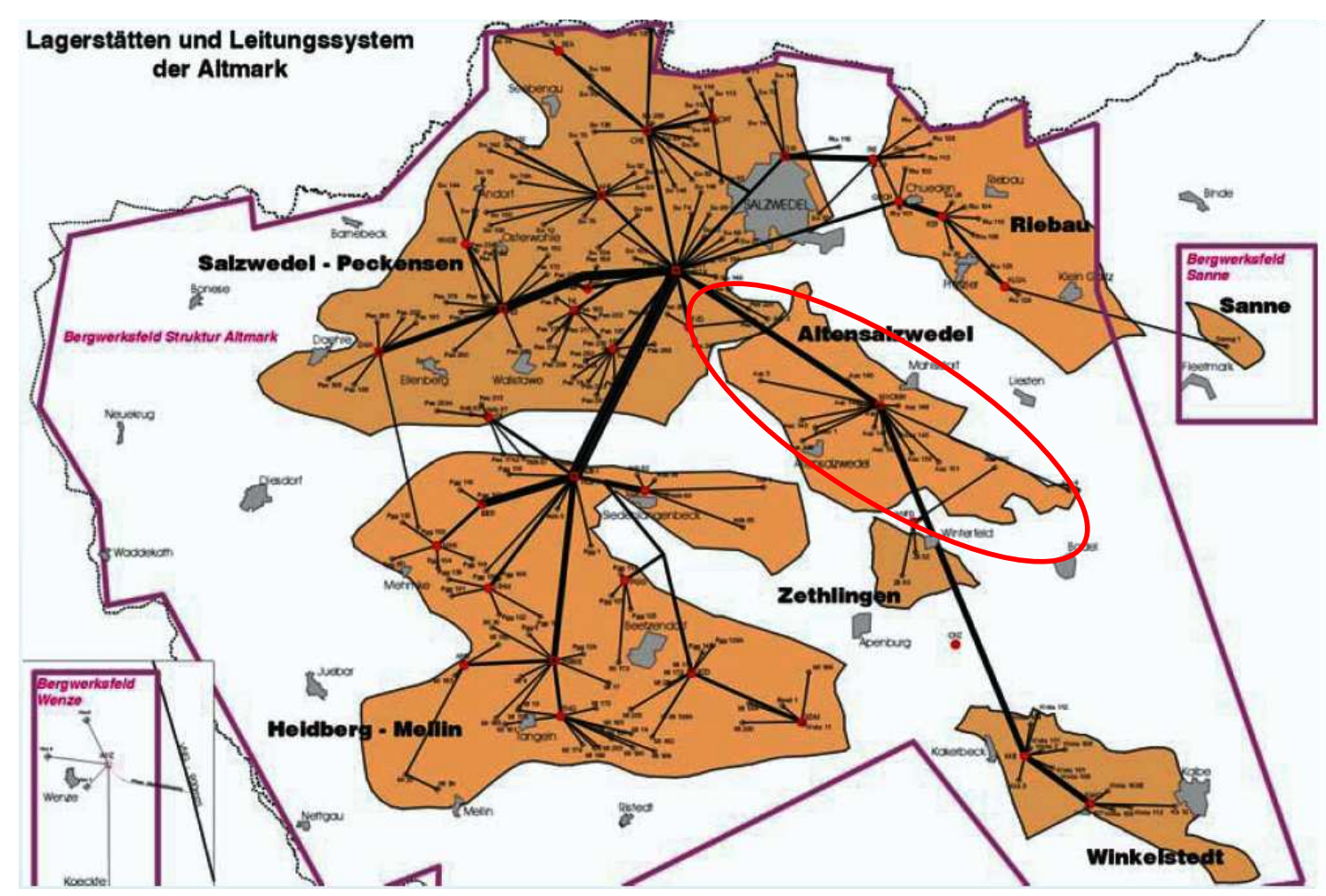

Figure 2.4: Map showing the different blocks of the Altmark gasfield. The project was focused on the Altensalzwedel block (Wendel, 2007).

The Altmark gas field belongs to the major "Rotliegend" trend stretching from the Netherlands (Groningen) to Germany (Altmark). The reservoir is a multilayer sequence of sand- and siltstone separated by shale intercalations. The Zechstein cap rock is highly variable with a minimum thickness of $100 \mathrm{~m}$ up to more than a kilometre due to the presence of salt domes (fig. 2.3). Further sealing formations are salt layers in the Muschelkalk (Triassic) and mudstones in the lower Cretaceous and Cenzoic (Rupelian clay). The gas field is highly compartmentalized and consists of a number of different, hydrodynamically independent blocks.

Within the framework of the CLEAN pilot project an injection of 100,000 $t$ of $\mathrm{CO}_{2}$ into the Altensalzwedel block was planned which is located in the center of the Altmark gas field (fig. 2.4). It is flanked by steep dipping faults. In this part the cap rock (Zechstein) has a medium thickness and no glacial gullies within the Rupelian clay are known because they are partly found in the NEGB. Here the 
reservoir consists of 8 effective layers above the gas-water contact which are in a depth between $3280 \mathrm{~m}$ and $3620 \mathrm{~m}$. Due to the gas production the pore pressure is very low at about $4-5 \mathrm{MPa}$ (initial pore pressure at about $45 \mathrm{MPa}$ ) and the reservoir temperature is $120^{\circ} \mathrm{C}$ to $130^{\circ} \mathrm{C}$. The mean porosity is about $20 \%$.

For the Altmark gas field about 400 exploration and production wells were drilled. However, only two wells are still producing in the Altensalzwedel block (S16 and S14 in figure 2.5). Well S14 was planned as main injector used for the injection of $\mathrm{CO}_{2}$ in the gaseous state while well S17 was an option for injection tests with small amounts of fluid or supercritical $\mathrm{CO}_{2}$ in a later state of the project because pore pressures are higher in the northern part of the reservoir. Well S1 was prepared as observation well for the MSP/VSP experiments.

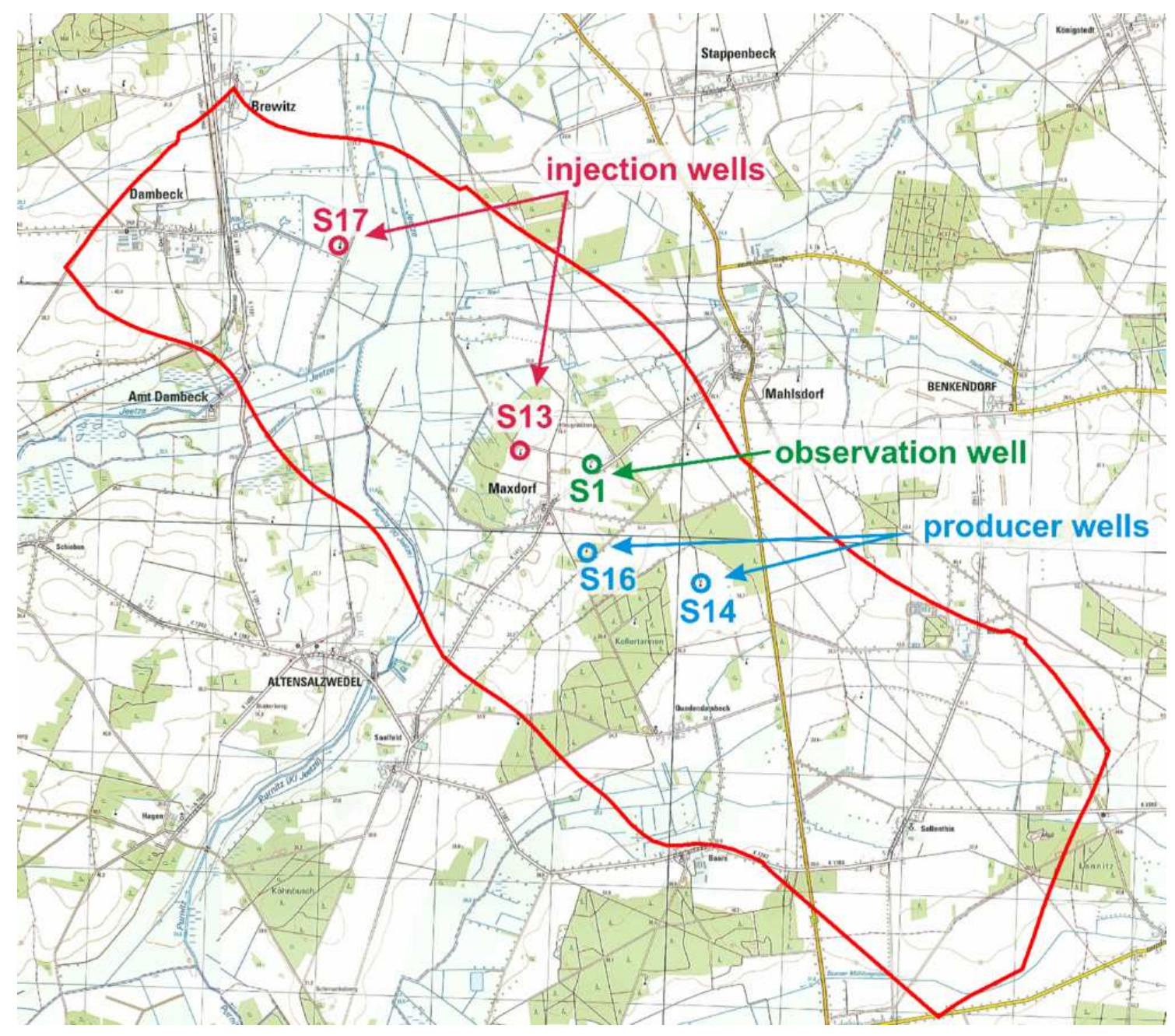

Figure 2.5: The central block of the Altensalzwedel subreservoir with injection wells (red), producers (blue) and the observation well S1 for the seismic monitoring (green). 


\section{$2.2 \mathrm{MSP} / \mathrm{VSP}$ (moving source profiling/ vertical seis- mic profiling)}

For EGR projects, the changes in the seismic wavefield due to $\mathrm{CO}_{2}$ injection will be very small. This leads to the use of seismic borehole experiments with receivers as near as possible at the target zone. MSP/VSP (moving source profiling/ vertical seismic profiling), also known as walkaway VSP, use a vertical arrangement of geophones in a borehole instead of the usual lateral extension of geophones at the surface (fig. 2.6). This geometry allows a closer positioning of the geophones to the target region and, due to shorter travel paths of the seismic waves, less damping of the signal. Most probably, this results in a higher resolution and better signal-tonoise ratio. In VSP experiments upgoing and downgoing waves can be distinguished. Downgoing waves always have a positive vertical velocity component and contain the direct wave as well as twice reflected waves (fig. 2.7). They can be used for a direct measurement of attenuation and geometric divergence and the data can be corrected for (Campbell et al., 2005). They also help to identify and eliminate surface-generated as well as interbed multiples (Hardage, 2000). The downgoing wavefield on the other hand contains the direct reflections and other multiples (fig. 2.7).

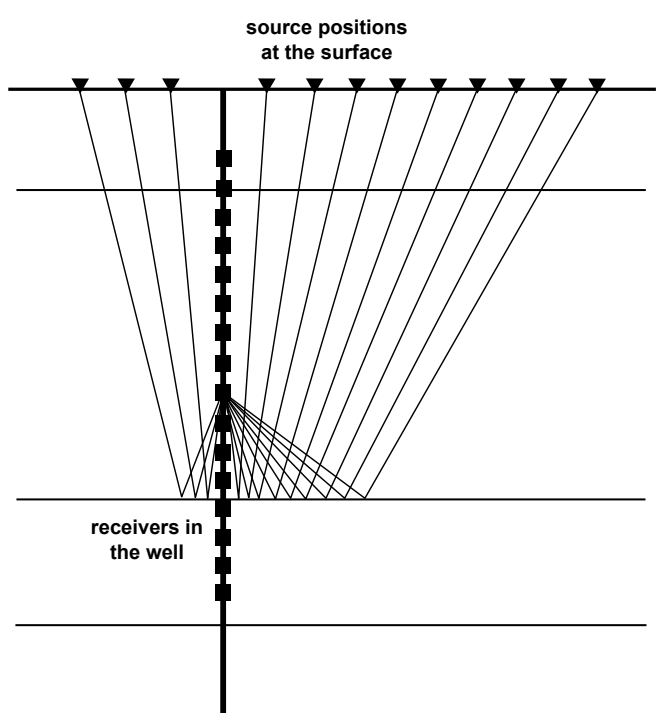

Figure 2.6: Scheme of VSP/MSP experiments. Raypaths are shown for one receiver and different source positions.

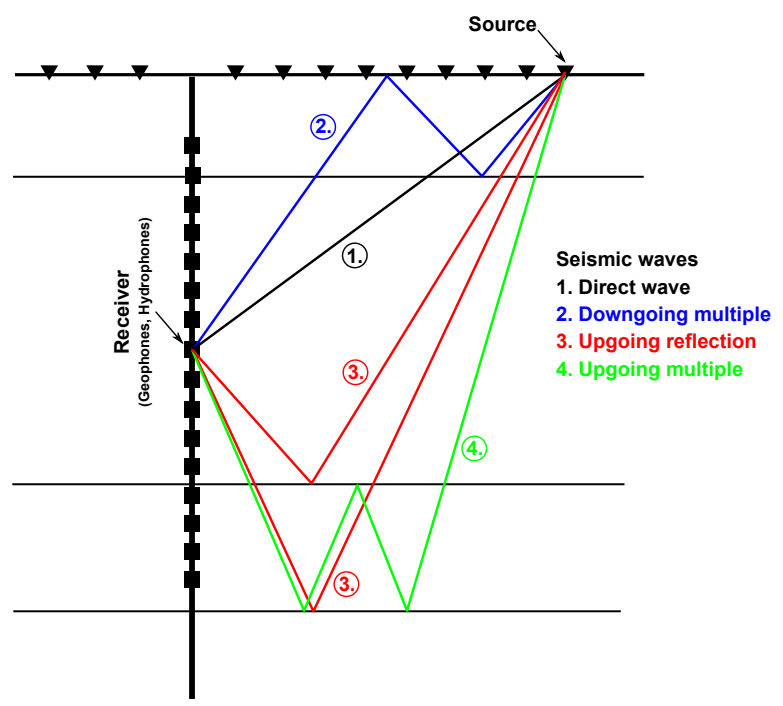

Figure 2.7: Scheme of wave types in VSP experiments.

In principle, two different receivers are used in VSP experiments, hydrophones hanging free in the bore fluid and geophones that are clamped to the wall of the well by a hydraulic extendable arm. 3C-geophones were planned to be used for the 
monitoring in the Altmark as $\mathrm{P}$ - and S-waves, their multiples and potentially the $\mathrm{P} /$ S-ratio can provide information about the injected $\mathrm{CO}_{2}$ in the subsurface.

Combined with sources at increasing offsets from the observation well (MSP), structures in the vicinity of the well can be monitored with high resolution (fig. 2.6). High resolution in all azimuthal directions can be achieved in a 3D VSP survey making use of an areal distribution of surface shotpoints. This kind of a 3D $\mathrm{VSP} / \mathrm{MSP}$ survey will provide the best possible conditions to monitor $\mathrm{CO}_{2}$ injectionrelated changes in the seismic wavefield and was planned for the experiments in the Altmark.

\subsection{Other $\mathrm{CO}_{2}$ sequestration projects}

A large amount of $\mathrm{CO}_{2}$ sequestration projects are carried out worldwide (fig. 2.8). Here we will shortly present some of them that are outstanding and can provide helpful information and experience for the monitoring in the Altmark gas field.

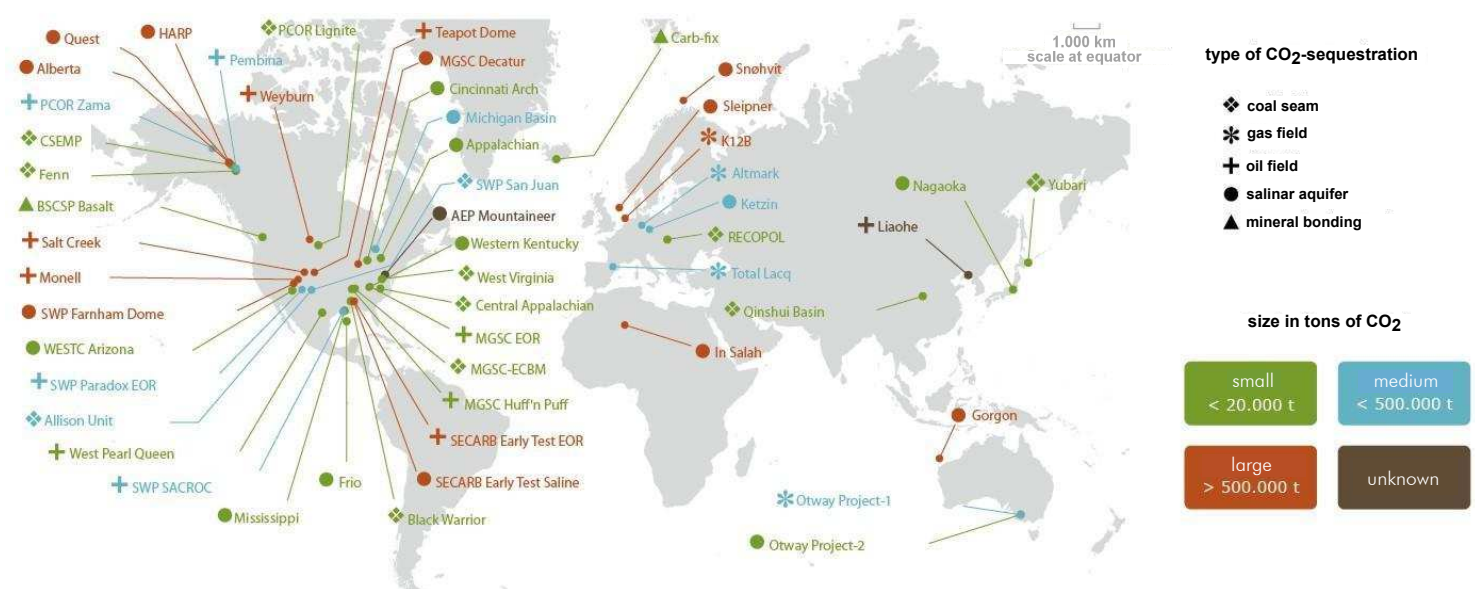

Figure 2.8: $\mathrm{CO}_{2}$ sequestration projects carried out worldwide (edited, CO2CRC, 2010).

\section{Sleipner}

The $\mathrm{CO}_{2}$ sequestration project at the Sleipner gas field in the Norwegian North Sea is the worlds first of industrial scale. It is operated by Statoil and Sleipner partners. Here, the $\mathrm{CO}_{2}$ is separated from the natural gas and is injected back into the Utsira Sand, a saline aquifer formation of late Cenozoic age, $1012 \mathrm{~m}$ below sea level. The injection started in 1996 and more than $11 \mathrm{Mt}$ of $\mathrm{CO}_{2}$ were injected by 2010 (Chadwick et al., 2010). 
The injection is accompanied by a comprehensive monitoring program that is closely linked to the reservoir flow simulations. The key aims of the time-lapse surface seismic program is to track plume migration, demonstrate containment within the storage reservoir, and provide quantitative information as a means to better understand detailed flow processes controlling development of the plume in the reservoir. Therefore a baseline has been carried out in 1994 followed by repeat measurements in 1999, 2001, 2002, 2004, 2006, and 2008.

These experiments imaged the $\mathrm{CO}_{2}$ plume with an extension of about $200 \mathrm{~m}$ in vertical direction (corresponds to the vertical distance from the injection point to the cap rock) and about $3000 \mathrm{~m}$ in horizontal direction in 2008. They show a number of bright subhorizontal reflections within the reservoir indicating $\mathrm{CO}_{2}$ accumulations beneath intrareservoir mudstone intercalations.

A quantitative analysis of the early 1999 data result in a satisfactory match with the flow simulations and a 3D saturation model (Chadwick et al., 2005).

\section{K12-B}

This project is closest to the conditions of the Altmark reservoir. The K12-B field and the Altmark gas field belong to the same reservoir trend. The reservoir is in the Rotliegend about $3800 \mathrm{~m}$ below sea level in the Dutch North Sea and is sealed by a thick Zechstein formation. Like the Altmark gas field it is strongly compartmentalized by sealing faults. The natural gas has a high $\mathrm{CO}_{2}$ content of $13 \%$. This makes the reservoir to be suitable for the long-term storage of $\mathrm{CO}_{2}$. In 2004, 90\% of the initial gas in place was produced. With production the reservoir pressure decreased from initially $40 \mathrm{MPa}$ down to $4 \mathrm{MPa}$. With a reservoir temperature of about $128^{\circ} \mathrm{C}$ the reservoir conditions are nearly identical to the Altmark reservoir. After an injection test in 2004, continuous injection started in 2005 and about 80,000 $t$ of $\mathrm{CO}_{2}$ were injected by 2011. The injected $\mathrm{CO}_{2}$ is separated from the produced natural gas and reinjected into the reservoir (Arts and Vandeveijer, 2011).

The monitoring concept verifies $\mathrm{CO}_{2}$ containment and storage reservoir integrity by $\mathrm{CO}_{2}$ detection methods at the wells and by verifying that the $\mathrm{CO}_{2}$ behaves as expected based on static and dynamic reservoir simulations updated by monitoring data (e.g. pressure, temperature and tracer analysis in the reservoir). No seismic monitoring was carried out at this site.

\section{Otway}

Within the Otway Basin pilot project, of the Australian Cooperative Research Centre for Greenhouse Gas Technologies (CO2CRC), 65, $000 t$ of a $\mathrm{CO}_{2} / \mathrm{CH}_{4}$ mix (ap- 
proximately 80/20) was injected into the depleted Naylor gas reservoir (Waarre C formation) between 2008 and 2009. The gas was produced from a nearby natural accumulation and transported via pipeline. The reservoir is in a depth of about $2 \mathrm{~km}$ and relatively small with $0.5 \mathrm{~km}^{2}$ and an average thickness of $25 \mathrm{~m}$. It is sealed by the Belfast Mudstone.

Due to the expected small impedance changes of 3-5\% that translate into reflectivity changes of about 10\%, an extensive seismic monitoring concept was carried out containing time-lapse 3D surface seismic experiments as well as time-lapse 3D VSP experiments. The baseline for the surface seismic as well as the VSP was carried out in 2007/08 with a weight drop source. The first surface seismic monitoring came in 2009 after about 33, $000 t$ were injected using a vibroseis source. The second repeat of the surface seismic was performed together with the first VSP monitoring in 2010, four months after injection had finished, again using a vibroseis source. Even though different sources were used, the repeatability was excellent with normalized root mean square differences of $20 \%$ in reservoir depth for the 3D surface seismic and of $10 \%$ for the 3D VSP. The primary task of the surface seismic was an assurance monitoring. It showed that no significant amount of $\mathrm{CO}_{2}$ escaped from the reservoir into overlain formations (e.g. saline Paaratte aquifer), while the VSP with its higher repeatability showed some anomaly between the injection and monitoring well in the difference sections (Urosevic et al., 2011).

In a second phase, which is actually in planning phase, a small amount of about 10, $000 t$ of $\mathrm{CO}_{2}$ will be injected into the overlaying aquifer sequence simulating a leakage scenario and determining the sensitivity of the monitoring survey to a $\mathrm{CO}_{2}$ leakage.

\section{Ketzin}

The Ketzin pilot site, located near Berlin, Germany, and led by the GFZ German Research Centre for Geosciences, is Europe's longest-operating on-shore $\mathrm{CO}_{2}$-storage site. The project is of research scale with an amount of $53.000 \mathrm{t}$ of $\mathrm{CO}_{2}$ injected into a saline aquifer in a 630 - $650 \mathrm{~m}$ deep anticlinal sandstone structure. The injection that started in 2008 is accompanied by an extensive monitoring program integrating geological, geophysical and geochemical investigations. Based on these results static geological modelling and dynamic simulations are regularly updated (Martens et al., 2012).

The geophysical monitoring comprises a geoelectric monitoring, a borehole monitoring (pressure-temperature measurements) and a seismic monitoring. Different seismic methods have been employed such as time-lapse Vertical-Seismic Profiling (VSP), Crosswell, Moving Source Profiling (MSP) and surface seismics. The base- 
line was carried out in fall 2005, the first repeat in September 2009 and the second repeat in February 2011.

The seismic experiments were able to show $\mathrm{CO}_{2}$-induced changes in the amplitude of the seismic wavefield and to monitor the propagation of the $\mathrm{CO}_{2}$. The experiments with a "star" geometry showed good agreement with the 3D-data and that this approach can be successfully used to monitor the migration of the $\mathrm{CO}_{2}$ within the reservoir. However, these tests also showed the necessity of a regular source and receiver pattern and fold distribution (Ivandic et al., 2012).

\section{In-Salah}

A joint industry project was set up to monitor the $\mathrm{CO}_{2}$ storage process into the aquifer leg of the gas producing Krechba field in central Algeria. It is operated by BP together with its joint venture partner Sonatrach and Statoil that joined when injection started in 2004. It was planned to inject $17 \mathrm{Mt}$ of $\mathrm{CO}_{2}$ over a period of 5 years for long-term storage ( $3 M t$ by 2009). This amount is removed from the production stream of several gas fields with a $\mathrm{CO}_{2}$ content of $5-10 \%$. The reservoir is a $20 \mathrm{~m}$ thick sandstone layer from which the Krechba field produces $\mathrm{CO}_{2}$ rich gas. The injection depth is in about $1850 \mathrm{~m}$ to $1950 \mathrm{~m}$. The reservoir is sealed by a $950 \mathrm{~m}$ thick sequences of mixed Carboniferous mudstones (Mathieson et al., 2010).

The injection is accompanied by a variety of geochemical (soil gas monitoring, saline and shallow aquifer sampling), geophysical and production techniques. A detailed picture of the $\mathrm{CO}_{2}$ plume was built from reservoir modelling, history matching of the $\mathrm{CO}_{2}$ breakthrough, pressure data and satellite deformation data from satellite-based interferometric synthetic aperture radar (InSAR), the most valuable monitoring method. The pressure data also indicate, that the $\mathrm{CO}_{2}$ is sealed in the formation and not leaking off.

A preinjection 3D seismic survey was acquired in 1997, focused on imaging the reservoir section and not the overburden. The $3 \mathrm{D}$ seismic survey was completed in 2010 and has to be compare with the baseline survey. Results are not known until now.

\section{Weyburn}

For enhanced oil recovery (EOR), the injection of $\mathrm{CO}_{2}$ into the Weyburn field in southeastern Saskachewan, Canada, operated by EnCana started in October 2000 together with a multidisciplinary International Energy Agency Greenhouse Gas (IEA GHG) $\mathrm{CO}_{2}$ Monitoring and Storage Project (CO2MSP). The field was discovered in 1954 and it was flooded with water since 1960 to increase the pore pressure (Ma and 
Morozov, 2010). Crude oil is produced from the Midale beds of the Mississippian Charles Formation in about $1450 \mathrm{~m}$ depth. The reservoir has a thickness of $16 \mathrm{~m}$ to $28 \mathrm{~m}$ and contains two litho-stratigraphic units: the lower Vuggy Limestone and the upper Marly Dolostone. It is sealed by anhydritic dolostones and anhydrites. The cumulative $\mathrm{CO}_{2}$ injection surpassed $12 \mathrm{Mt}$ in 2008. The $\mathrm{CO}_{2}$ is captured from the Dakota Gasification Plant in Beulah, North Dakota, liquefied and transported over $320 \mathrm{~km}$ to the injection site via pipeline (Wilson and Monea, 2004).

A large amount of 81 subtasks formed the research program attending the injection, which are categorized into four major themes. One of them was the monitoring concept that comprised a variety of seismic and geochemical sampling methods. In this framework passive and active seismic surveys were acquired with a $3 \mathrm{D}$ seismic baseline measured in December 1999 prior to injection. The repeats in 2001, 2002, 2004, and 2007 demonstrated the ability to monitor physical changes in the Weyburn reservoir induced by $\mathrm{CO}_{2}$ injection. The results proved high sensitivity to the presence of low $\mathrm{CO}_{2}$ saturations of 5-10 molar \%. They also matched very well with rock and fluid property measurements, logbased synthetic seismic modelling, and reservoir simulation/production history (White, 2009).

The passive seismic monitoring showed a correlation of micro seimicity rates with periods of elevated $\mathrm{CO}_{2}$ injection rates and also with changes in production activities in nearby wells. The distribution of injection-related event locations also appeared to correlate with the regions of $\mathrm{CO}_{2}$ saturation that have been identified using 4D seismic. However, overall the rates of seismicity were low indicating no significant geomechanical deformation of the reservoir (Verdon et al., 2010).

\section{Conclusion}

The K12B project in the Netherlands has no seismic monitoring program, presumably because the expected changes are too small. However, conclusions about the spatial and temporal evolution of the $\mathrm{CO}_{2}$ and a possible leakage can only be given from breakthrough times at wells and pressure information combined with reservoir simulations. The Otway project on the contrary applied a full seismic monitoring with combined walkaway VSP and surface seismic. However, the results of the VSP show slight changes within the reservoir region but the conditions were also slightly better than those for the Altmark reservoir (shallower depth, less strong reflecting overlaying formations). The the Sleipner and Ketzin project showed that a monitoring of $\mathrm{CO}_{2}$ displacing water in industrial as well as in research scale produces much larger changes and so leakages of a certain amount of $\mathrm{CO}_{2}$ into water bearing layers in the Altmark might be visible in reflection seismic data. 



\section{Chapter 3}

\section{Modelling of the seismic wavefield}

In order to establish a seismic monitoring concept accompanying a $\mathrm{CO}_{2}$-EGR project it is of high interest estimating the changes in the seismic wavefield that are generated by the injection of $\mathrm{CO}_{2}$. Therefore the seismic wavefield has to be modeled in a forward modeling approach, where the wave equations have to be solved. But since no exact analytical solution exist for most subsurface structures, various techniques were developed among which finite-difference (FD) approaches gained in importance with increasing computer power in the last decades. We use the program fdveps_mpi by Bohlen (2008) that is based on a two-dimensional finite-difference (2D FD) approach. Attenuation and dispersion is introduced to this program by application of the model of the "generalized standard linear solid" (GSLS) (Emmerich and Korn, 1987). The derivations are calculated on a standard staggered grid (SSG) (Robertsson et al., 1994).

In the first part of this chapter we will give a rough overview of viscoelastic 2D FD theory with regard to the used program. Afterwards we explain the optional parameters and the accommodations and models we used. Finally the preprocessing steps applied to the output seismograms are outlined.

\subsection{Theory}

For simplification the theory of viscoelasticity is explained for the $1 \mathrm{D}$ case for simple shear in an isotropic and homogeneous material. Afterwards it is extended to the case of P-SV wave propagation in a 2D linear viscoelastic medium. The resulting system of partial differential equations is discretized in a SSG FD approach.

Here we will make use of the Einstein summation convention and express derivatives as $\partial_{i}\left(\partial_{i}=\frac{\partial}{\partial i}\right)$. The dot on a symbol indicates its time derivative $\left(\dot{u}=\partial_{t} u=\right.$ $\left.\frac{\partial u}{\partial t}\right)$. 


\subsubsection{Viscoelasticity}

Viscoelasticity in general can be described as a combination of elastic and viscous elements. In mechanical models the elastic element is represented by a spring with the spring constant $k$ and the viscous element is described by a dashpot with viscosity $\eta$. They relate the stress $\sigma$ to the strain $\epsilon$ in the following way

$$
\sigma=k \epsilon
$$

and

$$
\sigma=\eta \dot{\epsilon} .
$$

These two mechanical models can be combined either in series or in parallel. The combination of a spring and a dashpot in parallel is called the Kelvin-Voigt model and represents the viscoelasticity of a solid. The Maxwell model is a combination of a spring and a dashpot in series, and it essentially represents a fluid. When combining a Maxwell body with a spring in parallel it is called the standard linear solid (fig. 3.1). It represents a viscoelastic solid (Blanch et al., 1993) and its relaxation function is given by

$$
\Psi(t)=k_{0}+k_{1} e^{-\frac{t}{\tau_{\sigma}}}
$$

with

$$
\tau_{\sigma}=\frac{\eta_{1}}{k_{1}}
$$

This can be extended to an arbitrary linear viscoelastic material by connecting several standard linear solids in parallel

$$
\Psi(t)=\sum_{i=1}^{L} k_{0 i}+\sum_{l=1}^{L} k_{l} e^{-\frac{t}{\tau_{\sigma l}}}
$$

with the stress relaxation time

$$
\tau_{\sigma l}=\frac{\eta_{l}}{k_{l}} .
$$


This is equivalent to a spring in parallel with $L$ Maxwell bodies, which becomes obvious when inserting

$$
K_{0}=\sum_{i=1}^{L} k_{0 i}
$$

and is called the model of the "generalized standard linear solid" (GSLS) (Fig. 3.1). With the strain retardation time

$$
\tau_{\epsilon l}=\frac{\eta_{l}}{K_{0}}+\frac{\eta_{l}}{k_{l}}
$$

one yields a representation for the relaxation function of an arbitrary viscoelastic material of the form

$$
\Psi(t)=K_{0}\left[1-\sum_{l=1}^{L}\left(1-\frac{\tau_{\epsilon l}}{\tau_{\sigma l}}\right) e^{-\frac{t}{\tau_{\sigma l}}}\right] .
$$

In a $1 \mathrm{D}$ viscoelastic model for a simple shear in an isotropic homogeneous material, the stress-strain relation is given by

$$
\sigma=[\Psi(t) H(t)] * \dot{\epsilon}=\partial_{t}[\Psi(t) H(t)] * \epsilon
$$

where $*$ indicates convolution, $\Psi$ is the relaxations function of a GSLS and $\mathrm{H}(\mathrm{t})$ is the Heaviside function (Blanch et al., 1993). The stress relaxation function $\Psi(t) H(t)$ is the viscoelastic analog to the Lamé constant $\mu$ in linear elasticity.

The complex modulus $M_{C}(\omega)$ of the material is defined as the Fourier transform of the time derivation of the stress relaxation function

$$
M_{C}(\omega)=\mathbf{F}\left\{\partial_{t}[\Psi(t) H(t)]\right\} .
$$

When inserting equation 3.9 and calculating the differentiation and integration (Fourier-Transformation) of 3.11 this leads to

$$
M_{C}(\omega)=K_{0}\left(1-L+\sum_{l=1}^{L} \frac{1+i \omega \tau_{\epsilon l}}{1+i \omega \tau_{\sigma l}}\right) .
$$

In the elastic limit, which is reached when the dashpot is eliminated $\left(\eta_{l} \rightarrow 0\right)$ and consequently $\tau_{\epsilon l} \rightarrow 0$ and $\tau_{\sigma l} \rightarrow 0$ and which is equivalent with the low frequency limit $(\omega \rightarrow 0)$, the complex modulus goes to the relaxed modulus $K_{0}$, which coincides with the elastic modulus (Carcione et al., 1988).

Now the seismic quality factor $\mathrm{Q}$ can be calculated, which describes the attenuation properties of rocks, with

$$
Q=\frac{\boldsymbol{R e}\left\{M_{C}(\omega)\right\}}{\boldsymbol{I m}\left\{M_{C}(\omega)\right\}},
$$


where $\boldsymbol{R} \boldsymbol{e}$ and $\boldsymbol{I} \boldsymbol{m}$ represent the real- and imaginary part of the complex modulus, respectively. Substituting 3.12 into 3.13 this gives

$$
Q\left(\omega, \tau_{\sigma l}, \tau\right)=\frac{1+\sum_{l=1}^{L} \frac{\omega^{2} \tau_{\sigma l}^{2}}{1+\omega^{2} \tau_{\sigma l}^{2}} \tau}{\sum_{l=1}^{L} \frac{\omega \tau_{\sigma l}}{1+\omega^{2} \tau_{\sigma l}^{2}} \tau}
$$

where

$$
\tau=\frac{\tau_{\epsilon l}}{\tau_{\sigma l}}-1
$$

It is a general assumption that the quality factor $\mathrm{Q}$ is constant or only slowly varying within the seismic frequency range, which is shown in a number of experiments (Spencer, 1981; Murphy, 1982). In viscoelastic theory this can be achieved by determining the optimization parameters $\tau_{\sigma l}$ and $\tau$ of a limited number of Maxwell bodies in a least-squares inversion by minimizing

$$
J\left(\tau_{\sigma l}, \tau\right)=\int_{\omega_{1}}^{\omega_{2}}\left(Q^{-1}\left(\omega, \tau_{\sigma l}, \tau\right)-\tilde{Q}^{-1}\right)^{2} d \omega
$$

where $\tilde{Q}$ is the desired constant quality factor.

With the complex modulus we can also define a complex velocity $v_{C}(\omega)$

$$
v_{C}(\omega)=\sqrt{\frac{M_{C}(\omega)}{\rho}},
$$

where $\rho$ is the density. This description of viscoelasticity explains not only attenuation, but also dispersion of seismic waves propagating through the earth (Liu et al., 1976).

\subsubsection{Viscoelastic wave equations}

Now this approach can be used to calculate the propagation of seismic P-SV waves in a $2 \mathrm{D}$ homogeneous, isotropic, linear viscoelastic medium. We start from the constitutive equation of P-SV waves in a linear elastic 2D medium

$$
\sigma_{i j}=\lambda \delta_{i j} \epsilon_{k k}+\mu \epsilon_{i j}
$$

with $\mathrm{i}, \mathrm{j}, \mathrm{k}=\mathrm{x}, \mathrm{z} . \sigma_{i j}$ are the components of the stress tensor, $\epsilon_{i j}$ the components of the strain tensor and $\lambda$ and $\mu$ are the elastic Lamé constants. In the viscoelastic case, this equation becomes (Blanch et al., 1995)

$$
\sigma_{i j}=\dot{\Lambda} * \delta_{i j} \epsilon_{k k}+2 \dot{M} * \epsilon_{i j}
$$


Taking the time derivation of both sides and defining

$$
\Pi=\Lambda+2 M
$$

we get

$$
\dot{\sigma}_{i j}=(\dot{\Pi}-2 \dot{M}) * \delta_{i j} \dot{\epsilon}_{k k}+2 \dot{M} * \dot{\epsilon}_{i j}
$$

With this definition of $\Pi$ in equation 3.20 attenuation can be treated separately for $\mathrm{P}$ - and S-waves and the quality factor $Q^{p}$ and $Q^{s}$ can be defined for P- and S-waves, respectively, by following the procedure described in equations 3.11 to 3.13 . $\Pi$ and $M$ are the stress relaxation functions and are defined as it was derived in equation 3.10

$$
\Pi(t)=\pi\left[1-\sum_{l=1}^{L}\left(1-\frac{\tau_{\epsilon l}^{p}}{\tau_{\sigma l}}\right) e^{-\frac{t}{\tau_{\sigma l}}}\right] H(t)
$$

and

$$
M(t)=\mu\left[1-\sum_{l=1}^{L}\left(1-\frac{\tau_{\epsilon l}^{s}}{\tau_{\sigma l}}\right) e^{-\frac{t}{\tau_{\sigma l}}}\right] H(t),
$$

where $\pi=\lambda+2 \mu$ and $\tau_{\epsilon l}^{p}$ and $\tau_{\epsilon l}^{s}$ are the strain retardation times for the $l$ th relaxation mechanism for $\mathrm{P}$ - and S-waves, respectively. The moduli $\pi$ and $\mu$ are defined by $v_{p}(\omega)$ and $v_{s}(\omega)$, which are the P-and S-wave velocity at the center frequency of the source signal $\omega_{0}$, defined as the real part of the complex phase velocity from equation 3.17 (Carcione et al., 1988; Bohlen, 2002)

$$
\pi=v_{p}^{2}\left(\omega_{0}\right) \rho \boldsymbol{R} e\left\{\sqrt{\frac{1}{1+\sum_{l=1}^{L} \frac{i \omega_{0} \tau_{\sigma l}}{1+i \omega_{0} \tau_{\sigma l}} \tau^{p}}}\right\}
$$

and

$$
\mu=v_{s}^{2}\left(\omega_{0}\right) \rho \boldsymbol{R} e\left\{\sqrt{\frac{1}{1+\sum_{l=1}^{L} \frac{i \omega_{0} \tau_{\sigma l}}{1+i \omega_{0} \tau_{\sigma l}} \tau^{s}}}\right\} .
$$

Computing the convolutions of equation 3.19 and replacing the strain with

$$
\dot{\epsilon}_{i j}=\frac{1}{2}\left(\partial_{i} v_{j}+\partial_{j} v_{i}\right)
$$

yields

$$
\dot{\sigma}_{i j}=\left[\pi\left(1+L \tau^{p}\right)-2 \mu\left(1+L \tau^{s}\right)\right] \partial_{k} v_{k}+\mu\left(1+L \tau^{s}\right)\left(\partial_{i} v_{j}+\partial_{j} v_{i}\right)+\sum_{i=1}^{L} r_{i j l}
$$


where $v_{i}$ is the particle velocity and $r_{i j l}$ is the memory function. The memory equations

$$
\dot{r}_{i j l}=-\frac{1}{\tau_{\sigma l}}\left(\left(\pi \tau^{p}-2 \mu \tau^{s}\right) \partial_{k} v_{k}+\mu \tau^{s}\left(\partial_{i} v_{j}+\partial_{j} v_{i}\right)+r_{i j l}\right)
$$

and the momentum equation

$$
\rho \dot{v}_{i}=\partial_{j} \sigma_{i j}+f_{i}
$$

complete the system of first-order coupled partial differential equations that describe the propagation of seismic waves in a $2 \mathrm{D}$ viscoelastic medium (or $3 \mathrm{D}$ viscoelastic medium, if $\mathrm{i}, \mathrm{j}, \mathrm{k}=\mathrm{x}, \mathrm{y}, \mathrm{z}) . f_{i}$ are body forces. They can be neglected for normal seismic wavelength and outside of the source region. Equations 3.27, 3.28 and 3.29 have to be solved to calculate the seismic wavefield at any time and at any location in the model.

\subsubsection{Finite-difference method}

A number of different techniques exist to solve equations 3.27 - 3.29 and to simulate the seismic wavefield. Among them finitedifference techniques proved to work very efficiently even in models of high complexity. With increasing computer power their application increased.

Compared to the centered finitedifference approach, the standard staggeredgrid (SSG) finite-difference scheme has the advantage that its error is 4 times smaller (Aki and Richards, 1980). In this method the stresses and velocities are defined on different grids that are shifted by half a

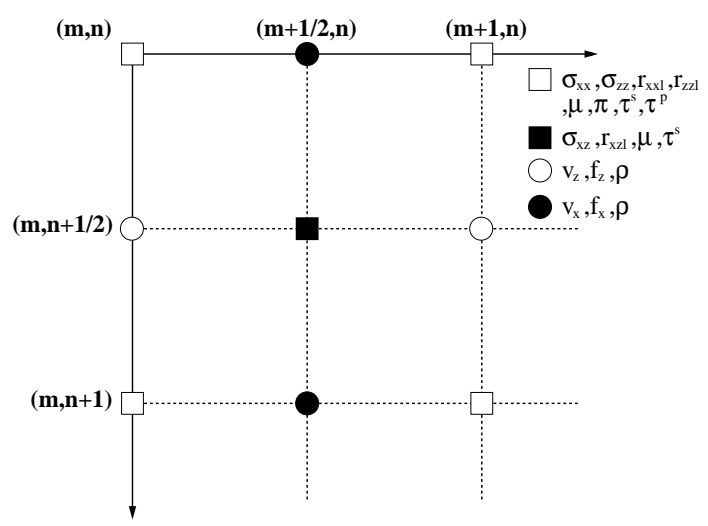

Figure 3.2: Location of dynamic variables $\left(\sigma_{x x}, \sigma_{x z}, \sigma_{z z}, r_{x x l}, r_{x z l}, r_{z z l}, v_{x}, v_{z}, f_{x}\right.$ and $\left.f_{z}\right)$ and material parameters $\left(\mu, \pi, \tau^{s}, \tau^{p}\right.$ and $\rho)$ in a staggered finite difference grid. grid point (fig. 3.2) (Virieux, 1986; Levander, 1988). The SSG method was first extended to viscoelastic wave propagation by Robertsson et al. (1994).

In an SSG approach the equations describing the propagation of P-SV waves in 
$2 \mathrm{D}$ can be formulated as follows

$$
\begin{aligned}
\sigma_{x x}^{k^{+}}(m, n)= & \sigma_{x x}^{k^{-}}(m, n)+\pi(m, n) \triangle t\left(1+L \tau^{p}(m, n)\right)\left(D_{x}^{-} v_{x}^{k}\left(m^{+}, n\right)+D_{z}^{-} v_{z}^{k}\left(m, n^{+}\right)\right) \\
& -2 \mu(m, n) \triangle t\left(1+L \tau^{s}(m, n)\right) D_{z}^{-} v_{z}^{k}\left(m, n^{+}\right) \\
& +\frac{\triangle t}{2} \sum_{l=1}^{L}\left(r_{x x l}^{k^{+}}(m, n)+r_{x x l}^{k^{-}}(m, n)\right)
\end{aligned}
$$

$$
\begin{aligned}
\sigma_{x z}^{k^{+}}\left(m^{+}, n^{+}\right)= & \sigma_{x z}^{k^{-}}\left(m^{+}, n^{+}\right) \\
& +2 \mu\left(m^{+}, n^{+}\right) \triangle t\left(1+L \tau^{s}\left(m^{+}, n^{+}\right)\right)\left(D_{z}^{+} v_{x}^{k}\left(m^{+}, n\right)+D_{x}^{-} v_{z}^{k}\left(m, n^{+}\right)\right) \\
& +\frac{\triangle t}{2} \sum_{l=1}^{L}\left(r_{x z l}^{k^{+}}\left(m^{+}, n^{+}\right)+r_{x z l}^{k^{-}}\left(m^{+}, n^{+}\right)\right)
\end{aligned}
$$

$$
\begin{aligned}
\sigma_{z z}^{k^{+}}(m, n)= & \sigma_{z z}^{k^{-}}(m, n)+\pi(m, n) \triangle t\left(1+L \tau^{p}(m, n)\right)\left(D_{x}^{-} v_{x}^{k}\left(m^{+}, n\right)+D_{z}^{-} v_{z}^{k}\left(m, n^{+}\right)\right) \\
& -2 \mu(m, n) \triangle t\left(1+L \tau^{s}(m, n)\right) D_{x}^{-} v_{x}^{k}\left(m^{+}, n\right) \\
& +\frac{\triangle t}{2} \sum_{l=1}^{L}\left(r_{z z l}^{k^{+}}(m, n)+r_{z z l}^{k^{-}}(m, n)\right),
\end{aligned}
$$$$
r_{x x l}^{k^{+}}(m, n)=\left(1+\frac{\triangle t}{2 \tau_{\sigma l}}\right)^{-1}\left[\left(1-\frac{\triangle t}{2 \tau_{\sigma l}}\right) r_{x x l}^{k^{-}}(m, n)\right.
$$

$$
\begin{aligned}
& -\frac{\pi(m, n) \triangle t}{\tau_{\sigma l}} \tau^{p}(m, n)\left(D_{x}^{-} v_{x}^{k}\left(m^{+}, n\right)+D_{z}^{-} v_{z}^{k}\left(m, n^{+}\right)\right) \\
& \left.+\frac{2 \mu(m, n) \triangle t}{\tau_{\sigma l}} \tau^{s}(m, n) D_{z}^{-} v_{z}^{k}\left(m, n^{+}\right)\right]
\end{aligned}
$$$$
\begin{aligned}
r_{x z l}^{k^{+}}\left(m^{+}, n^{+}\right)= & \left(1+\frac{\triangle t}{2 \tau_{\sigma l}}\right)^{-1}\left[\left(1-\frac{\triangle t}{2 \tau_{\sigma l}}\right) r_{x z l}^{k^{-}}\left(m^{+}, n^{+}\right)\right. \\
& \left.-\frac{\mu\left(m^{+}, n^{+}\right) \triangle t}{\tau_{\sigma l}} \tau^{s}\left(m^{+}, n^{+}\right)\left(D_{z}^{+} v_{x}^{k}\left(m^{+}, n\right)+D_{x}^{+} v_{z}^{k}\left(m, n^{+}\right)\right)\right]
\end{aligned}
$$

$$
\begin{aligned}
r_{z z l}^{k^{-}}(m, n)= & \left(1+\frac{\triangle t}{2 \tau_{\sigma l}}\right)^{-1}\left[\left(1-\frac{\triangle t}{2 \tau_{\sigma l}}\right) r_{z z l}^{k^{-}}(m, n)\right. \\
& -\frac{\pi(m, n) \triangle t}{\tau_{\sigma l}} \tau^{p}(m, n)\left(D_{x}^{-} v_{x}^{k}\left(m^{+}, n\right)+D_{z}^{-} v_{z}^{k}\left(m, n^{+}\right)\right) \\
& \left.+\frac{2 \mu(m, n) \triangle t}{\tau_{\sigma l}} \tau^{s}(m, n) D_{x}^{-} v_{x}^{k}\left(m^{+}, n\right)\right]
\end{aligned}
$$




$$
\begin{aligned}
v_{x}^{k}\left(m^{+}, n\right)= & v_{x}^{k-1}\left(m^{+}, n\right)+\frac{\triangle t}{\rho\left(m^{+}, n\right)}\left[D_{x}^{+} \sigma_{x x}^{k^{-}}(m, n)+D_{z}^{-} \sigma_{x z}^{k^{-}}\left(m^{+}, n^{+}\right)\right] \\
& +f_{x}^{k}\left(m^{+}, n\right) \\
v_{z}^{k}\left(m, n^{+}\right)= & v_{z}^{k-1}\left(m, n^{+}\right)+\frac{\triangle t}{\rho\left(m, n^{+}\right)}\left[D_{x}^{-} \sigma_{x z}^{k^{-}}\left(m^{+}, n^{+}\right)+D_{z}^{+} \sigma_{z z}^{k^{-}}(m, n)\right] \\
& +f_{z}^{k}\left(m, n^{+}\right) .
\end{aligned}
$$

They are discretized on an equidistant grid, where $h$ is the grid size in both spatial directions $(h=\triangle x=\Delta z)$ and $\triangle t$ is the time interval. The derivatives in time are appriximated by use of a Crank-Nicholson scheme (Bohlen, 2002)

$$
\left.\partial_{t} f(x, z, t)\right|_{k} \approx \frac{f^{k^{+}}(m, n)-f^{k^{-}}(m, n)}{\triangle t} .
$$

$D_{i}^{+}$and $D_{i}^{-}$are the forward and backward operator used to calculate the spatial derivatives, which are e.g.

$$
\left.\partial_{i} f(i)\right|_{m^{+}} \approx D_{i}^{+} f(m)=\frac{1}{h}[f(m+1)-f(m)]
$$

and

$$
\left.\partial_{i} f(i)\right|_{m^{-}} \approx D_{i}^{-} f(m)=\frac{1}{h}[f(m)-f(m-1)]
$$

for accuracy of second order and

$$
\left.\partial_{i} f(i)\right|_{m^{+}} \approx D_{i}^{+} f(m)=\frac{1}{24 h}[-f(m+2)+27(f(m+1)-f(m))+f(m-1)]
$$

and

$$
\left.\partial_{i} f(i)\right|_{m^{-}} \approx D_{i}^{-} f(m)=\frac{1}{24 h}[-f(m+1)+27(f(m)-f(m-1))+f(m-2)]
$$

for accuracy of fourth order and correspondingly for higher orders, with i, $\mathrm{j}=\mathrm{x}, \mathrm{z}$. $m$ and $n$ are the indices of the two spatial directions $\mathrm{x}$ and $\mathrm{z}$ and $\mathrm{k}$ is the index for time. The superscripts indicate $m^{+}=(m+1 / 2) h$ and $m^{-}=(m-1 / 2) h$.

But time and space intervals cannot be selected completely arbitrarily. They have to fulfil the following criteria to yield stable results. In order to avoid numerical dispersion, which can be easily misinterpreted as physical dispersion, a certain number of grid points per wavelength is needed. This is achieved when the criterion

$$
h \leq \frac{v_{s_{\min }}}{2 f_{c} N}
$$


is satisfied, where $v_{s_{\min }}$ is the minimum S-wave velocity, $f_{c}$ is the center frequency of the source wavelet and $\mathrm{N}$ is the number of grid points per minimum wavelength and depends on the order of the FD algorithm.

The time steps have to satisfy the stability criterion

$$
\triangle t \leq c \frac{h}{v_{p_{\max }} \sqrt{D}} .
$$

It depends on the grid spacing $\mathrm{h}$, the maximum $\mathrm{P}$-wave velocity $v_{p_{\max }}$, the dimension $\mathrm{D}$ of the model ( $\mathrm{D}=2$ in our $2 \mathrm{D}$-case) and a factor $\mathrm{c}$, that depends on the order of spatial accuracy. If it is not satisfied, the wave equation is no longer honoured and the wavefield will "explode".

\subsection{The program fdveps_mpi and modeling pa- rameters}

The program fdveps_mpi calculates the seismic wavefield at any time step and at each subsurface point in the model on basis of equations $3.30-3.37$. The following explains the options of the program, the parameters that have to be set in the parameter file and the accommodations that were selected for the following simulations.

\subsubsection{FD-order}

The time derivative is always calculated with the Crank-Nicholson scheme and of second order accuracy, but the accuracy in space can be selected between second and twelfth order (changing the forward and backward operator in equations 3.39 and 3.40). In all following simulations an accuracy in space of eighth order $(\mathrm{O}(2,8))$ was used.

\subsubsection{Parallelization}

The program is parallelized with the free Message Passing Interface (MPI), that allows to run computational intensive simulation on clusters of workstations. The user has to compartmentalize the model grid by defining the number of processors in horizontal and vertical direction in the parameter file.

\subsubsection{Model}

Besides the time interval, which we set to $\triangle t=0.0001 \mathrm{~s}$, also the model dimension and the distance between grid points have to be set. We used a model of $9000 \mathrm{~m}$ 
horizontal and $4950 \mathrm{~m}$ vertical extension by a grid size of $1 \mathrm{~m}$. The models have to be equidistant. The model parameters, on which the wavefield is calculated, are read in from files that are generated with an external program. These are density, $\mathrm{P}$-wave and $\mathrm{S}$-wave velocity. Though it is possible to estimate the elastic as well as the viscoelastic wavefield, $Q_{P}$ and $Q_{S}$ are optional parameters and are read in from separate files only when simulating the viscoelastic wavefield.

To simulate the seismic wavefield a horizontally layered subsurface structure of the Altmark was used (fig. 3.3), which is eligible, as the depth of the top of the reservoir layers varies by only about $25 \mathrm{~m}$ throughout the central Altensalzwedel reservoir block and also the sediment layers within the North German Basin are known to approximate a $1 \mathrm{D}$ stratification in absence of salt domes, which is valid for the Altensalzwedel region (fig. 2.3). The density information of the subsurface were taken from well log data and core sample information from the observation well S1. Their depth ranges were adapted to the much finer P-wave velocity information, which were taken from existing VSP-data from the observation well. They comprise first arrival times from the surface to receivers in $34 \mathrm{~m}$ to $3550 \mathrm{~m}$ depth (approx. every $50 \mathrm{~m}$ in the upper part of the well and every $25 \mathrm{~m}$ in the reservoir region). A low velocity weathering layer was implemented and the model was extended to approximately $5000 \mathrm{~m}$ depth by taking velocity layers of $50 \mathrm{~m}$ thickness with a randomly around $4500 \mathrm{~m} / \mathrm{s}$ varying $\mathrm{P}$-wave velocity. This was done to implement additional reflectors, because lots of reflections from below the reservoir are necessary for the use of the analysis methods (especially for coda wave interferometry).

Because not enough information about the S-wave velocity exist,the subsurface was assumed to consist of a Poisson material and the S-wave velocity was calculated by $v_{s}=v_{p} / \sqrt{3}$.

Finally the reservoir layer was implemented. Therefore the velocities and density were determined (see chapter 7) and inserted in a layer with a thickness of $24 \mathrm{~m}$ at a depth of $3425 \mathrm{~m}$, the cumulative thickness and the mean depth of the eight reservoir layers in the Altensalzwedel block that were the target horizons for the $\mathrm{CO}_{2}$ injection. This approximation is feasible, as the wave length of the source signal in this depth is more than $100 \mathrm{~m}$ and the thin single reservoir layers will not be resolved separately. In this layer also velocity and density changes that are generated when $\mathrm{CO}_{2}$ is injected, are implemented around a defined injection point (fig. 3.3, circled). 

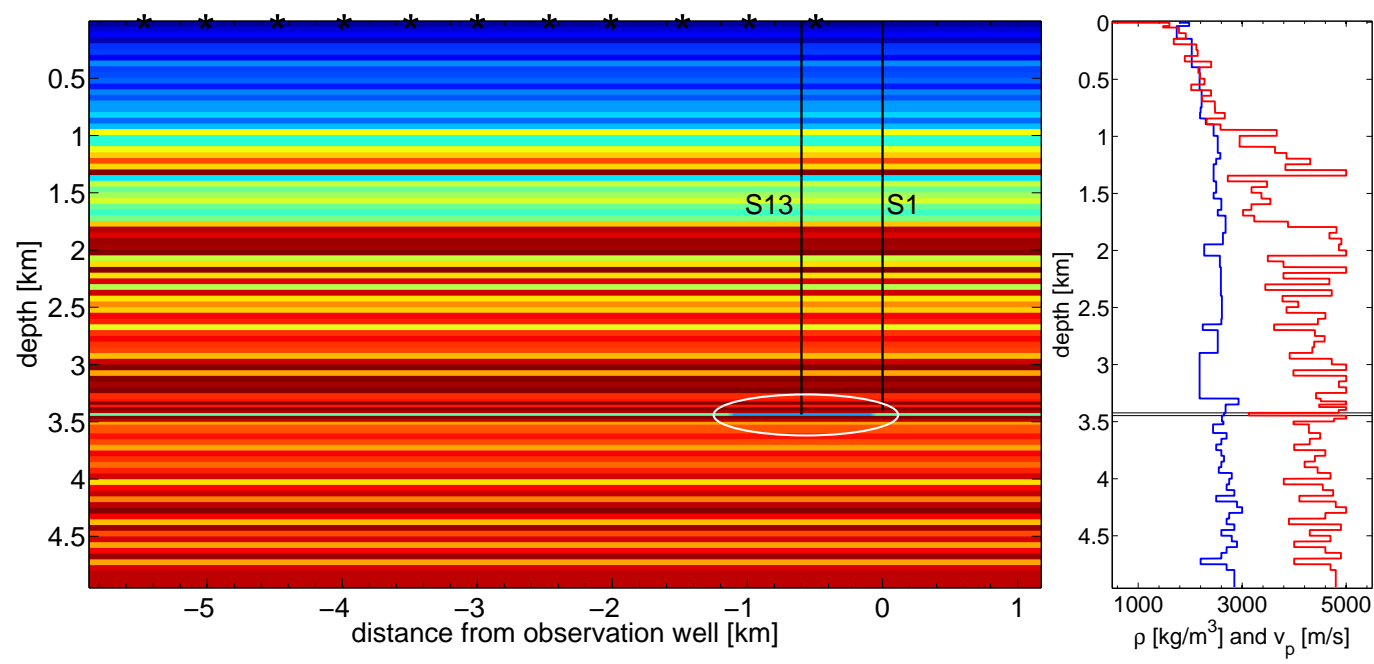

Figure 3.3: Left: Model of $\mathrm{P}$-wave velocity of the Altensalzwedel reservoir region. S1 indicates the observation well, S13 the injection well and the stars at the surface are source positions. The reservoir layer is in about $3425 \mathrm{~m}$ and the $\mathrm{CO}_{2}$ injection is simulated by changing the rock properties in a certain range around the injection well (circled). Right: Density (blue) and P-wave velocity profile (red).

\subsubsection{Attenuation}

It is possible to calculate viscoelastic wave propagation by setting the number of relaxation mechanisms $L$ in the parameter file to a value greater than zero. Otherwise for $L=0$ the elastic wavefield is simulated. Additionally the relaxation frequencies $f_{l}=2 \pi / \tau_{\sigma l}$ and $\tau$ has to be set to describe the frequency dependence of the quality factor (equation 3.14). In this case the attenuation is homogeneous and equal for Pand S-waves. A separate MATLAB-program can be used to find best values for $L, f_{l}$ and $\tau$ in order to fit an arbitrary constant $Q$-function $(Q(\omega)=$ const $)$ with a leastsquares approach. To achieve spatial distributed $Q$-factors, $Q_{p^{-}}$and $Q_{s}$-model files can be read in. Then the $Q$-functions are assumed to be constant for all frequencies.

The following models were calculated in the elastic case as no information about damping factors for the Altmark region was acquirable.

\subsubsection{Boundaries}

At the top of the model a stress-free surface is applied while the remaining three edges are absorbing boundaries. The width of the damping zone and the attenuation at the edges in percent can be selected in order to achieve as less reflections from the model edges as possible. From a number of tests it was found that a zone of a thickness of 50 grid points $(50 \mathrm{~m})$ and a damping with an amplitude decay of $8 \%$ at 
the edges gives reasonable good results.

\subsubsection{Source}

The user can distinguish between three build in source functions or define an own. The build in wavelets are a Ricker, a Fuchs-Müller and a $\sin ^{3}$. Also the source type is optional (explosive and point source in $\mathrm{x}$ - and z-direction). In the case of the directive forces, the source is introduced into the model by assigning the body force term $f_{i}$ with the source amplitude in equation 3.37 and 3.36 (for a vertical or horizontal point source, respectively). For all models in this study a Ricker wavelet excited by a point source in z-direction was used, which excites $\mathrm{P}$ - as well as S-waves and is similar to the wavefield of a vibroseis source. The center frequency was set to $25 \mathrm{~Hz}$ with a maximum frequency of about $50 \mathrm{~Hz}$. The source position is read from a separate source file and was always set to the surface just with varying offsets.

\subsubsection{Receiver}

Receiver lines can be placed in the model easily. Therefore only the position of the first and the last receiver and the inter receiver distance have to be set in the parameter file. To simulate a VSP experiment the receivers were placed in a vertical line with a distance of 10 grid points reaching from the surface down to $3900 \mathrm{~m}$. They were placed at an offset of $600 \mathrm{~m}$ from the injection point, which is the distance between the injection well S13 and the observation well S1 (fig. 3.3). For an arbitrary distribution of receivers the locations can also be read in from a separate receiver file. The seismogram output is also variable. Output of particle velocities, curl and divergence (P-and S-wavefield, respectively) and of the pressure field (hydrophones) is possible. Sample rates can be whole-numbers of the time steps. The program was chosen to give out the horizontal and vertical velocities as well as the curl and divergence of the velocity field with a sample rate of $0.2 \mathrm{~ms}$, which is a bit less than the minimum sample rate of the VSP-tools available for the experiments $\left(\triangle t_{\min }=0.25 \mathrm{~ms}\right)$. The output was in Seismic Unix (SU) format.

\subsection{Preprocessing}

Even though the output data are perfect, means noise free, a few preprocessing steps were needed to prepare the data. These are a time shift to correct for a delay in the source signal and the separation of the up-going wavefield from the entire wavefield for enhanced examination. 


\subsubsection{Static shift}

The maximum amplitude (displacement) of a Ricker wavelet is always excited at one and a half periods $\left(1.5 / f_{c}\right)$ by the programm (fig. 3.4). So in a first step we shifted the seismic traces by a constant factor of $60 \mathrm{~ms}(1.5 / 25 \mathrm{~Hz})$ in order to set the maximum amplitude excitation to zero time (zero-phasing). This is necessary especially for the migration tests described in chapter 5 , where reflectors would be shifted to greater depth if not corrected.

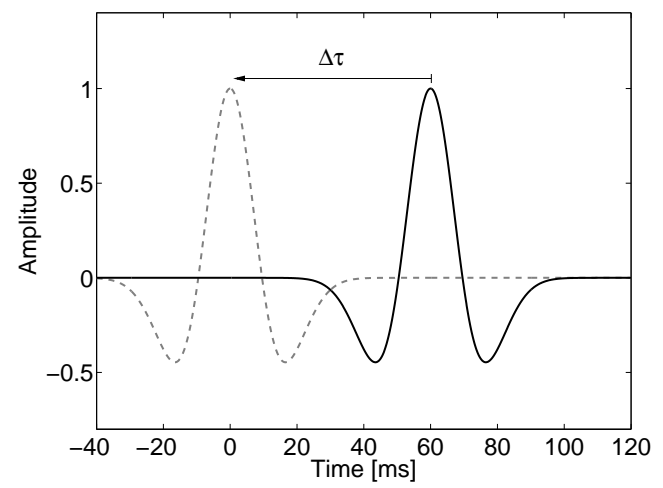

Figure 3.4: The maximum amplitude is shifted by one and a half periods and has to be shifted back to zero time for migration.

\subsubsection{Wavefield separation}

Afterwards the up-going wavefield was separated from the complete wavefield by use of a frequency-wavenumber (f-k) filter. This is standard in vertical seismic profiling. The up-going wavefield contains all waves that were reflected below the receiver position and consequently contains all direct reflections, while the downgoing wavefield contains the direct waves. Both parts contain multiples, but the up-going wavefield contains statistically more multiples that travelled the reservoir region at least ones. This is important in coda-wave interferometry.

For separating the wavefields the complete wavefield is transformed from timespace ( $\mathrm{t}-\mathrm{x}$ ) domain to the $\mathrm{f}-\mathrm{k}$ domain by 2D Fourier transformation. Though Fourier transformation expects periodicity and to suppress artefacts in the Fourier transformed function arising from the abrupt end of the traces, the edges were damped in time and space. The wavefield in frequency-wavenumber $(\mathrm{f}-\mathrm{k})$ domain ranges from $-f_{\text {Nyquist }} / 2$ to $f_{\text {Nyquist }} / 2$ and from $-k_{\text {Nyquist }} / 2$ to $k_{\text {Nyquist }} / 2$. The limits are given by

$$
f_{\text {Nyquist }}=\frac{\pi}{\triangle t} \quad \text { and } \quad k_{N y q u i s t}=\frac{\pi}{\triangle z},
$$


where $\Delta t$ is the sample rate and $\Delta z$ is the inter receiver spacing (Suprajitno and Greenhalgh, 1985). Due to periodicity, the first and third quadrant are identical as well as the second and fourth quadrant.

As the z-axis is defined to be positive to depth, down-going waves will have positive and up-going waves will have negative velocities. From the dispersion relation $(v=\omega / k)$ the down-going wavefield can be identified as the one in the first (and third) quadrant and the up-going wavefield as the one in the second (and fourth) quadrant (fig. 3.5). So the filter function damps the even quadrants and in the back transformed seismic section only the upgoing wavefield remains.

The effectiveness of the filter is shown in figure 3.6 for a simple 2D two-layer velocity model (fig. 3.6 e). The seismic sec-

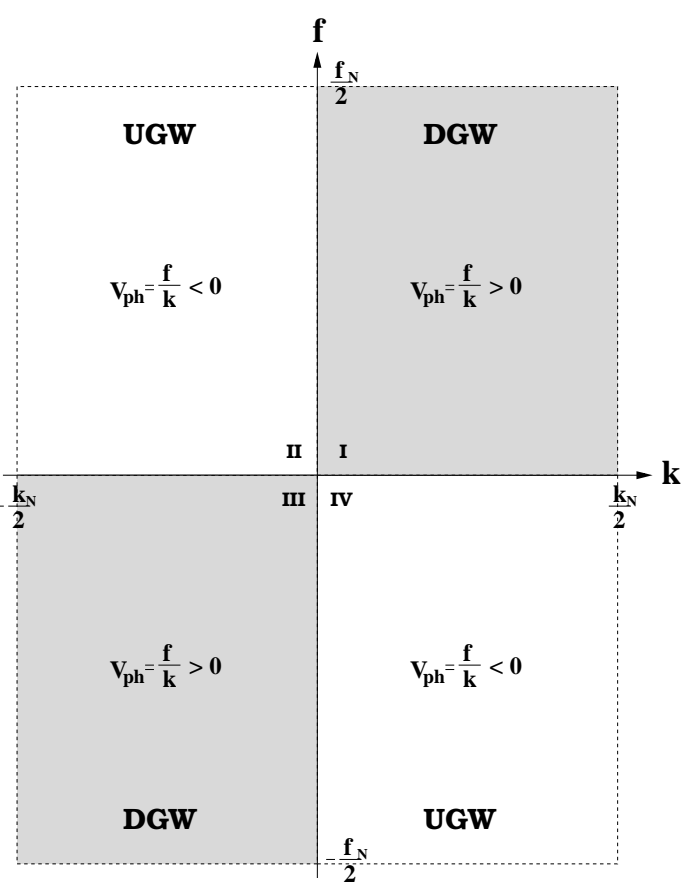

Figure 3.5: Classification in fk-domain. Quadrant I and III have positive phase velocities and contain DGW, quadrants II and IV UGW with negative phase velocities. $k_{N}$ is the Nyquist wavenumber and $f_{N}$ the Nyquist frequency. tions before (a) and after filtering (c) show P-waves, b) and d) are showing the first and second quadrant of the $\mathrm{f}-\mathrm{k}$ spectra of the sections above. It can be seen that the filter suppresses the downgoing wavefield very effectively and nearly only the up-going wavefield remains. Artefacts in terms of smearing outs appearing at the onsets of up-going waves are minimized by a filter function that is Gaussian-damped at the edges, but cannot be avoided completely as it can be seen at the onset of the SP-converted wave after about $2 \mathrm{~s}$ (fig. $3.6 \mathrm{~d}$ ).

Figure 3.7 shows the filter applied to the more complicated Altensalzwedelmodel. Comparison of a) and c) suggests that the filter works very well also in this case. The missing of information in the upper $300 \mathrm{~m}$ and the lower $100 \mathrm{~m}$ result from the damping of the edges in the $\mathrm{x}$ - $\mathrm{t}$ domain before the Fourier transformation. Here the strongest artefacts occur, which is not disturbing as the upper and very deep part is of minor interest.

These steps were applied to all calculated seismograms and further analysis was implemented on the preprocessed data. 

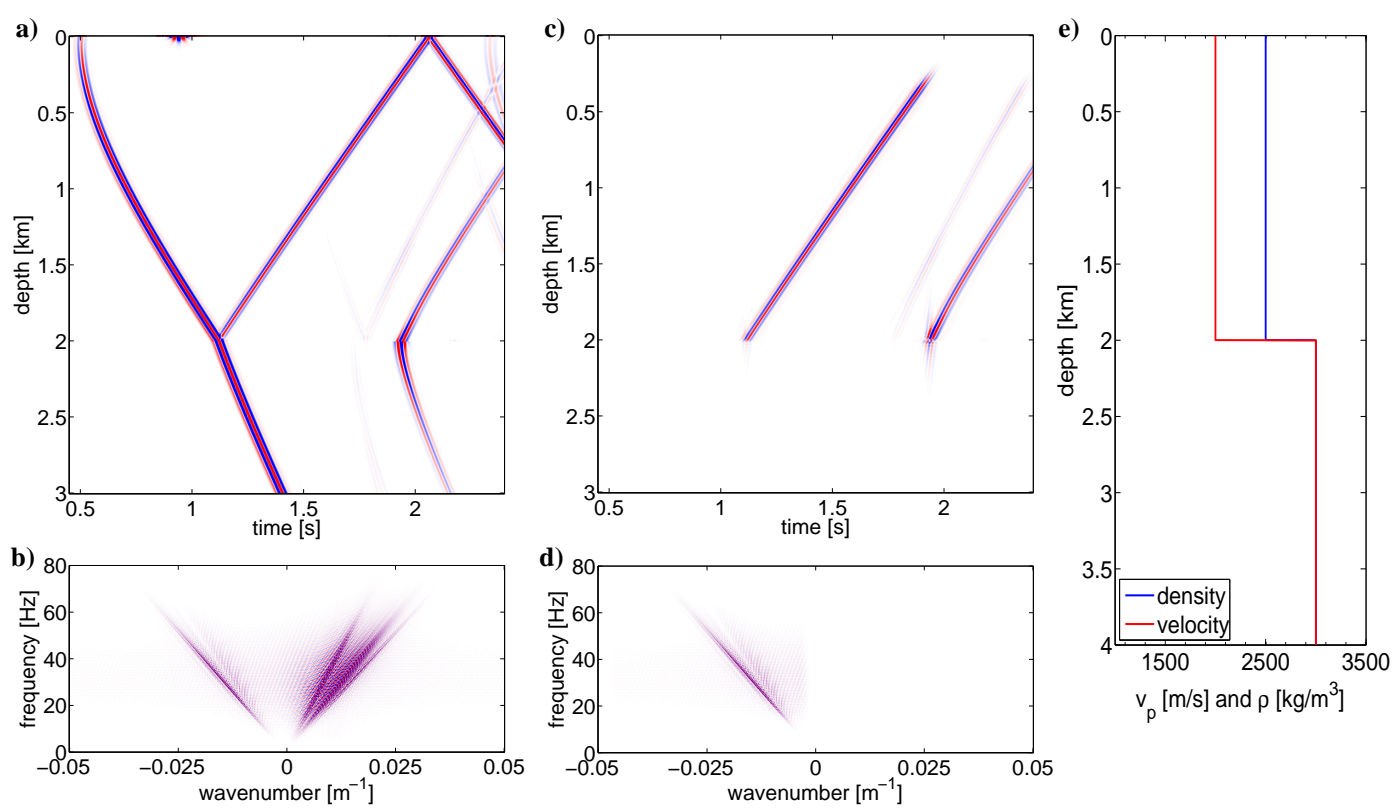

Figure 3.6: P-wavefield (a) in a two-layer model (e) and the corresponding f-k spectrum (b). Upgoing waves (c) of the wavefield in (a) by damping the right part (1. quadrant) in the f-k-spectrum (d).
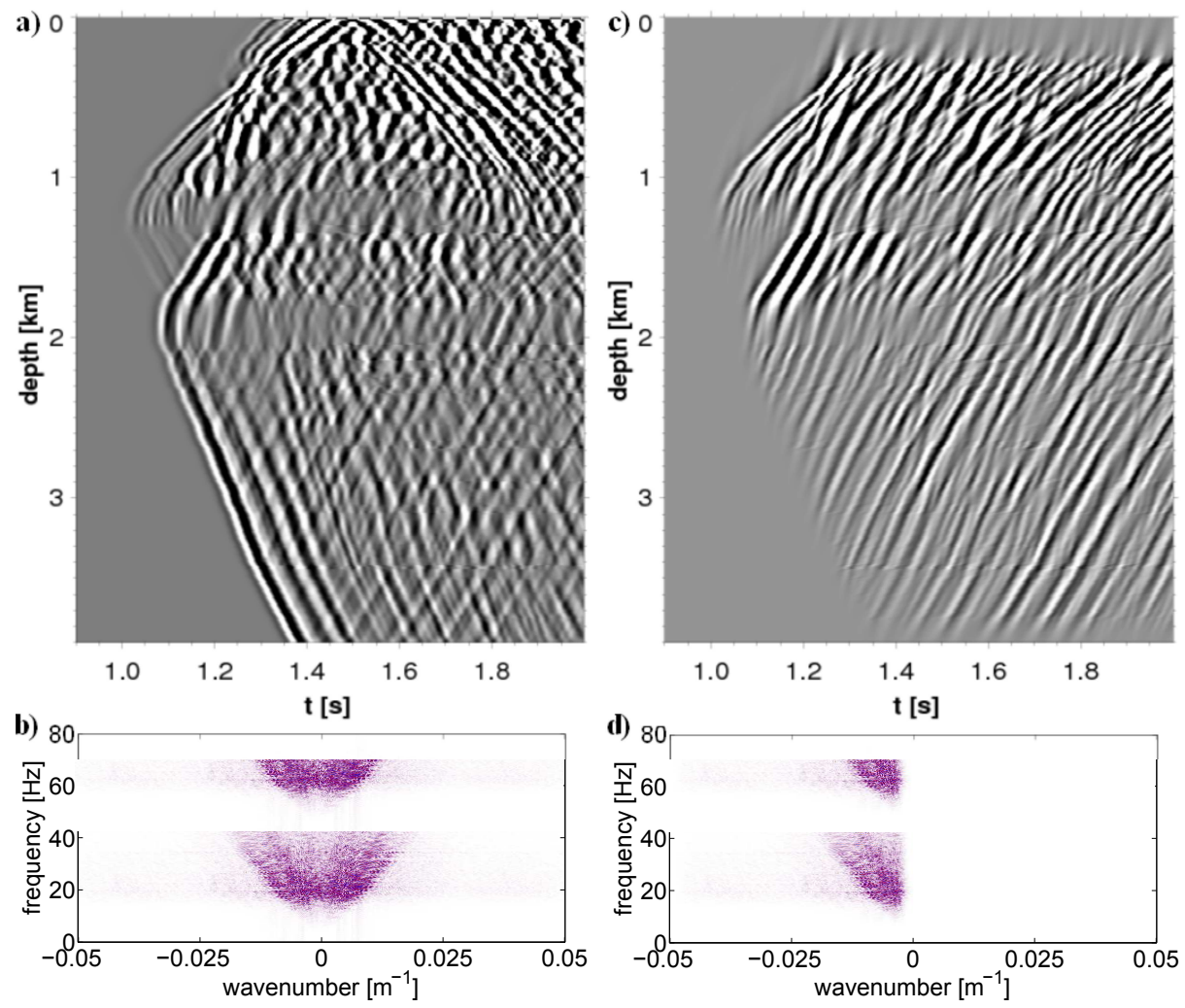

Figure 3.7: P-wavefield calculated on the Altensalzwedel-model (a), the separated up-going wavefield (c) and their corresponding f-k spectra (b and d). 

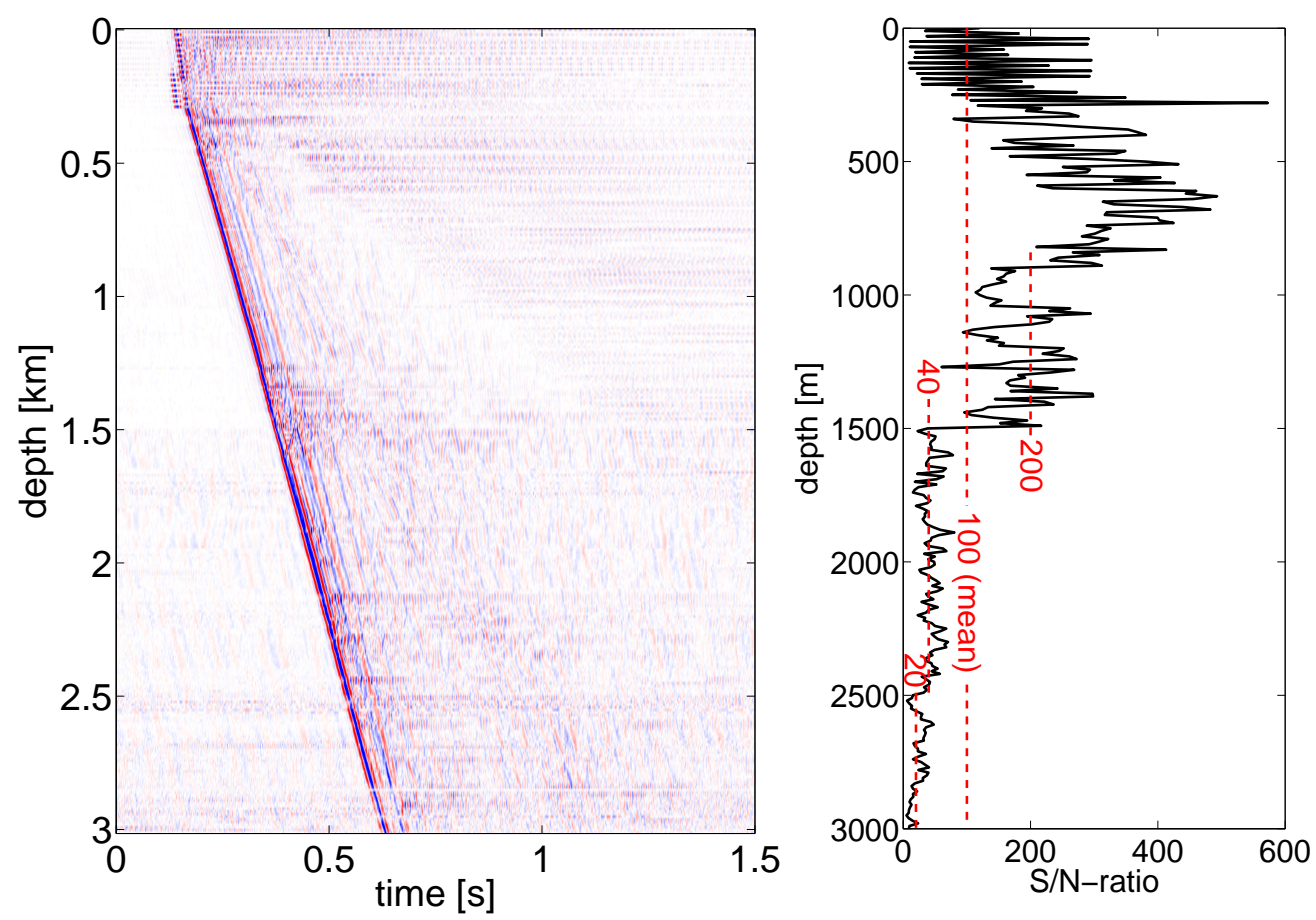

Figure 3.8: Measured data and corresponding S/N-ratio.

\subsubsection{Noise}

In field experiments one will never have ideal conditions. Data will always be contaminated by noise. Even though the noise level in VSP experiments is usually better than for surface seismics, uncorrelated as well as correlated noise occur. In order to analyze the stability of the used methods under imperfect conditions, data were contaminated with artificial white noise (random noise with equal power spectrum).

The Signal-to-Noise $(\mathrm{S} / \mathrm{N})$ ratio was defined as the ratio of the root-mean-squares (rms) of the signal and of the noise. The rms of the signal was calculated in a time window of 2.5 periods around the first break of each trace and the noise-rms in the first $0.8 \mathrm{~s}$

$$
S N R=\frac{\frac{1}{2.5 T} \int_{t=t_{f b}-0.5 T}^{t_{f b}+2 T} u^{2}(t) d t}{\frac{1}{20 T} \int_{t=0}^{20 T} u^{2}(t) d t},
$$

where $\mathrm{T}$ is the period length at centre frequency, $t_{f b}$ is the first break time and $\mathrm{u}(\mathrm{t})$ is the seismic wavefield.

The different $\mathrm{S} / \mathrm{N}$ ratios that were tested, were oriented at data that were recorded in April 2010 at the KTB (continental deep drilling site) as a test run prior to field experiments. They were recorded in the pilot hole in a VSP geometry 
(source offset just a few tens of meters) with Mini-Vibe source (DMT). The receivers were 17 three-component (3C) SlimWave ${ }^{T M}$ geophones from Sercel. Starting from about $3000 \mathrm{~m}$ depth the chain was moved up in $150 \mathrm{~m}$ steps with an overlap of two receivers, respectively. At each step the shot was repeated 5 times and stacked. The data example and the corresponding $\mathrm{S} / \mathrm{N}$-ratio is shown in figure 3.8.

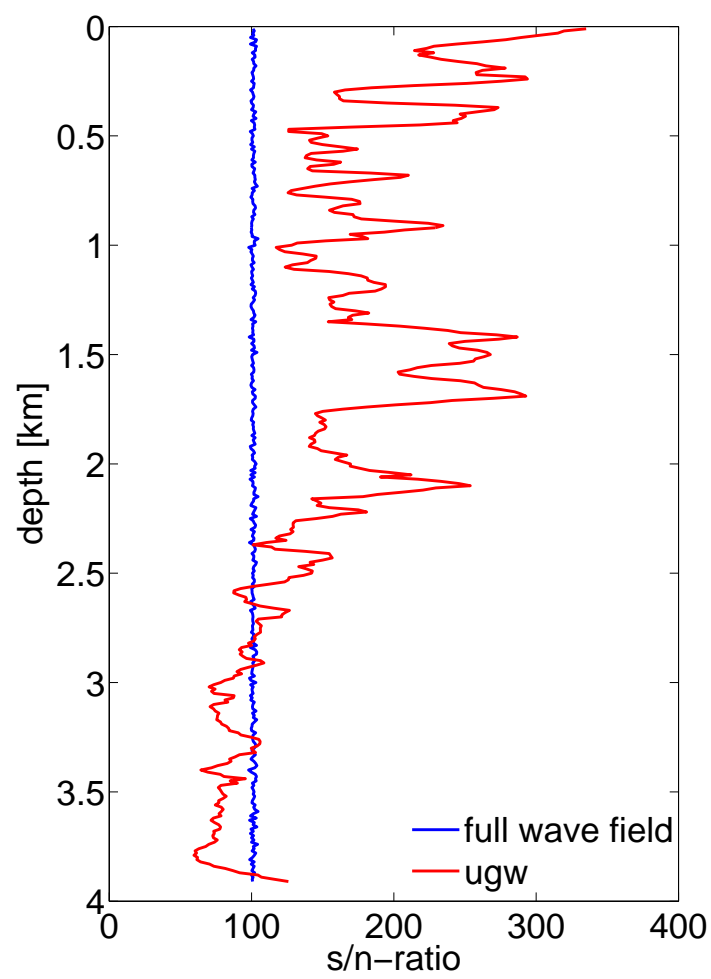

Figure 3.9: S/N-ratio of the full wavefield (blue) and after separation of up-going waves (red) of synthetic data of the Altmark model. The used f-k-filter increases the S/N-ratio for upper traces and decreases slightly for the deeper ones.

On base of the S/N-ratio, the KTB-data can be divided into 3 parts, a range of high S/N-ratios of partly over 400 in depths between $250 \mathrm{~m}$ and $900 \mathrm{~m}$, a medium part in $900 \mathrm{~m}$ to $1500 \mathrm{~m}$ with ratios around 200 and the deep part down to $3000 \mathrm{~m}$ with ratios of 20 to 40 . The overall mean ratio is 100 .

On basis of these data, noise was added to the synthetic seismograms after shifting the seismograms in time. Afterwards the wavefield separation was applied to the noisy data. Figure 3.9 shows exemplary the S/N-ratio of the full wavefield (blue) and after wavefield separation (red). It shows an increase of the S/N-ratio above about $2500 \mathrm{~m}$ while it decreases below $3000 \mathrm{~m}$. The very high values above $300 \mathrm{~m}$ are not relevant as the wavefield in this range was damped (fig. $3.7 \mathrm{c}$ ). The 
random noise has a white spectrum and so about $50 \%$ of its energy is removed during wavefield separation. This increases the $\mathrm{S} / \mathrm{N}$-ratio in the ranges above the high velocity zones, where high energetic upgoing waves (reflections, refractions) occur. Below $3000 \mathrm{~m}$, where only small impedance gradients occur and reflections are of low energy, the removing of the energy in the first quadrant (including the direct wave) decreases the overall energy of the signal stronger than the energy of the noise and the $\mathrm{S} / \mathrm{N}$-ratio decreases. The banding seen for the $\mathrm{S} / \mathrm{N}$-ratio correlates with the high energy zones in the upgoing wavefield (fig. $3.7 \mathrm{c}$ ). 


\section{Chapter 4}

\section{Amplitude changes}

The injection of $\mathrm{CO}_{2}$ induces changes in the reflection coefficient. This produces amplitude changes in the seismic wavefield. The magnitude of these amplitude changes and the depth range in which they occur are crucial information for the design of the field experiments. They depend on a number of factors, e.g.

- magnitude of changes in rock properties,

- propagation of the $\mathrm{CO}_{2}$ front and

- offset of source and receiver well.

These factors were analysed independently to understand their relativ impact. Therefore the difference between the seismograms of the initial model (without $\mathrm{CO}_{2}$ ) and the changed models (with $\mathrm{CO}_{2}$ ) was calculated and normalised to the maximum amplitude in a time window of one period of the dominant wavelength $\left(t_{w}=T_{c}\right)$ around the actual time sample. Furthermore, the impact on shifts in receiver position and on uncorrelated white noise to reflection amplitude differences was analysed.

The following figures show difference sections of $\mathrm{P}$ - and S-wavefields calculated with the FD-Program. For real data P- and S-waves can be separated by rotating the three components of the measured data (Kuzminski et al., 2009).

For this study, amplitude changes of more than $5 \%$ were expected to be measurable. This threshold is based on empirical values from marine seismic and can be applied to VSP-measurements due to similar signal-to-noise $(\mathrm{S} / \mathrm{N})$ ratios for well consolidated VSP-tools. But bad coupling, disturbing sources at the surface (especially when near to the observation well), and distinct Tube-waves can easily raise this threshold. 


\subsection{Influence of rock properties}

Changes of amplitudes in the seismic wavefield can be caused by changes in the rock properties that occur during injection, when the initial reservoir gas is replaced by $\mathrm{CO}_{2}$. Additionally pressure and temperature changes in the reservoir can influence the rock properties. The amplitude of these changes was estimated (see chapter 7) and integrated in the models.

Figure 4.1 shows amplitude changes of P- (left) and S-waves (right) for changes in rock properties of $\Delta v_{p}=-1 \%, \Delta v_{s}=-1.1 \%$ and $\Delta \rho=+1.2 \%$ (a and b), $\Delta v_{p}=-1.5 \%, \Delta v_{s}=-1.7 \%$ and $\Delta \rho=+1.8 \%$ (c and $\mathrm{d}$ ) and $\Delta v_{p}=-2 \%, \Delta v_{s}=$ $-2.25 \%$ and $\Delta \rho=+2.3 \%$ (e and f). Here $\Delta v_{p}$ are changes in P-wave velocity, $\Delta v_{s}$ are changes in $\mathrm{S}$-wave velocity and $\Delta \rho$ are changes in density. The changes were inserted in the reservoir layer of the velocity and density models shown in figure 3.3. For all three models a $\mathrm{CO}_{2}$-propagation of $500 \mathrm{~m}$ from the injection well in each direction (see fig. $4.2 \mathrm{~b}$ ) and an offset between source and observation well of $2500 m$ was chosen .

In the case of velocity changes of $-1 \%$, the amplitude changes of the direct reflected P-Wave (fig. $4.1 \mathrm{a}$ ) and in the converted PS-wave (fig. $4.1 \mathrm{~b}$ ) do not exceed the threshold of $5 \%$. And also in the later wavefield there are only marginal changes of the amplitudes. The later wavefield is of minor interest as it contains reflections at layer boundaries of greater depth and multiples. They possibly travelled through the changed region in the reservoir layer once or even several times and so they have partly larger amplitude changes than the direct reflected waves, but they can not be back-propagated to the origin of these changes with Kirchhoff migration. So in these tests we will concentrate on the direct reflected waves, which are the first onsets in the difference sections. The changes in the later wavefield will become crucial when dealing with coda-wave interferometry (chap. 6).

For $-1.5 \%$ P-wave velocity changes, amplitude changes of up to $7 \%$ occur in the direct reflected P-wave (fig. $4.1 \mathrm{c}$ ). But these changes of more than $5 \%$ can be measured only locally. The converted PS-wave (fig. $4.1 \mathrm{~d}$ ) shows amplitude changes of more than $5 \%$ in the whole depth range of $2250 \mathrm{~m}$ to $3000 \mathrm{~m}$ changes with maxima of $10 \%$. The larger effects in S-wave amplitudes may result from the larger changes in S-wave velocity.

When further increasing the velocity changes to $\Delta v_{p}=-2 \%$, amplitude changes of $7 \%$ to $8 \%$ cover a large range from $2200 \mathrm{~m}$ to $3300 \mathrm{~m}$ for the direct reflected P-wave (fig. $4.1 \mathrm{e}$ ). The converted PS-wave shows again stronger effects with amplitude changes significantly above $5 \%$ in the range between $2250 \mathrm{~m}$ and $3050 \mathrm{~m}$ with maxima of $12 \%$ (fig. $4.1 \mathrm{f}$ ). 
a)

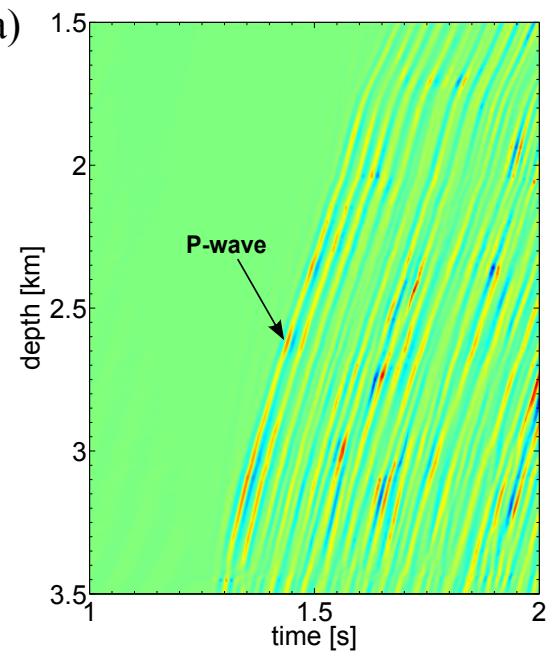

c)

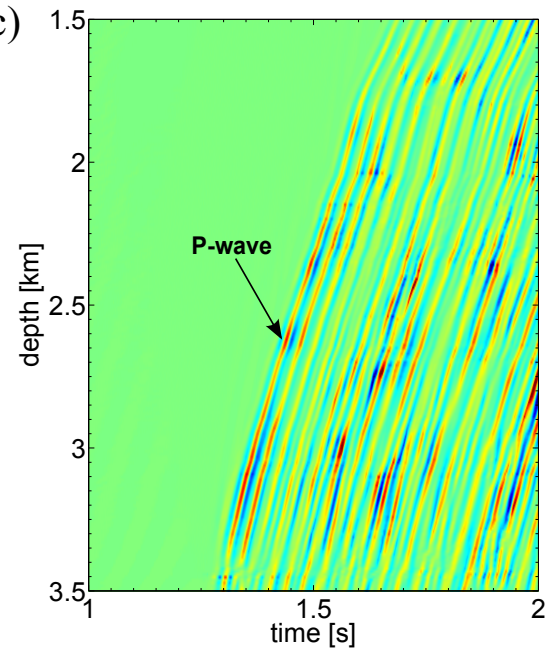

e)

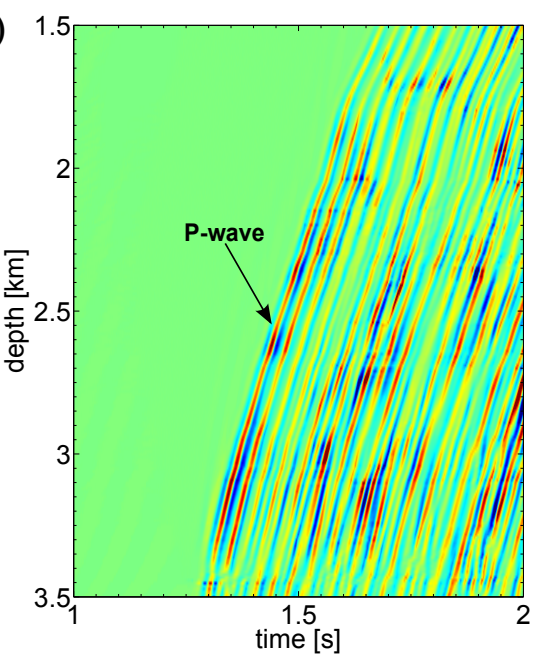

b)

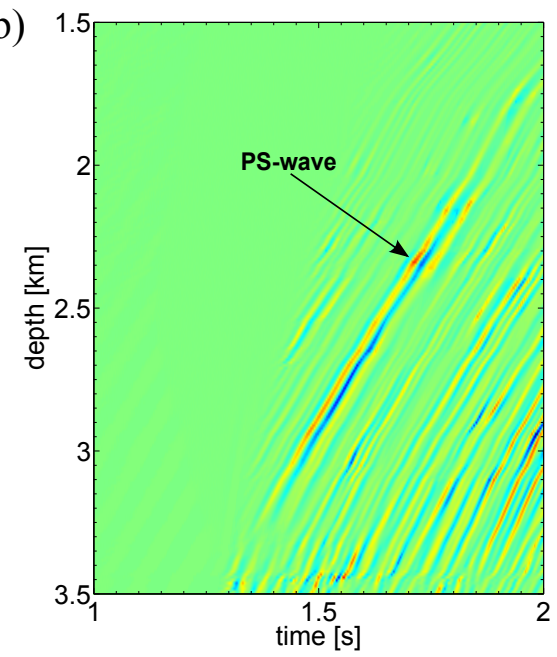

d)

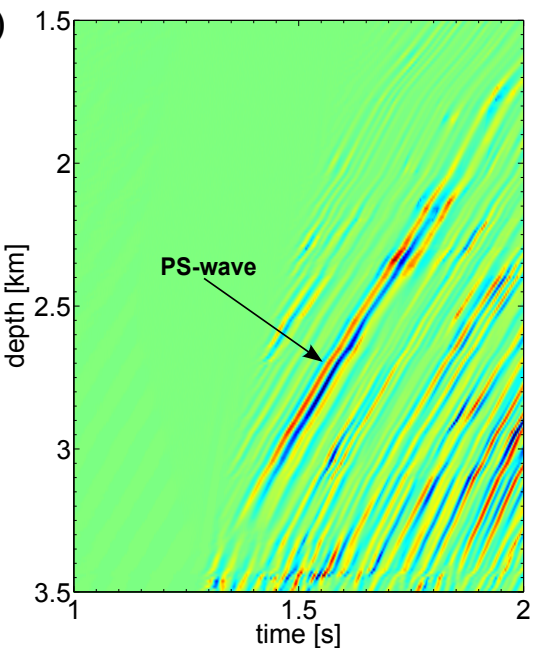

f)
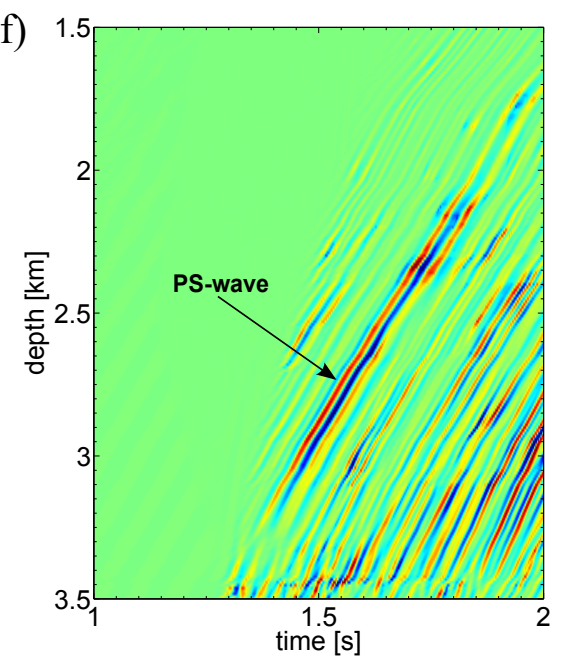

$0 \%$ $5 \%$

Figure 4.1: Amplitude changes of P-waves (left column) and S-waves (right column) for changes of rock properties of $\Delta v_{p}=-1 \%, \Delta v_{s}=-1.1 \%$ and $\Delta \rho=+1.2 \%$ ( $\mathrm{a}$ and b), $\Delta v_{p}=-1.5 \%$, $\Delta v_{s}=-1.7 \%$ and $\Delta \rho=+1.8 \%$ (c and d) and $\Delta v_{p}=-2 \%, \Delta v_{s}=-2.25 \%$ and $\Delta \rho=+2.3 \%$ (e and $\mathrm{f}$ ). 


\subsection{Propagation of the $\mathrm{CO}_{2}$-front}

Besides the strength of changes in rock properties, their lateral extent is of crucial significance. Figure 4.3 shows amplitude changes in the wavefield of $\mathrm{P}$-waves (left column) and S-waves (right column) for propagations of the $\mathrm{CO}_{2}$-front in the reservoir layer of $250 \mathrm{~m}$ ( $\mathrm{a}$ and b), $500 \mathrm{~m}$ (c and d) and $1000 \mathrm{~m}$ (e and f). All three models are calculated for a source-receiver offset of $2500 \mathrm{~m}$ and changes of rock properties of $\Delta v_{p}=-2 \%, \Delta v_{s}=-2.25 \%$ and $\Delta \rho=+2.3 \%$. The extension of the $C_{2}$ in the reservoir for the three models is shown in figure 4.2 .
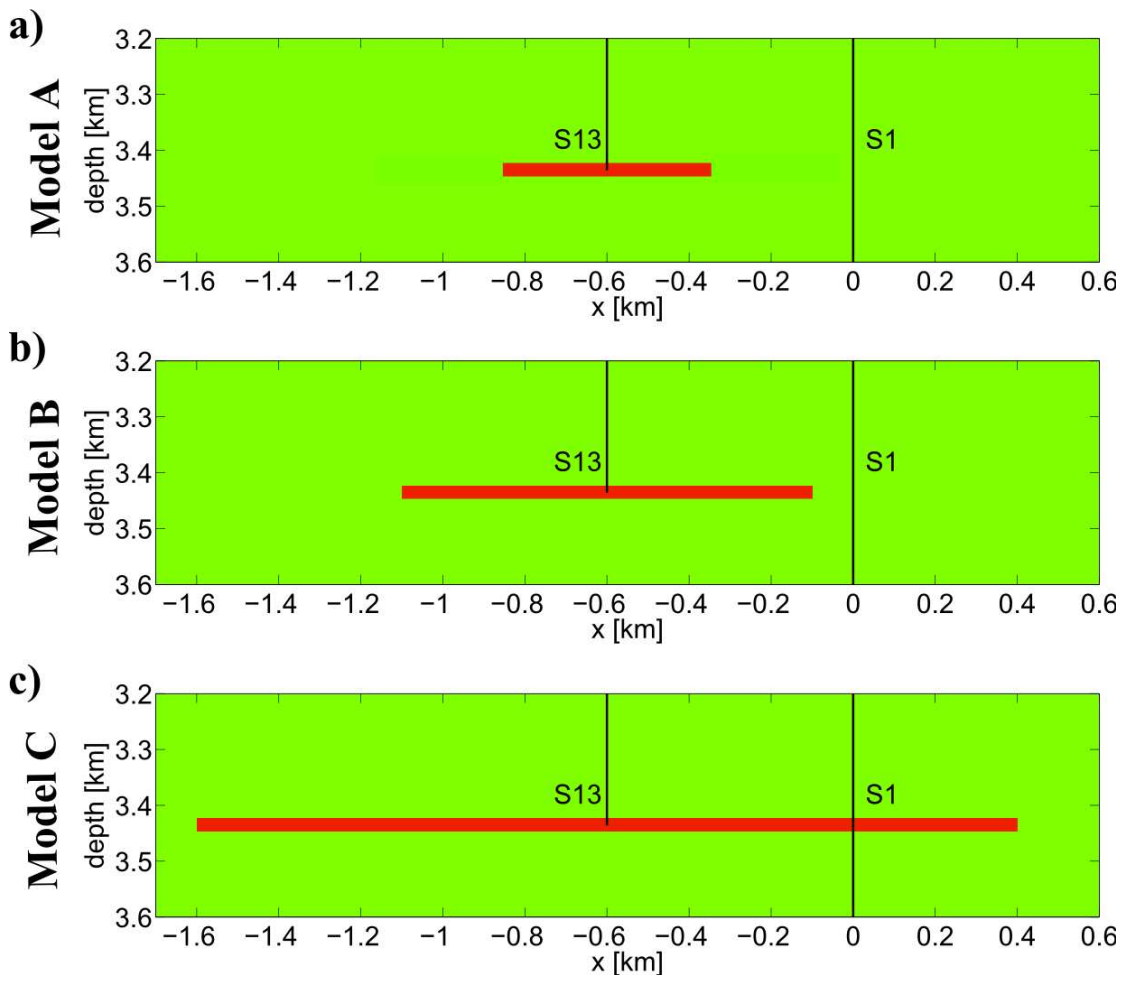

Figure 4.2: Difference in velocity models indicating the velocity changed zones. Model A shows a $\mathrm{CO}_{2}$-propagation of $250 \mathrm{~m}$, Model $\mathrm{B}$ of $500 \mathrm{~m}$ and Model $\mathrm{C}$ of $1000 \mathrm{~m}$.

The effects of an extension of the $\mathrm{CO}_{2}$ cloud of $250 \mathrm{~m}$ around the injection well (Model A in fig. 4.2) amplitude changes of the direct reflected $\mathrm{P}$-wave are larger than $5 \%$, with a maximum of about $10 \%$ (fig. 4.3 a) for the major part of the range from $2450 \mathrm{~m}$ to $2950 \mathrm{~m}$. The S-wave shows less clear effects with only very local amplitude changes of more than $5 \%$ (fig. $4.3 \mathrm{~b}$ ).

Figures $4.3 \mathrm{c}$ ) and d) show amplitude changes for a propagation radius of $500 \mathrm{~m}$ (Model B in fig. 4.2). These figures are equivalent to figures $4.1 \mathrm{e}$ ) and f). Compared to the $250 \mathrm{~m}$ offset, for P-waves mainly the depth range, where significant amplitude changes occur, changes, while the maximum amplitude changes are again at about 
a)

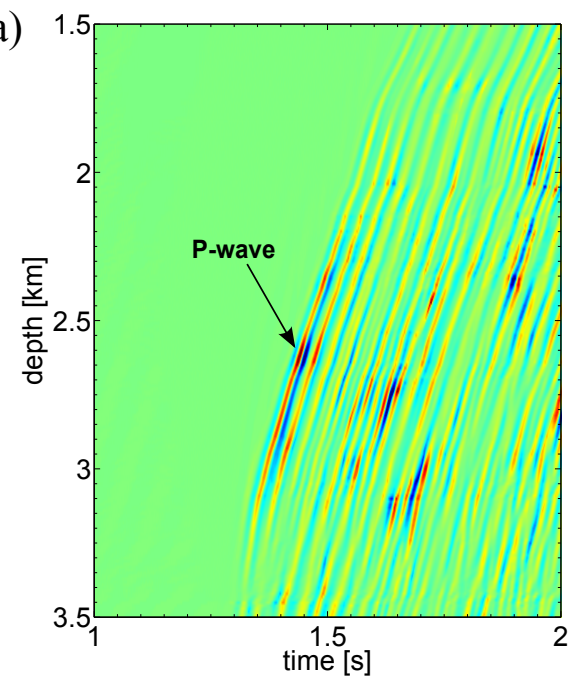

c)

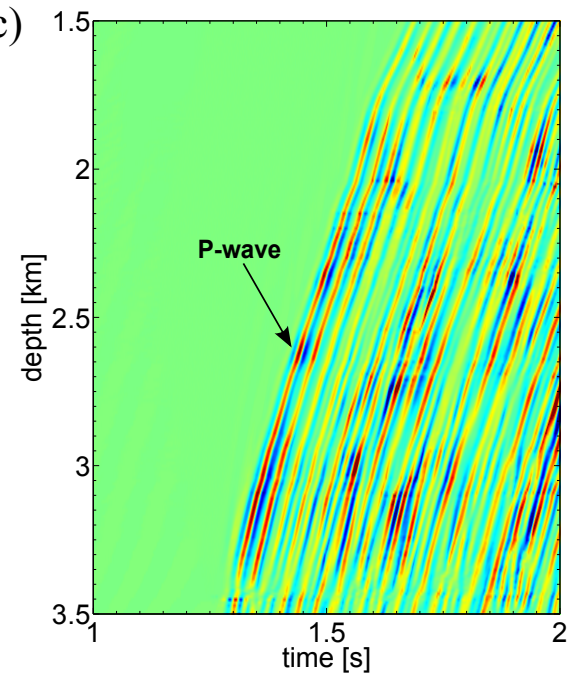

e)

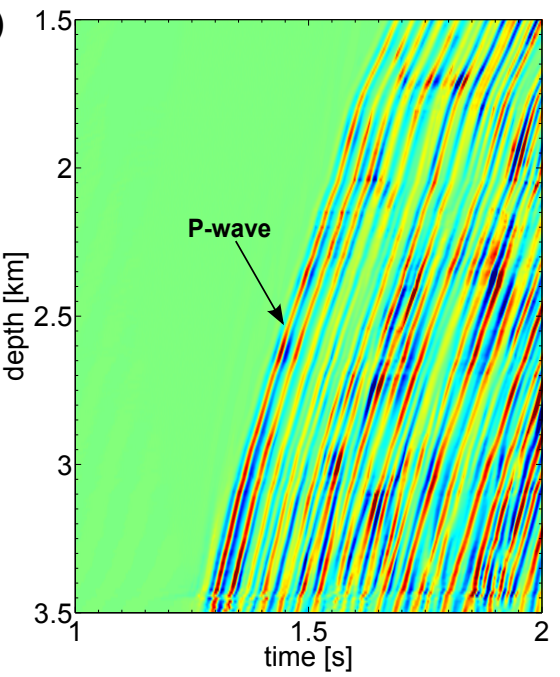

b)

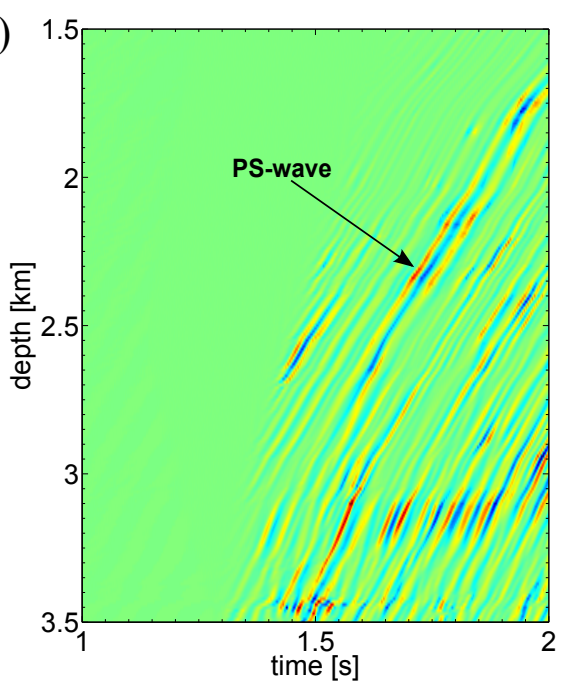

d)

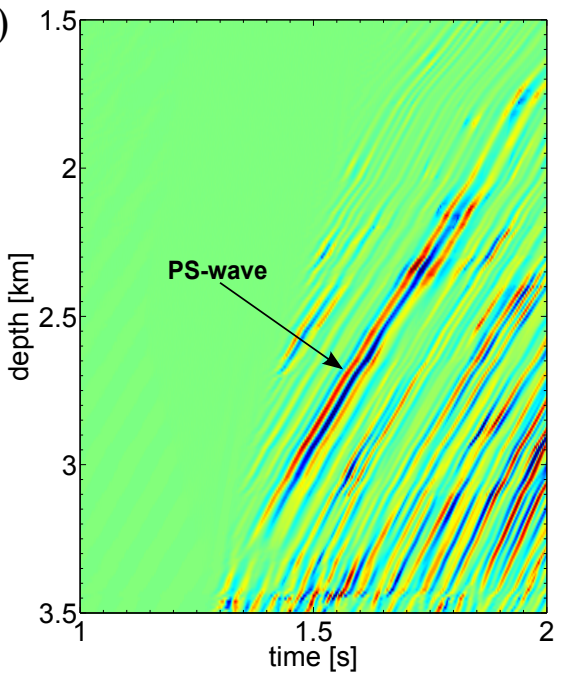

f)

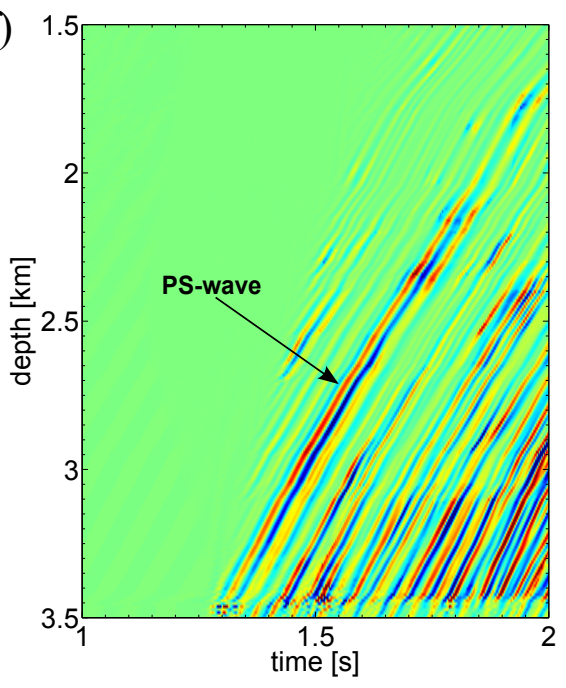

$\begin{array}{lllll}-10 \% & -5 \% & 0 \% & 5 \% & 10 \%\end{array}$

Figure 4.3: Amplitude changes of P-waves (left column) and S-waves (right column) for propagation of the $\mathrm{CO}_{2}$-front of $250 \mathrm{~m}$ (a and b), $500 \mathrm{~m}$ (c and d) and $1000 \mathrm{~m}$ (e and f). 
$10 \%$ (fig. $4.3 \mathrm{c}$ ). However, the doubling of the propagation radius has a conspicuous effect to S-waves. They show now significant amplitude changes of up to $12 \%$ in an extended depth range (fig. $4.3 \mathrm{~d}$ ).

In the case that the radius is of $1000 \mathrm{~m}$, a breakthrough at the observation well occurred which has an offset of $600 \mathrm{~m}$ to the injection well (fig. $4.2 \mathrm{c}$ ). Now, for Pas well as for S-waves, the lower boundary of the depth range, in which significant amplitude changes can be observed, is shifted down to the reservoir depth (ca. $3425 \mathrm{~m}$ ). The upper boundary as well as the maximum changes are nearly unaffected and remain the same as for $500 \mathrm{~m}$ (fig. $4.3 \mathrm{e}$ und f).

\section{$4.3 \quad$ Source-receiver offset}

The influence of the source-receiver offset on the strength and distribution of the amplitude changes was tested. Figure 4.4 shows the difference sections of P-and S-waves for offsets of $1000 \mathrm{~m}$ ( $\mathrm{a}$ and b), $2500 \mathrm{~m}$ (c and d) and $4000 \mathrm{~m}$ (e and $\mathrm{f}$ ). In all of these models the radius of the $\mathrm{CO}_{2}$-cloud is $500 \mathrm{~m}$ and the changes of rock properties are $\Delta v_{p}=-2 \%, \Delta v_{s}=-2.25 \%$ and $\Delta \rho=+2.3 \%$.

For small offsets the largest amplitude changes in the direct reflected $\mathrm{P}$-wave occur in shallow depth between $1200 \mathrm{~m}$ and $2300 \mathrm{~m}$ (fig. $4.4 \mathrm{a}$ ). The changes are small at approximately 5\%. And also the amplitudes of the converted PS-wave change only above $2300 \mathrm{~m}$ with only small maxima.

The difference sections for an offset of $2500 \mathrm{~m}$ were already discussed in the previous tests and show changes of $7 \%$ to $8 \%$ between $2200 \mathrm{~m}$ and $3300 \mathrm{~m}$ for the P-wave (fig. $4.4 \mathrm{c}$ ) and changes of $12 \%$ in maximum between $2250 \mathrm{~m}$ and $3050 \mathrm{~m}$ for the converted PS-wave (fig. $4.4 \mathrm{~d}$ ).

For very large source-receiver offsets of $4000 \mathrm{~m}$ the depth range, in which amplitude changes occur, becomes much smaller. In the case of the direct reflected P-wave it ranges from $2700 \mathrm{~m}$ to $3350 \mathrm{~m}$. The maximum changes are up to $11 \%$. The changes in the difference sections of the shear waves extend from $2500 \mathrm{~m}$ down to the reservoir in $3425 \mathrm{~m}$. They show strong amplitude changes of up to $10 \%$.

\subsection{Noise}

In field experiments one never has optimum conditions and real data will always contain a certain amount of noise. In this chapter the effect of noise on the interpretation of amplitude changes is analyzed answering the question under which conditions the amplitude changes, resulting from impedance changes in the reservoir, still can be observed. Therefore the simulated data are disturbed with white 
a)

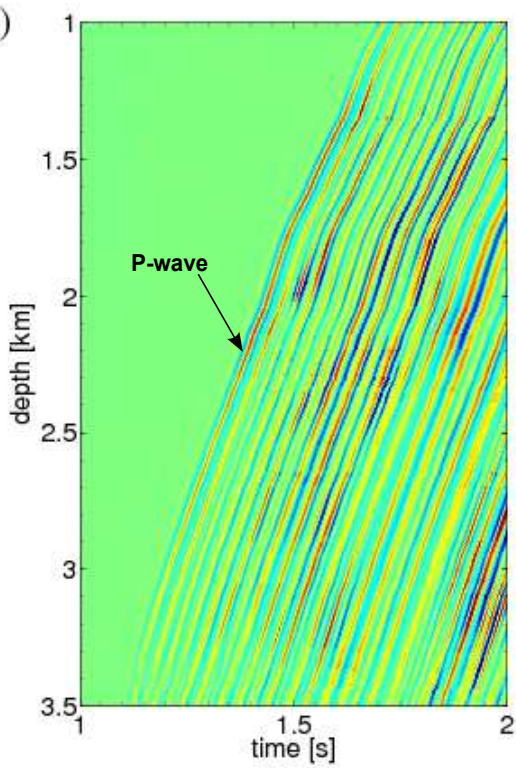

c)

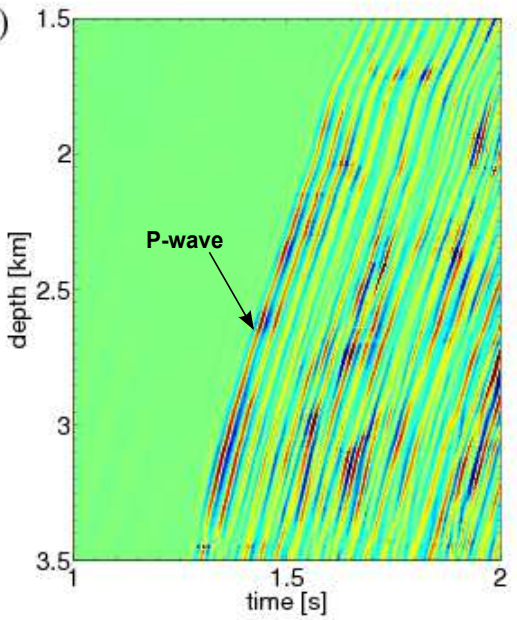

e)

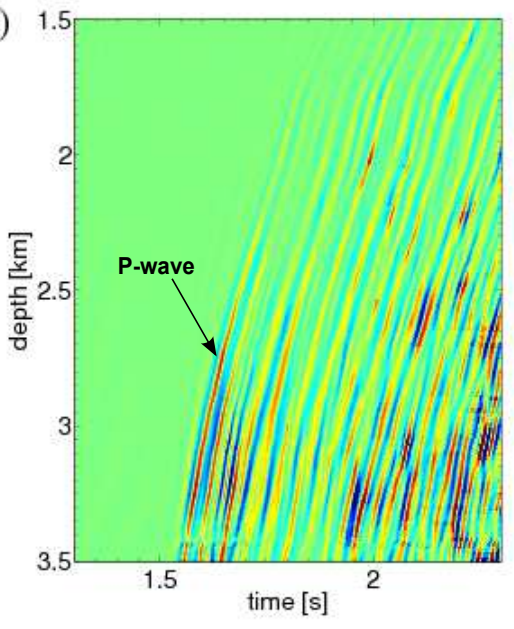

$-10 \% \quad-5 \%$ b)

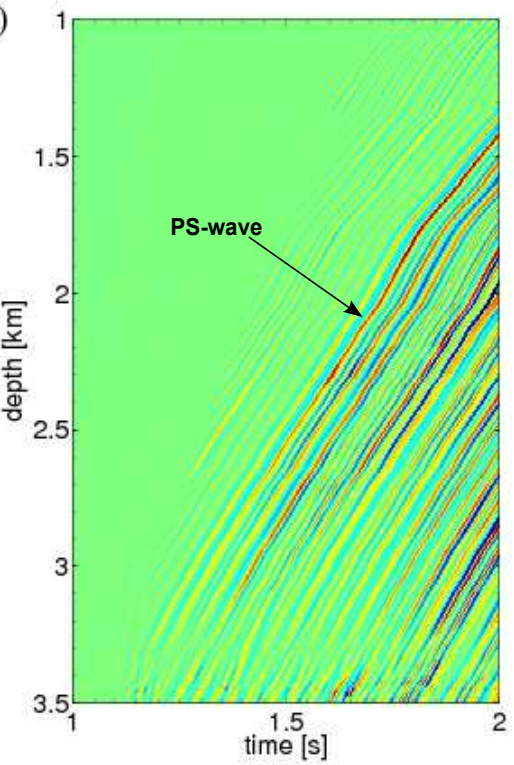

d)

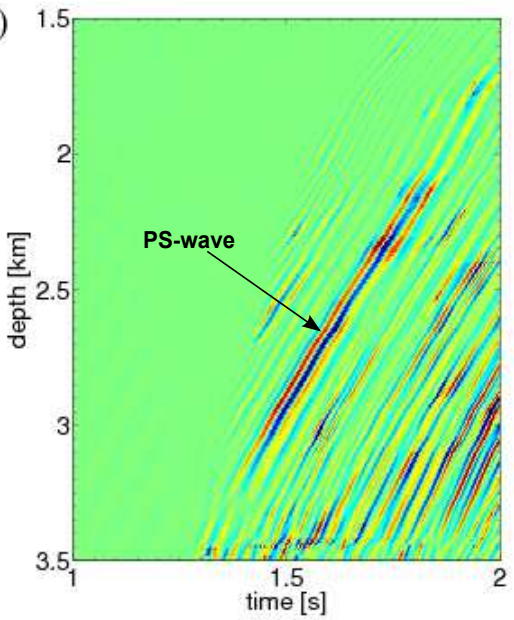

f)

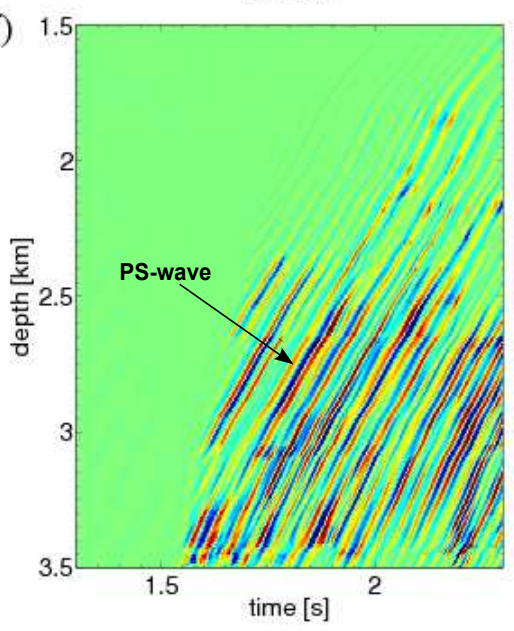

$0 \% \quad 5 \% \quad 10 \%$

Figure 4.4: Amplitude changes of P-waves (left column) and S-waves (right column) for offsets between source and geophone chain of $1000 \mathrm{~m}$ ( $\mathrm{a}$ and b), $2500 \mathrm{~m}$ (c and d) and $4000 \mathrm{~m}$ (e and f). 
noise (random signal) of different Signal-to-Noise (S/N) ratios as discribed in chapter 3.3.3. Figure 4.5 shows the difference sections with the same conditions as for figure $4.1 \mathrm{e}$ ) and f) but with S/N-ratios of 100 (fig. $4.5 \mathrm{a}$ and b), 40 (fig. $4.5 \mathrm{c}$ and d) and 20 (fig. 4.5 e and f).

Strong changes occur in the region before the first break, where zeros appeared in the noise-free data. After this the differences in the direct reflections from the reservoir depth are still good to identify for P-waves as well as for S-waves at a high $\mathrm{S} / \mathrm{N}$-ratio of 100 (fig. $4.5 \mathrm{a}$ and b). But parts of the coda are already disguised by noise.

For a $\mathrm{S} / \mathrm{N}$-ratio of 40 , multiples are completely overlaid by differences resulting from noise. Changes in the direct reflection from reservoir depth is hard to obtain in $\mathrm{P}$-waves but not overlaid over the complete depth range (fig. $4.5 \mathrm{c}$ ). The $\mathrm{S}$ wave is not affected that strongly (fig. $4.5 \mathrm{c}$ ). Here the changes in the converted PS-reflection still have to be identified.

When further decreasing the $\mathrm{S} / \mathrm{N}$-ratio to 20 it becomes impossible to identify the injection induced amplitude changes in up-going P- (fig. 4.5 e) and PS-waves (fig. $4.5 \mathrm{f}$ ). They are much smaller than the overlaid noise fluctuations.

\subsection{Shift}

Beside noise, a typical problem in time-lapse VSP-experiments occur from an imperfect reinstallation of the VSP-tools.

The impact of a shift in the position of the receivers during the repeat measurement relative to the baseline was analysed. Therefore the position of all receivers was shifted by $1 m$ (1 grid-point) in the model with $\Delta v_{p}=-2 \%, \Delta v_{s}=-2.25 \%$ and $\Delta \rho=+2.3 \%$, a $500 \mathrm{~m}$ propagation radius and a source offset of $2500 \mathrm{~m}$. The result is shown in figure 4.6 for P-waves (a) and S-waves (b).

It shows that shifts have much greater effects on S-waves, which are mainly contained in the horizontal component in this configuration. Here the changes resulting from the imperfect repeat of the experiment configuration are in the same dimension or larger than the injection induced changes. Under these conditions an interpretation of $\mathrm{S}$-waves is not possible.

P-waves are less affected. Here the injection induced changes are still dominant. The strongest amplitude changes occur at the same depth range and time as in the "perfect repeat experiment" in figure $4.1 \mathrm{e}$ ). But the dimension of the changes decreased in the direct reflected P-wave and are now mainly between 5\% and 6\% with a maximum of $9 \%$. Only slightly minor changes occur in the whole up-going wavefield, also before the direct reflection from reservoir depth. This makes a clear 
a)

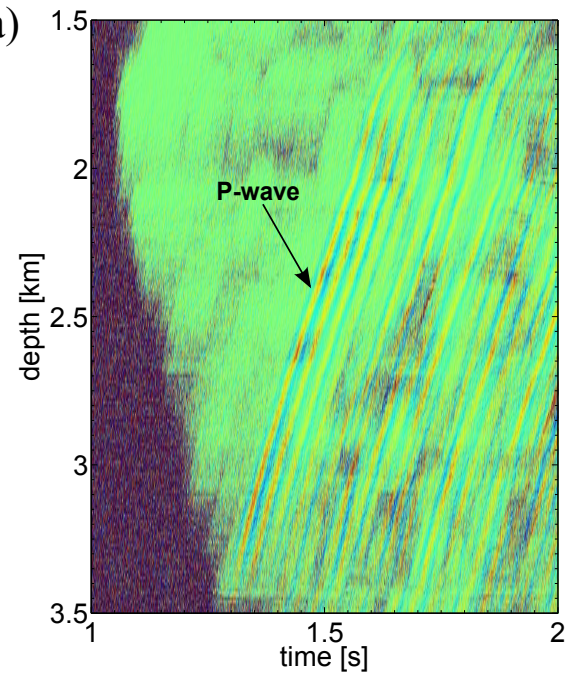

c)

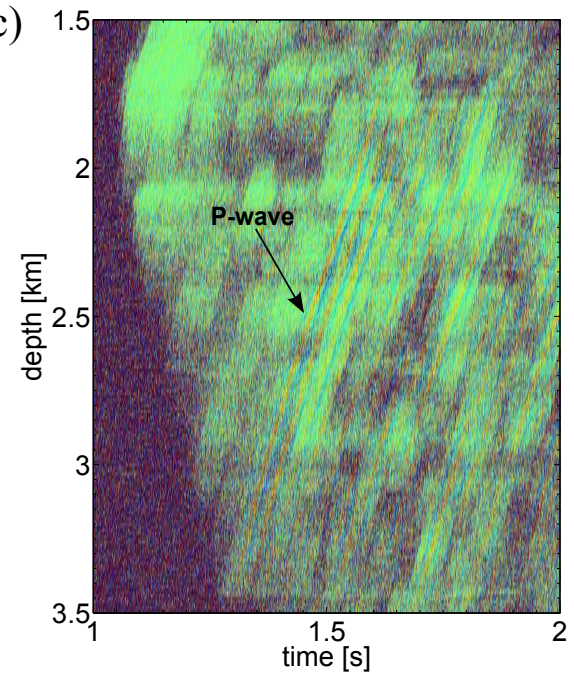

e)

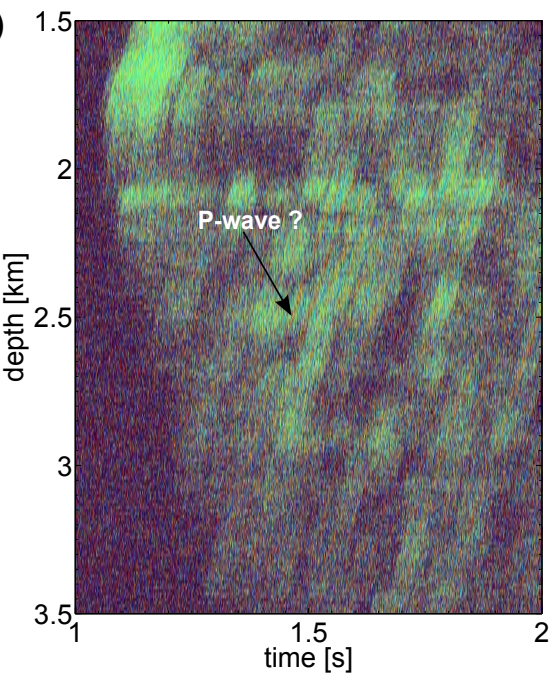

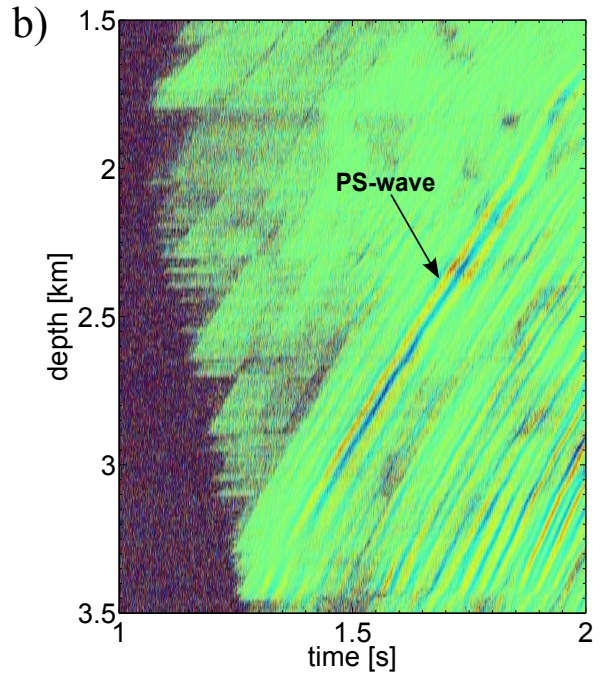

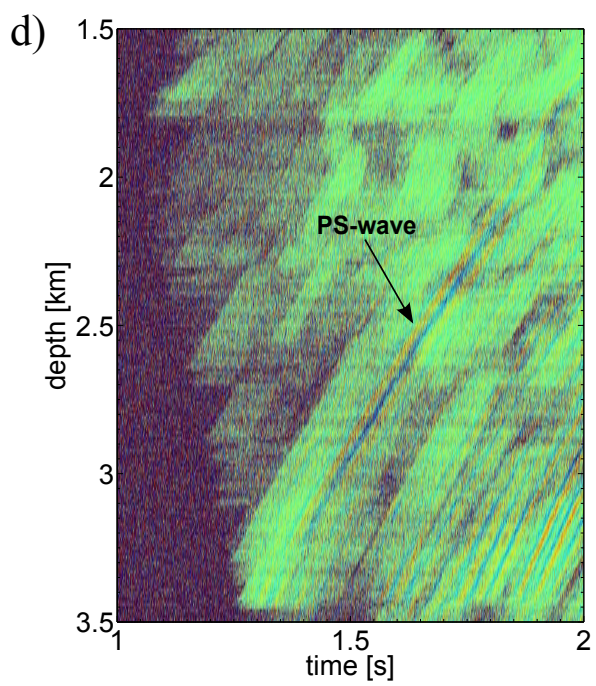

f)

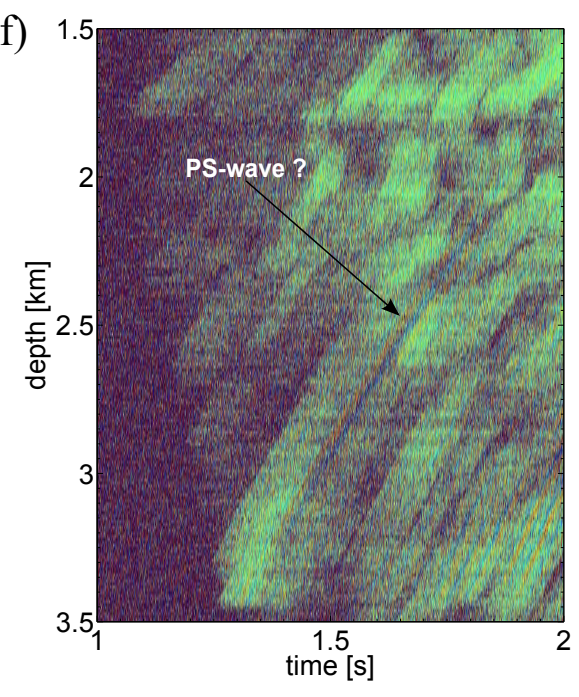

Figure 4.5: Amplitude changes of P-waves (left column) and S-waves (right column) polluted with noise of S/N-ratios of 100 ( $a$ and b), 40 (c and d) and 20 (e and f). 
a)

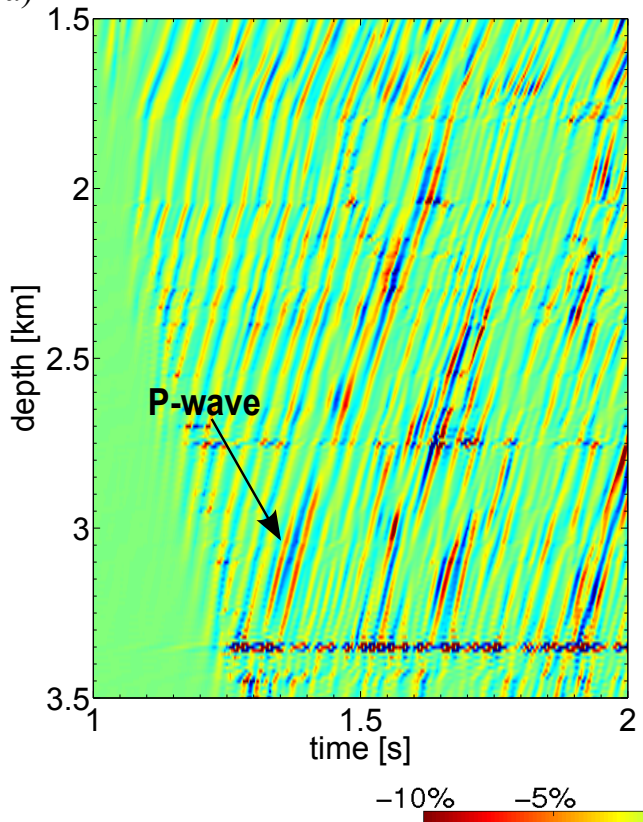

b)

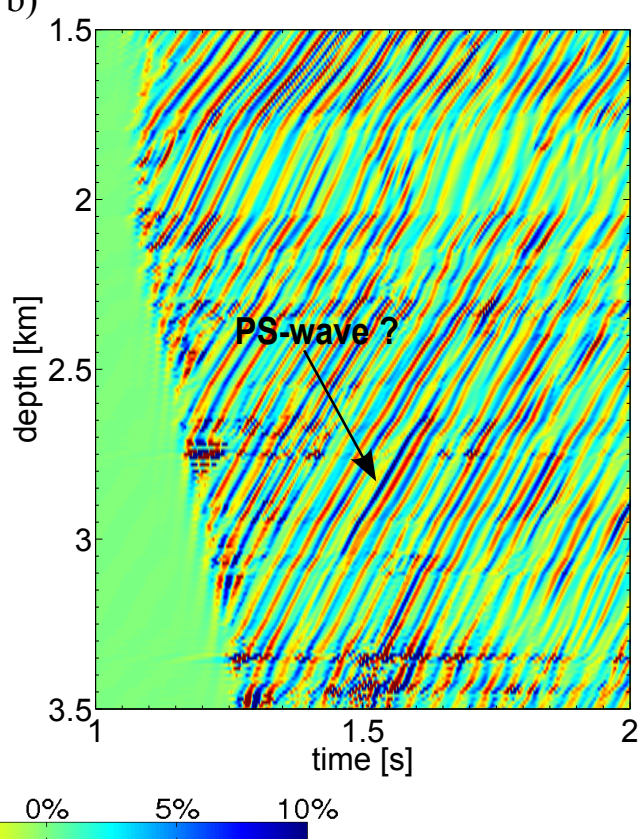

Figure 4.6: Amplitude changes of P-waves (a) and S-waves (b) for a shift of the receiver of $1 \mathrm{~m}$.

identification and an exclusion of $\mathrm{CO}_{2}$ accumulation in shallower depth (leakage) very difficult.

\subsection{Conclusion}

The evaluation of amplitude changes in the seismic wavefield results in a minimum of $-1.5 \% \mathrm{P}$-wave velocity changes and corresponding density and S-wave velocity changes are necessary to produce amplitude changes of more than $5 \%$ in $\mathrm{P}$ - as well as in converted PS-waves. Amplitude changes of 5\% were set as a threshold for VSP-experiments. Noise tests showed that in data of S/N-ratios of about 40 or higher an identification of amplitude changes of about $5 \%$ would be possible, while $\mathrm{S} / \mathrm{N}$-ratios below 40 would make an identification difficult. The field tests at the KTB showed that 40 is a low but realistic S/N-ratio for deep VSP-surveying (fig. 3.8). So the noise tests suggest $5 \%$ to be a realistic threshold.

Shifts in receiver position of $1 \mathrm{~m}$ produce artefacts in the whole wavefield. They widely do not exceed about half of the injection induced amplitude changes for Pwaves. But in S-wavefields they do produce larger effects. Here injection induced amplitude changes in the converted PS-wave can not to be identified any more.

The propagation of the $\mathrm{CO}_{2}$-front is displayed in the difference section by the range of maximum amplitude changes. The well range available for the field ex- 
periments $(2200 m-3300 m)$ is sufficient to monitor most of the large amplitude changes for propagations up to $1000 \mathrm{~m}$ with medium source-receiver offsets. To cover the very near part of the reservoir small source offsets have to be chosen.

The tests further showed that large offsets $(4000 \mathrm{~m})$ slightly increase the amplitude changes by focusing them in the region above the reservoir. Small offsets lead to the opposite effect. They shift amplitude changes to shallower depth and smear them over a larger range. This will become useful, when the $\mathrm{CO}_{2}$-cloud comes near to the observation well and the lower depth limit of measurable amplitude changes approaches the maximum receiver depth at $3300 \mathrm{~m}$.

But it has to be kept in mind, that these test were calculated without attenuation and damping can decrease the data quality (S/N-ration, frequency content, etc.) significantly, especially for far offsets.

However, the survey design for the field experiment was planned based on these information. They are also basis of the migration tests in the next chapter. 



\section{Chapter 5}

\section{Migration}

It is of great interest, to use the amplitude changes calculated in the previous chapter to image the corresponding changes in rock properties in the depth section. To test whether this is possible or not, standard Kirchhoff pre-stack depth migration and Fresnel-Volume migration were used. In the first part the theory of Fresnel-Volume migration is explained and then the migration results are shown and analysed.

\subsection{Theory}

Kirchhoff pre-stack depth migration (Kirchhoff PSDM) is a weighted diffraction stack. The migrated image $V(\vec{r})$ at a point $\vec{r}$ in the subsurface is given by weighted summation along diffraction surfaces

$$
V(\vec{r})=\iint_{A} W\left(\vec{r}, \vec{r}^{\prime}\right) \dot{u}\left(\vec{r}^{\prime}, t_{S}+t_{R}\right) d \vec{r}^{\prime},
$$

where $t_{S}$ and $t_{R}$ are travel times from the source $(\mathrm{S})$ and the receiver $(\mathrm{R})$ to the image point $\mathrm{M}$. W $\left(\vec{r}, \vec{r}^{\prime}\right)$ is a weighting function and accounts for the correct treatment of amplitudes during back propagation. $\vec{r}^{\prime}$ are locations of sources and receivers at the surface. They constitute the acquisition surface A which is a vertical line in the case of VSP-experiments, but can also be an arbitrary shaped surface in the case of 3D surface seismics. $\dot{u}$ describes the time derivative of the seismic wavefield recorded at the geophones. The derivative is needed to recover the source pulse correctly (Newman, 1975). For data recorded with two or three components, the wavefield has to be projected to the direction of its polarization which is given by a scalar product $\left(\dot{\vec{u}}\left(\vec{r}^{\prime}, t_{S}+t_{R}\right) \cdot \vec{e}_{r}\right)$. This is of course only valid for the special treatment of P-to-P-reflections. The principles of standard Kirchhoff pre-stack depth migration are described e.g. in Schleicher et al. (1993).

Fresnel-Volume Migration is an extension of the established Kirchhoff depth migration. It restricts the recorded reflection to that point of the migrated image 
that contributes to the signal. This can be achieved by adding a second weight function $W_{F}$ to equation 5.1 giving

$$
V(\vec{r})=\iint_{A} W_{F}\left(\vec{r}, \vec{r}^{\prime}, t_{S}+t_{R}\right) W\left(\vec{r}, \vec{r}^{\prime}\right) \dot{\vec{u}}\left(\vec{r}^{\prime}, t_{S}+t_{R}\right) \cdot \vec{e}_{r} d \vec{r}^{\prime} .
$$

The weight function restricts the smearing of the wavefield to the Fresnel volume at the actual reflection point instead of smearing it along the complete TWT isochrone, as it is done in standard Kirchhoff depth migration (fig. 3 in Lüth et al.,2005). It is defined by

$$
W_{F}\left(\vec{r}, \vec{r}^{\prime}, t_{S}+t_{R}\right)\left\{\begin{array}{rr}
1: & r_{I} \leq r_{Q}, \\
1-\frac{r_{I}-r_{Q}}{r_{Q}}: & r_{Q}<r_{I} \leq 2 r_{Q}, \\
0: & r_{I} \geq 2 r_{Q},
\end{array}\right.
$$

where $r_{Q}$ is the radius of the first Fresnel zone at ray point $Q$ and $r_{I}$ is the nearest distance from the ray to the image point under consideration (Sick, 2005).

The Fresnel volume in a homogeneous velocity model can be calculated by use of geometric considerations. A reflection point D lies within the Fresnel zone of the reflection point $\mathrm{M}$, if its travel time $t(S, D)+t(D, R)$

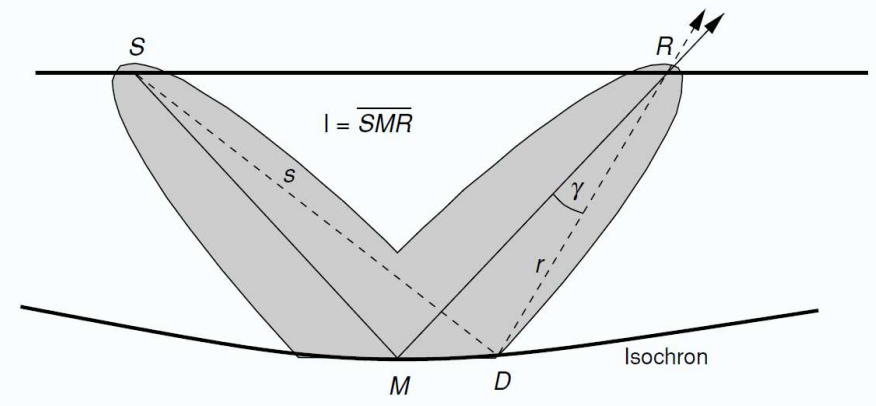

Figure 5.1: Construction of the Fresnel volume in a homogeneous medium. The black line denotes the surface of constant TWT traveltime for reflection point $\mathrm{M}$ and the grey area shows the Fresnel zone (Lüth et al., 2005). differs not more than half a period from the travel time through the reflection point under consideration $t(S, M)+$ $t(M, R)$

$$
|t(S, D)+t(D, R)-t(S, M)-t(M, R)| \leq n \frac{T}{2}[n=1,2, \ldots],
$$

where $T$ is the dominant period and $n$ is the order of the Fresnel volume (Sick, 2005). This can be expressed in the case of a homogeneous model and a monofrequent source signal by

$$
|s+r-l| \leq n \frac{\lambda}{2}[n=1,2, \ldots]
$$

where $l$ is the length of the raypath $\overline{S M R}$ and $s$ and $r$ are the length of the raypaths from the scatter point $\mathrm{D}$ to the source $(\overline{S D})$ and receiver $(\overline{D R})$, respectively (fig. $5.1)$. 


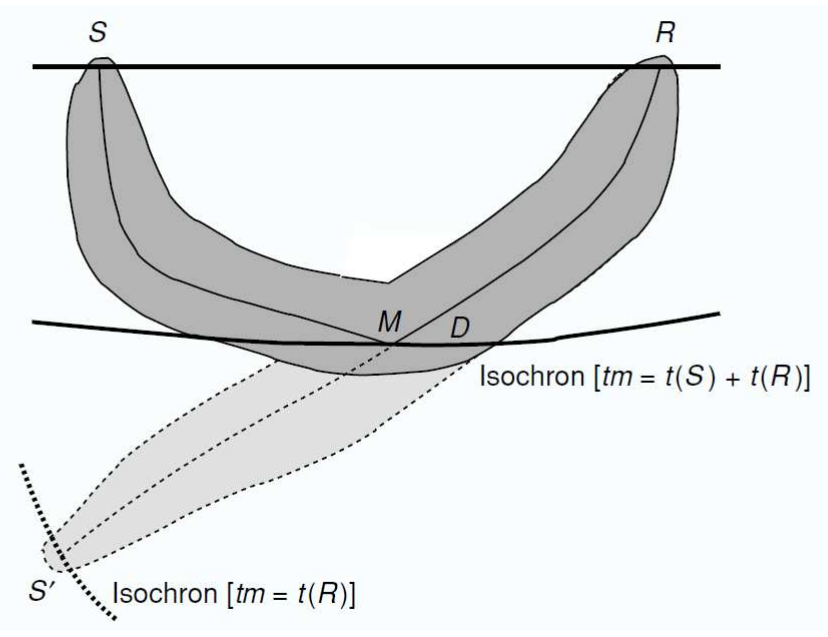

Figure 5.2: Calculation of the Fresnel volume in a heterogeneous medium by direct ray path construction from the receiver to the virtual mirror point $S^{\prime}$. The Fresnel volumes are identical at the isochrone for smooth velocity models (Lüth et al., 2005).

structed by a computational costly two-point ray tracing. Therefore the used migration program approximates the Fresnel volume of the raypath $\overline{S M R}$ with the direct ray from the receiver to the virtual mirror source $S^{\prime}$ (fig. 5.2). For the part from the reflection point to the receiver it is identical for both rays. For smooth velocity models the Fresnel volume is given by (Lüth et al., 2005)

$$
r_{Q} \approx \sqrt{\frac{T}{\frac{1}{\Pi_{13}(Q)}-\frac{1}{\Pi_{13}(Q) \Pi_{13}\left(S^{\prime}\right)}}}
$$

and has a circular shape. $\Pi_{13}$ are elements of the ray propagator matrix $\Pi$ at the point under consideration on the ray $Q$ and at the end of the ray $S^{\prime}$, respectively. $\Pi_{13}$ is given by (Lüth et al., 2005)

$$
\frac{d \Pi_{13}}{d t}=v^{2} .
$$

Strictly speaking, this description of the Fresnel zone is restricted to sufficiently smooth velocity models. But also nonvanishing velocity contrasts only result in slightly wrong estimations of the size of the Fresnel volume, while its position is not affected (Buske et al., 2009; Lüth et al., 2005). And as migration velocity models are generally smooth and the subpression of migration artifacts is preceeding, small inaccuracies in the size of the Fresnel zone can be tolerated.

Further the computation of the correct polarization of the wavefield at the receiver is very important as it defines the center of the Fresnel zone. For the shown 
models a slant stack method was used to calculated the correct slowness at the receivers.

\subsection{Migration}

By means of migration methods it was tested if and under which conditions amplitude changes in the seismic sections can be used to image the corresponding changes in rock properties in the depth section. Therefore the difference sections for 11 different source positions with offsets between $500 \mathrm{~m}$ and $5500 \mathrm{~m}$ were calculated (fig. 3.3). The difference sections of receivers between $2200 \mathrm{~m}$ and $3300 \mathrm{~m}$ were used. This is the depth range that is available in the observation well. This limitation is on the one hand given by the plug, that has to be set in the bottom (perforated) part of the well, and on the other hand by an uncemented tubing in the upper part of the observation well S1 where the recording of seis-

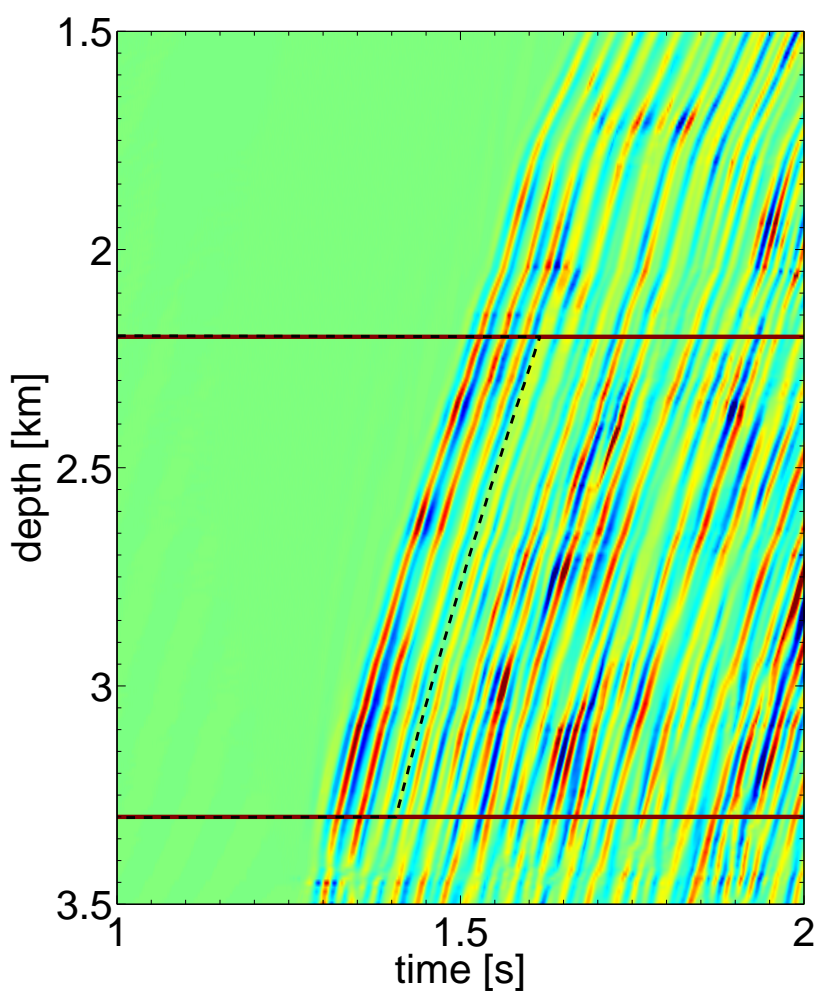

Figure 5.3: Difference section of synthetic seismic data. The red lines display the limits that can be used for VSPseismic in the observation well in the Altmark. The data were cut along the dashed line in order to separate the primary data from deeper reflections and multiples. mic signals will be impossible.

Figure 5.3 shows the migrated range of the difference section for the model with $\Delta v_{p}=-2 \%, \Delta v_{s}=-2.25 \%$ and $\Delta \rho=+2.3 \%$, a $500 \mathrm{~m}$ propagation radius of the $\mathrm{CO}_{2}$-cloud and a source offset of $2500 \mathrm{~m}$.

The ray tracing in Fresnel-Volume migration requires a sufficiently smooth velocity model. The original model from figure 3.3 has too strong gradients, so that it was smoothed with a $100 \mathrm{~m}$ broad triangle function (fig. 5.4). This still caused problems with receivers above $2000 \mathrm{~m}$ due to guided waves between the high velocity zones in $1400 \mathrm{~m}$ and $1900 \mathrm{~m}$, but worked well within the used receiver range. Even though 
damping of the velocity model is not necessary in Kirchhoff migration this smoothed model was also used for KPDM, in order to simulate a not perfectly known velocity model. As input for the migration only the difference sections (amplitude changes) of P-waves were used.

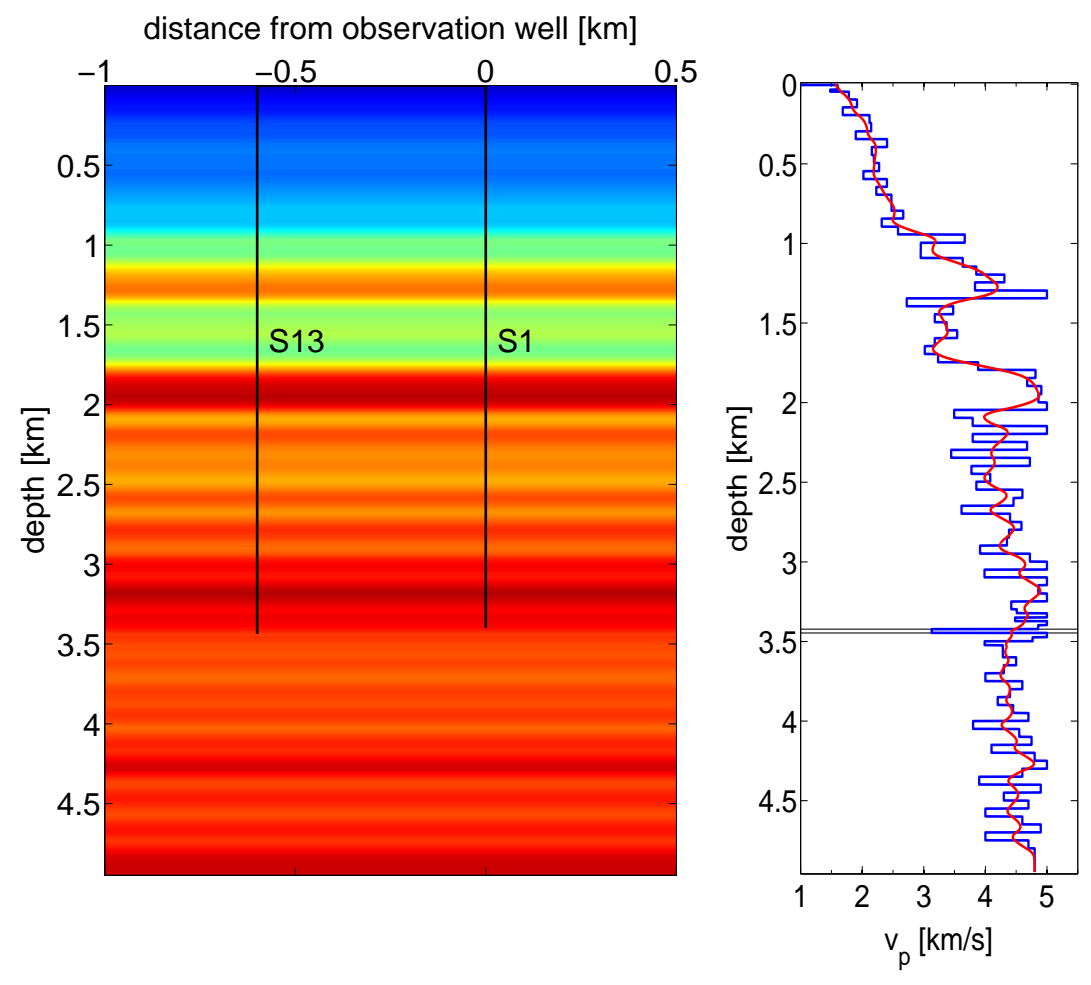

Figure 5.4: Smoothed velocity model used for miration.

\subsubsection{Kirchhoff migration}

In a first step these data were imaged to the depth section with standard Kirchhoff migration for the difference sections from all 11 source positions. The hole $4 \mathrm{~s}$ traces (region between red lines in figure 5.3) were used for the migration. The migration result is shown in figure 5.5. S1 is the observation well, S13 the injection well and the dashed lines indicate the region, where the changes were implemented to the velocity model.

The changes in the migrated section can reproduce the dimension of the $\mathrm{CO}_{2^{-}}$ cloud very well and are only slightly shifted to greater depth. This can be explained with the incorrect, because smoothed, velocity model. Weaker reflections appear below the first strong reflector. They suggest that not exclusively direct reflections from the top reservoir, but also multiples and reflectins from layer boundaries below, which passed through the region with changed rock properties at least once, were 
migrated. This can lead to the wrong conclusion of $\mathrm{CO}_{2}$ accumulations in greater depth as it was also shown by Lumley (2010).

However, all reflections occur below the top reservoir and $\mathrm{CO}_{2}$-leakages can be excluded.

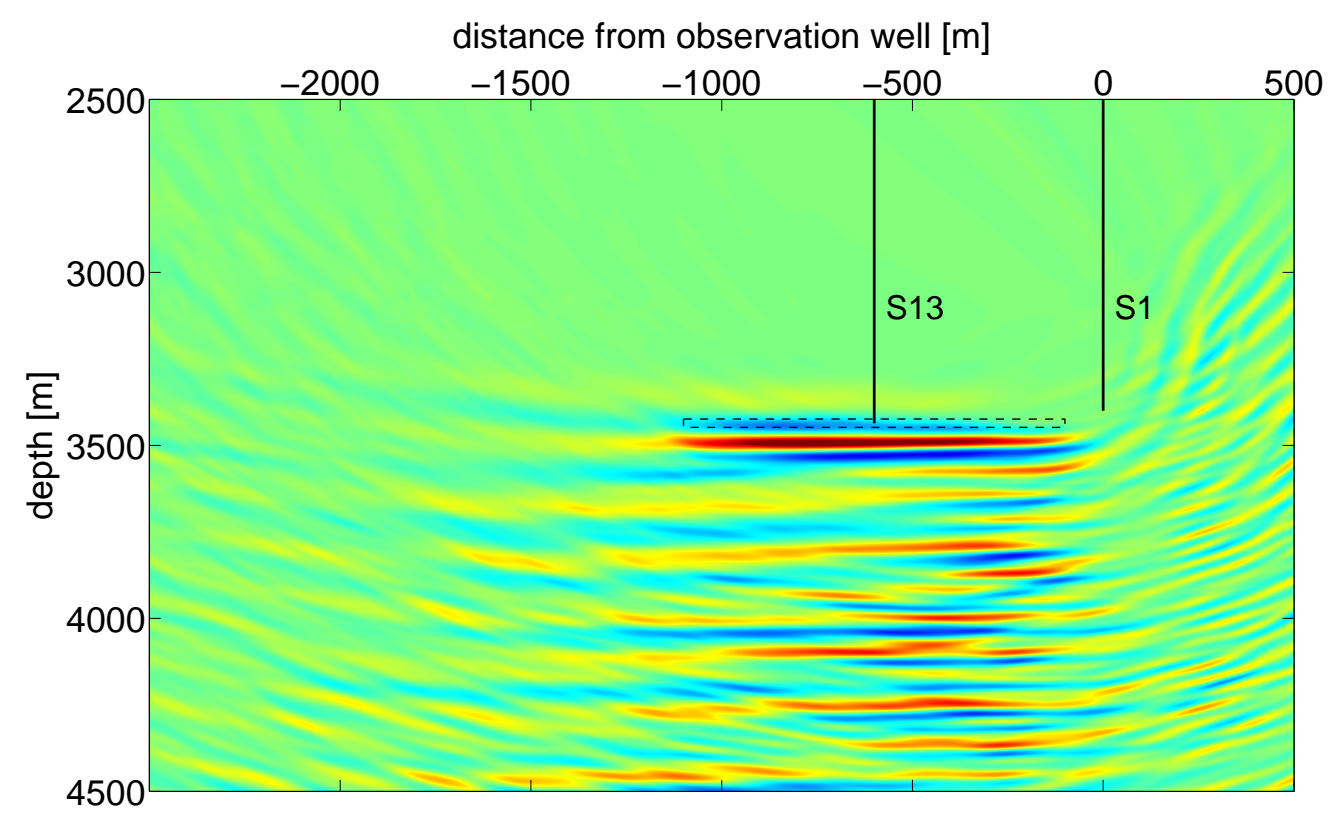

Figure 5.5: Result of standard Kirchhoff migration. It images the amplitude difference very well but artefacts caused by deeper reflections and multiples cannot be separated from $\mathrm{CO}_{2}$ accumulations below the reservoir.

\subsubsection{Fresnel-Volume migration}

In complex subsurface structures standard Kirchhoff migration often fails. Here Fresnel-Volume migration gives a clearer image of the spatial distribution of reflectors (Buske et al., 2009; Sick, 2005). This method was applied to the same data used in standard Kirchhoff migration, but in order to suppress the effects seen in figure 5.5, the traces were cut 3 period lengths after the onset of the first reflection (black dashed line in figure 5.3). This helps to give a clearer image and to reduce computing time, but it is not an appropriate method in an environment where the distribution of the $\mathrm{CO}_{2}$ in the subsurface is unknown and several $\mathrm{CO}_{2}$ layers may occur. The result is shown in figure 5.6.

Fresnel-Volume migration shows no significant improvements of the main reflector for this very simple model. Like the result from standard Kirchhoff migration it correlates very well with the $\mathrm{CO}_{2}$ saturated zone und is only slightly shifted to 


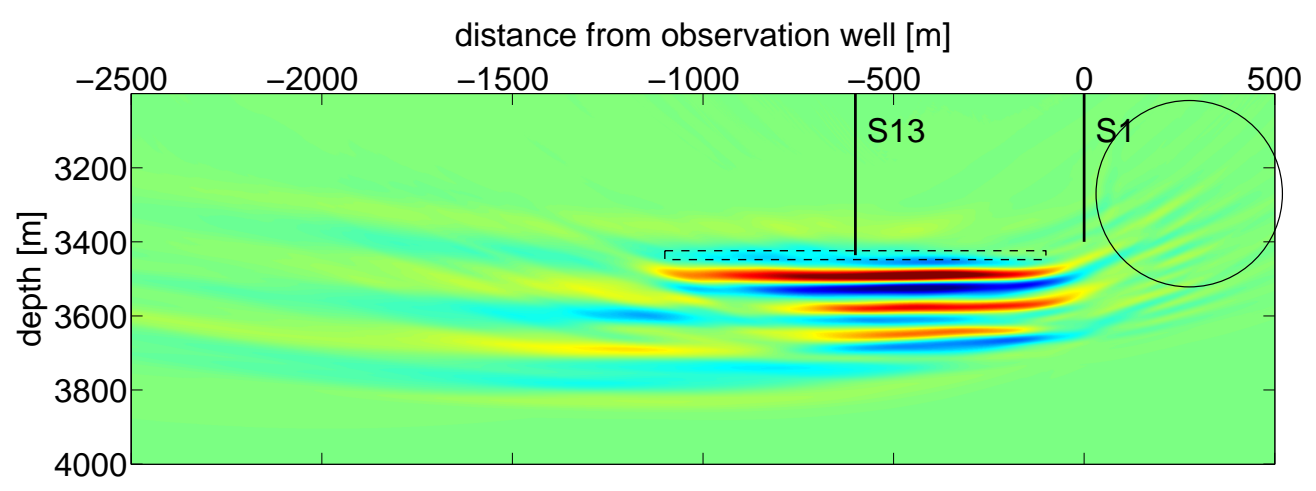

Figure 5.6: Result of Fresnel-Volume migration.

greater depth by approximately $30 \mathrm{~m}$. From the time restriction of the traces the great amount of reflections below the main reflector is suppressed. In comparison to figure 5.5 a significant improvement can be seen in the marked region (circle) to the right of the observation well S1. The previously diffuse smeared out energy (fig. 5.5) is now focused in the main reflectors (fig. 5.6).

\subsection{Noise}

Figure 5.7 a) shows the result of Kirchhoff migration applied to data with a $\mathrm{S} / \mathrm{N}$ ratio of 20. Here again the full $4 \mathrm{~s}$ traces of the receivers between $2200 \mathrm{~m}$ and $3300 \mathrm{~m}$ for 11 source positions were used. The localization of the $\mathrm{CO}_{2}$-cloud works still very well, even though the amplitude changes in the difference sections are nearly completely overlaid by noise and cannot be identified (fig. 4.5 e).

However, this strong random noise corrupts the estimation of the slowness leading to a slightly worse migration result. It is shown in figure $5.7 \mathrm{~b}$ ). Here, the same data as in 5.7 a) were migrated but with Fresnel-Volume migration. The matching of the velocity changes in this case are slightly worse and more energy is smeared outside of the reservoir zone.

\subsection{Shift}

The shift in receiver positions between baseline and repeat cause amplitude changes in the whole up-going wavefield (fig. 4.6 a). The migration of difference sections from 11 source positions and receivers in a depth of $2200 \mathrm{~m}$ to $3300 \mathrm{~m}$, each shifted by $1 \mathrm{~m}$, images the regions of impedance changes in the models quite well (fig. 5.8). It only shows the slight shift towards greater depth that was also obtained in the migration tests above and an incorrect extension of the $\mathrm{CO}_{2}$-cloud towards the 
a)

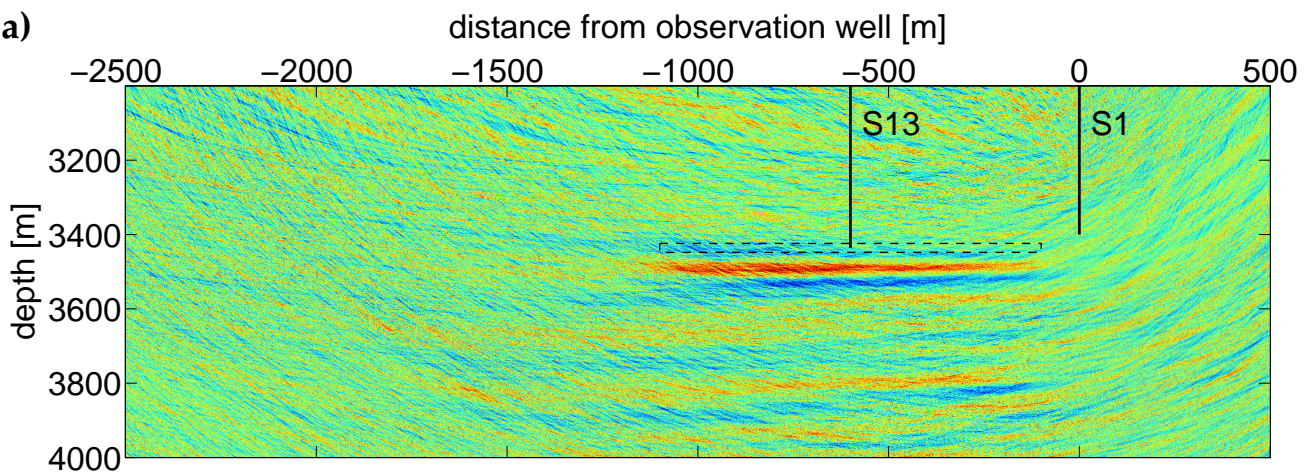

b)

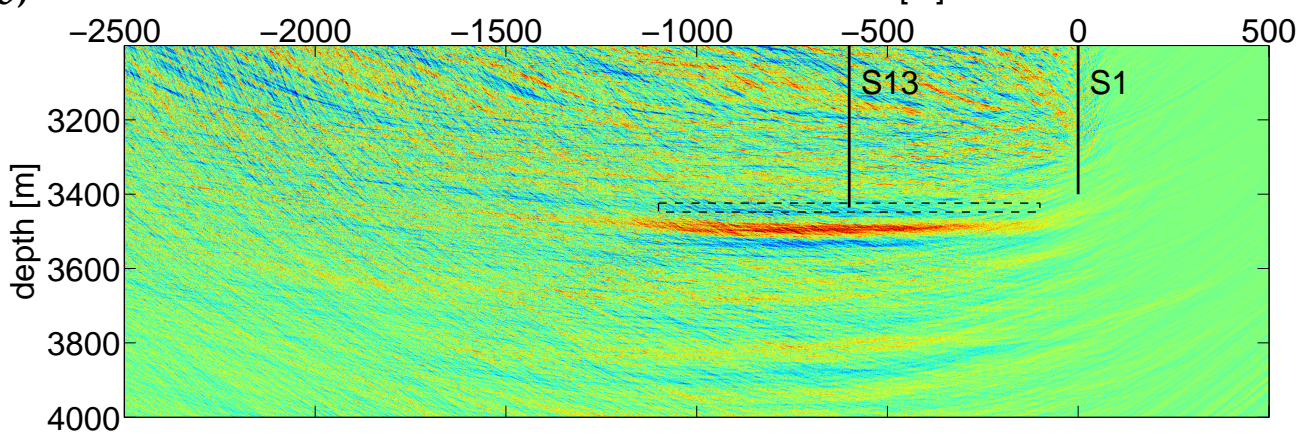

Figure 5.7: Kirchhoff migrated (a) and Fresnel-Volume migrated image (b) of data with a $\mathrm{S} / \mathrm{N}$-ratio of 20 .

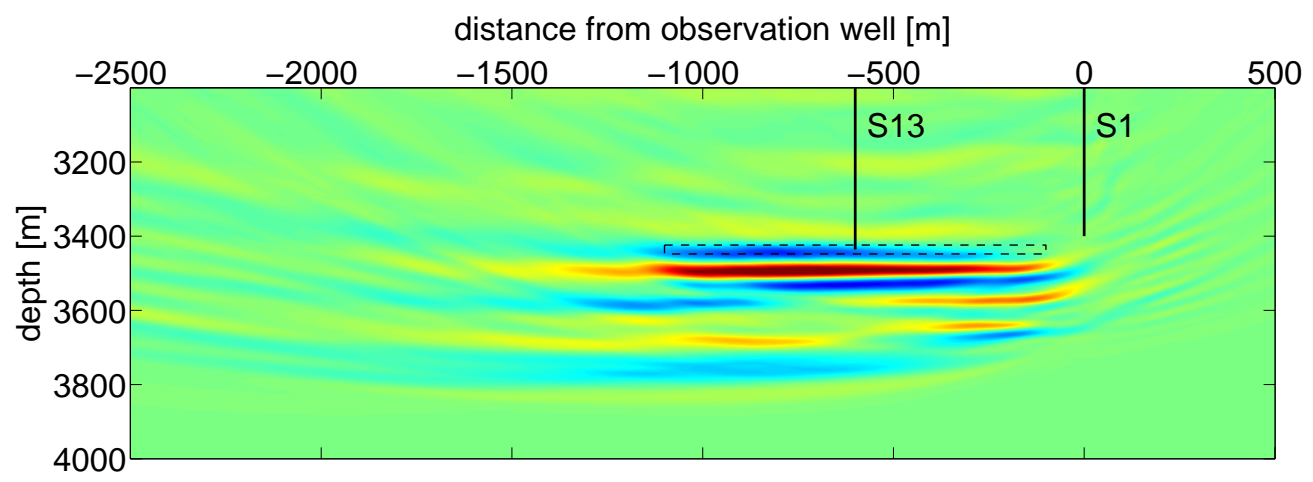

Figure 5.8: Fresnel-Volume migration of data with a shift in receiver positions between baseline and repeat of $1 \mathrm{~m}$. 
left of about $200 \mathrm{~m}$. The amplitude effects prior to the direct reflection from the reservoir, which were sometimes strong, did not sum up constructively and result in only weak artefacts in the area above the reservoir. This shows that shifts, even though significant in the difference section, do not cause a serious problem after migration.

\subsection{Source density}

Finally, it is very important for the experiment setup to find an optimum source density, i.e. optimum distance between two adjacent source positions. A number that is too small would not result in an optimum localization of the velocity changes, while a number that is too large would be uneconomic. Figure 5.9 shows FresnelVolume migration images with source positions every $1000 \mathrm{~m}$ (a), every $500 \mathrm{~m}$ (b) and every $250 \mathrm{~m}$ (c).

Sources which are positioned every $1000 \mathrm{~m}$ can not adequately locate the edges of the $\mathrm{CO}_{2}$ accumulation and smears them over. When using a source distance of $500 \mathrm{~m}$, the far edge is imaged very well, but the edge near to the observation well is still smeared. This is improved when using 22 source positions with an offset of $250 \mathrm{~m}$. With this configuration the coverage of the reservoir with reflection points is very dense.

Especially in near and far source-receiver offsets the use of a higher source density is recommended to achieve a high enough fold. A further decrease of the sourcesource offset does not result in a significant improvement.

\subsection{Conclusion}

The migration tests showed that difference sections can be used to locate the origin of amplitude changes. For the used experiment design with 111 receivers in a depth of $2200 \mathrm{~m}$ to $3300 \mathrm{~m}$ and 11 source positions with $500 \mathrm{~m}$ to $5500 \mathrm{~m}$ offset, the images show very good agreement between the lateral extent of the main reflector and the implemented impedance changes in nearly all cases. The reflectors were only slightly shifted below the lower boundary of the reservoir layer. Kirchhoff migration tests on the original, i.e. unsmoothed, velocity model identify the origin of these shifts in the not perfect though smoothed velocity model.

Further tests showed that disturbances in the data like white noise down to a S/N-ratio of about 20 and time shifts of less than $1 \mathrm{~m}$ do not cause significant problems when migrating a number of source positions, though random noise and artefacts resulting from shifts do not interfere constructively. The same happened 
to artefacts resulting from the incorrect repeat of receiver positions. Apart from a slight extension of the receiver averted boundary, they nearly completely sum out and a clear image of the velocity changed region remains.

One difficulty in the interpretation of migrated images results from artefacts that are inserted into the migration image by reflections from interfaces below the reservoir and multiples that traveled through the $\mathrm{CO}_{2}$ zone in the reservoir. They also insert amplitude changes in the seismic wavefield and are typically migrated in greater depth. A differentiation of these multiples from $\mathrm{CO}_{2}$-accumulations is only possible when differences in the wavefield are examined accurately.

a)

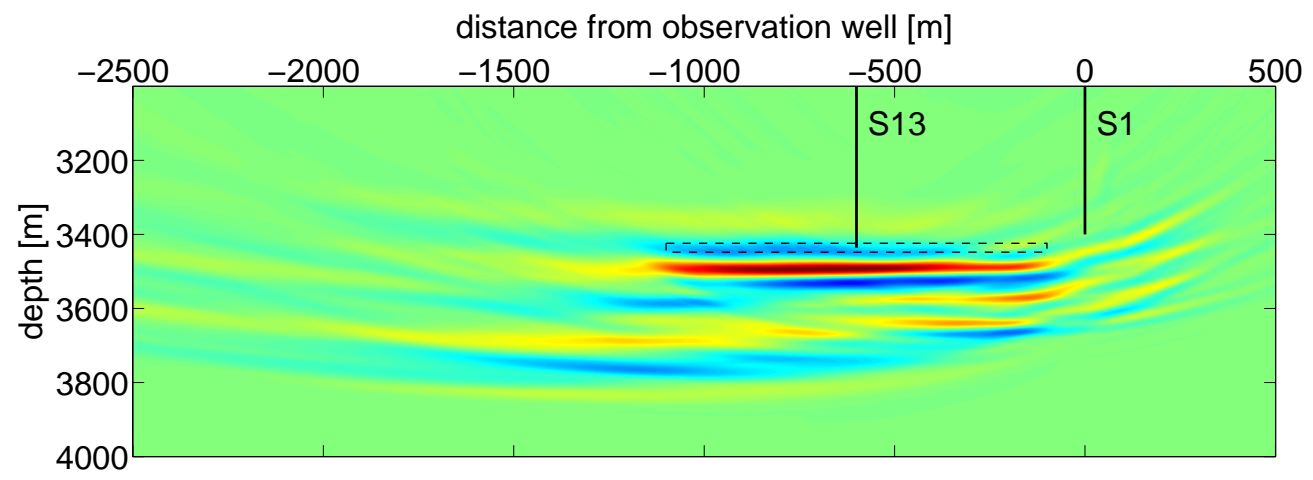

b)

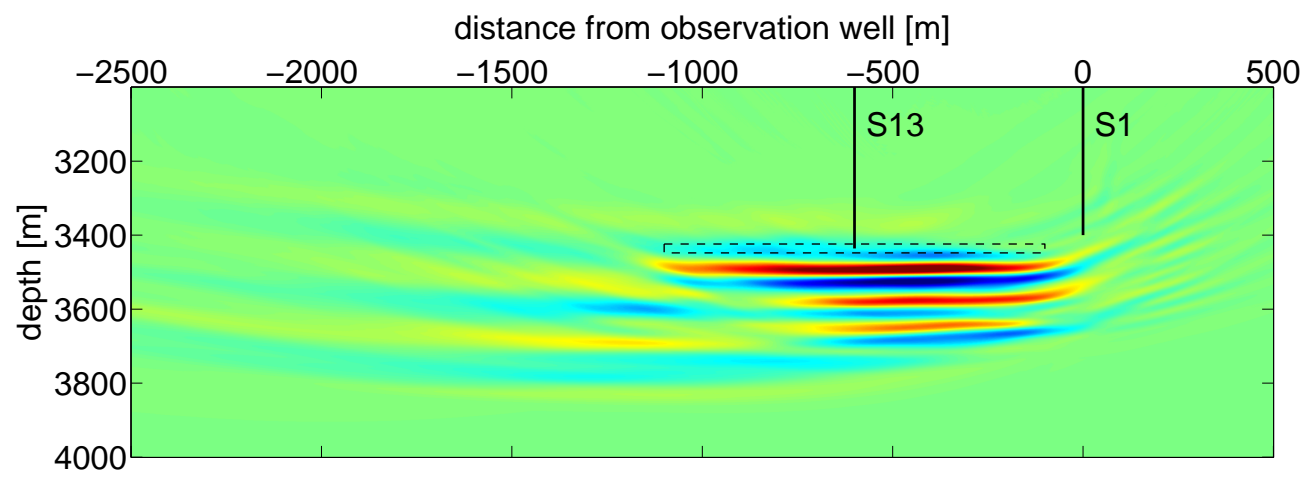

c)

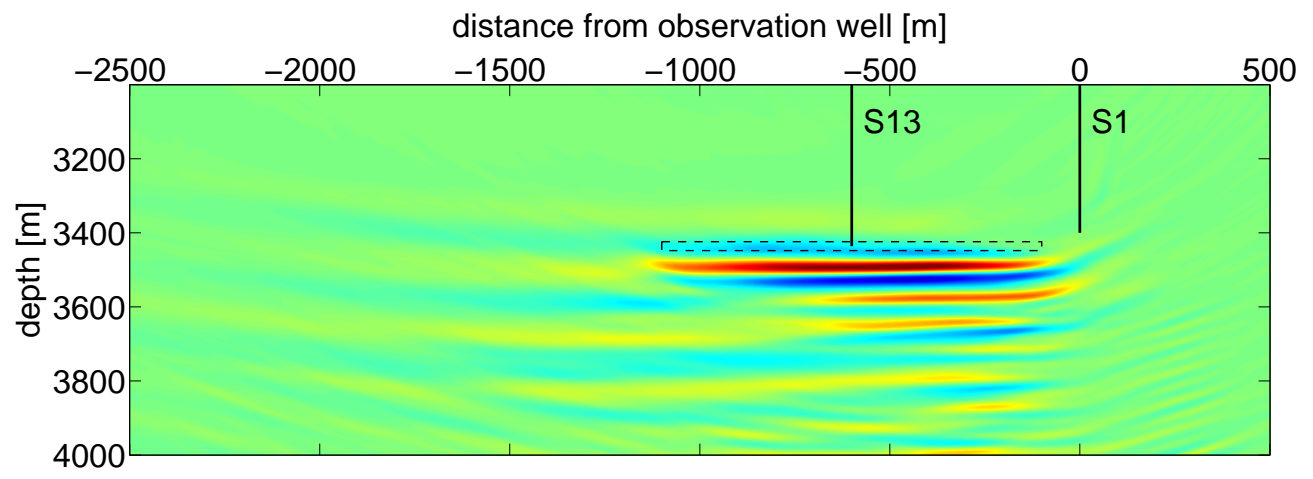

Figure 5.9: Fresnel-Volume migration of an inter-source distance of $1000 \mathrm{~m}$ (a), $500 \mathrm{~m}$ (b) and $250 m(\mathrm{c})$. 
In our models Fresnel-Volume migration provided only a slight increase in image quality. This is due to the fact that standard Kirchhoff migration works very well for horizontally layered media. When working with models or real data, where stratigraphy will be more complicated, the use of Fresnel-Volume migration is recommended.

For the experiment design it was found that a source-source offset of $250 \mathrm{~m}$ is most effective. 



\section{Chapter 6}

\section{Coda wave interferometry}

Beside changes in the reflection amplitude, $\mathrm{CO}_{2}$ induced changes in the velocity will cause time shifts. These time shifts are larger for waves that travel the changed region not just once but several times. Those multiples occur in the coda.

Snieder et al. (2002) inserted a method, where small time shifts in the coda waves are used to detect small temporal changes in a medium. The method is called "coda wave interferometry" (CWI). The time shifts are determined by a time windowed cross-correlation.

After explaining the theory of coda wave interferometry, some tests were carried out to explain the importance of the parameters used in this method. Afterwards CWI was applied to the Altmark models to estimate the dependencies on

- magnitude of changes in rock properties,

- propagation of the $\mathrm{CO}_{2}$ front,

- source-receiver offset.

Furthermore the sensibility to white noise and to shifts in receiver positions was tested.

\subsection{Theory}

The seismic wavefield in a strongly scattering medium, excited by a seismic source, can be given as the sum of waves $A(t)$ travelling along all possible trajectories $\mathrm{T}$

$$
u_{u}(t)=\sum_{T} A_{T}(t)
$$

$A_{T}(t)$ is the wave travelling along the specific trajectory $\mathrm{T}$. If the velocity of the medium changes with time, this will predominantly result in a change in the arrival 
times $t_{T}$ of the waves travelling along the trajectory. The perturbed wave is then given by

$$
u_{p}(t)=\sum_{T} A_{T}\left(t-t_{T}\right)
$$

The time-shifted cross-correlation over a time window of the unperturbed and perturbed wavefield gives the time-windowed correlation coefficient $\mathrm{R}\left(t_{s}\right)$

$$
R\left(t_{s}\right)=\frac{\int_{t-t_{w}}^{t+t_{w}} u_{u}\left(t^{\prime}\right) u_{p}\left(t^{\prime}+t_{s}\right) d t^{\prime}}{\sqrt{\int_{t-t_{w}}^{t+t_{w}} u_{u}^{2}\left(t^{\prime}\right) d t^{\prime} \int_{t-t_{w}}^{t+t_{w}} u_{p}^{2}\left(t^{\prime}\right) d t^{\prime}}} .
$$

When both wavefields are unperturbed $\left(u_{u}(t)=u_{p}(t)\right)$ the correlation-coefficient $R\left(t_{s}=0\right)=1$. When $u_{p}(t)$ is a time-shifted version of the unperturbed wavefield $\left(u_{u}(t)=u_{p}\left(t+t_{p}\right)\right)$ then $R\left(t_{s}\right)$ attains its maximum for a time shift $t_{s}=t_{p}=: t_{\text {max }}$. The time shift $t_{\max }$ is the average travel time difference of all waves travelling all possible trajectories and arriving in the analyzed time window.

This procedure is done for each time interval over a whole trace and gives the time $t_{\text {max }}(t)$ for which the correlation coefficient attains its maximum in dependence of the centre time $t$ of the time window. The time shift can be transformed into the relative velocity change by

$$
\frac{\delta v}{v}=-\frac{t_{\max }}{t}=-\frac{t_{p}}{t} .
$$

The mean velocity change in a certain depth can be calculated by averaging the relative velocity changes over the whole trace

$$
\left\langle\frac{\delta v}{v}\right\rangle=\frac{1}{N} \sum_{i=1}^{N}-\frac{t_{m a x, i}}{t_{i}}
$$

where $\mathrm{N}$ is the number of time samples per trace.

\subsection{Tests}

The characteristics of coda wave interferometry were tested on a model with velocity changes of $-6 \%$ (from $4500 \mathrm{~m} / \mathrm{s}$ to $4230 \mathrm{~m} / \mathrm{s}$ ) in $2400 \mathrm{~m}$ to $2845 \mathrm{~m}$ depth (fig. 6.1). It is a streched version of the model used by Zhou et al. (2010), which is based on a profile of the Marmousi model, with modified layer thickness. These conditions cause relatively large time shifts in the coda in the dimension of $3 / 4 T_{c}$, where the effect of parameters can be shown quite well. The source was a $25 \mathrm{~Hz}-\mathrm{Ricker}$ signal. The synthetic data were pre-processed as described in chapter 3.3.

From these tests we found that there are mainly two parameters that control the stability of the result, which are 
- the maximum time shift $t_{s}$

- the length of the time window $2 t_{w}$.

They were analyzed separately to find optimum parameters for further applications to the Altmark-model.

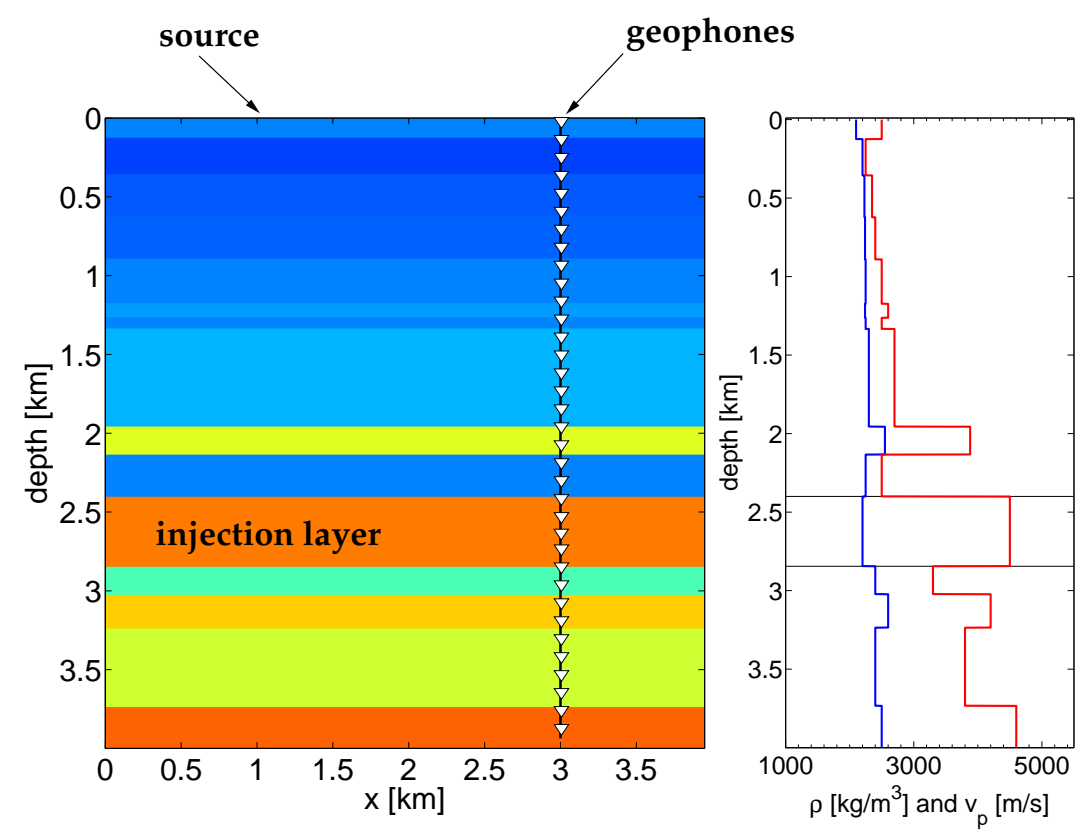

Figure 6.1: Velocity model used for the interferometry tests.

\subsubsection{Maximum time shift $t_{s, \max }$}

Under the assumption, that the frequency does not change too much over the measured time range, formula 6.3 will always result in time shifts that correlates the signal from the analysed time window of the unperturbed wavefield to the strongest event in the perturbed wavefield (in most cases the first break) if the centre time of the moving time window $t+t_{s}$ can take all values from the beginning to the end of the trace. The estimation of the relative velocity change becomes better, if we force the calculation to smaller time shifts by restricting $t_{s}$.

This restriction of $t_{s}$ is very important especially for structural conditions that generate large time shifts in the dimension of about half a period length or more, as is the case in the enlarged model in fig. 6.1. Then optimum results are found, when $t_{s}$ is restricted to the maximum time shift that occurs in the section.

To find the optimum $t_{s, \max }$ one has to look at the calculated time shifts $t_{\max }$. Figure 6.2 shows the maximum time shifts $t_{\max }$ for different $t_{s, \max }$ in trace 240 at 

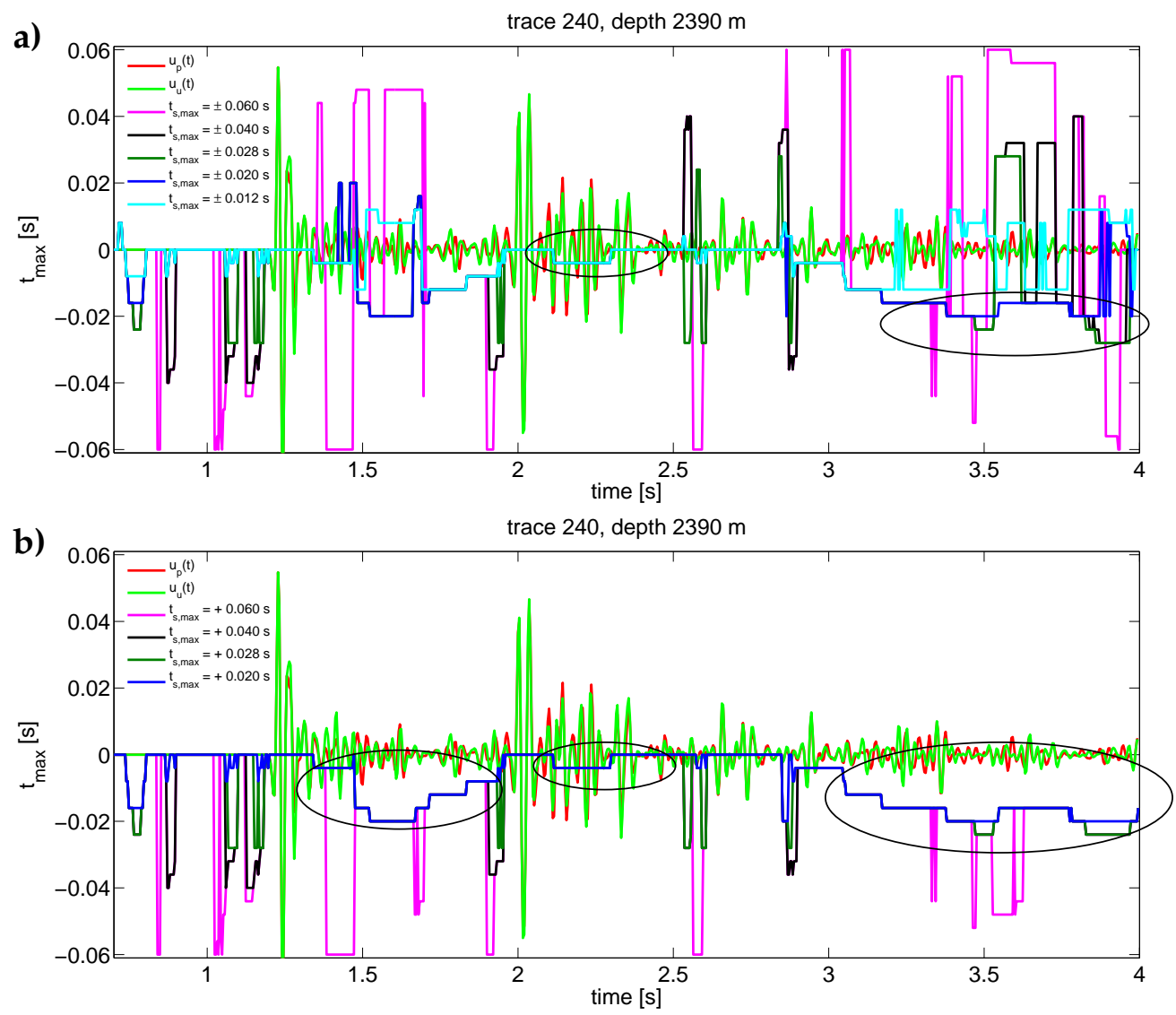

Figure 6.2: Calculated maximum time shifts $t_{s, \max }$ for different restrictions of $t_{s}$. In b) $t_{s, \max }$ is confined to positive values.

$2390 \mathrm{~m}$ depth, i.e. just above the velocity-changed layer. Beside strong fluctuations in some parts, there are always periods, where the calculated time shift $t_{\max }$ is identical for all (or at least most) curves (see marked regions in figure 6.2 a). In these regions we can expect, that the calculated time shifts $t_{\max }$ represent the "real" time shift $t_{p}$ between $u_{u}(t)$ and $u_{p}(t)$, i.e. $t_{\max }=t_{p}$. Here especially the curves with $0.02 s \leq t_{s, \max } \leq 0.04 s$ show very good agreement. For $t_{s, \max }> \pm 0.04 s$ the larger range causes the problem that the largest correlation coefficient leads not always to the shifted wavelet and so the "real" time shift, if a wavelet with a larger amplitude lies within the range for $t_{s}$. Of course this probability increases with increasing time range for $t_{s}$. For $t_{s, \max }<0.02 \mathrm{~s}$ the time range is too small, as the "real" time shifts in the later part of the seismograms, where we have multiple reflections, are at about $0.028 \mathrm{~s}$.

If velocities in the subsurface decrease, which is the case for the injection of $\mathrm{CO}_{2}$ (fig. 7.3), the perturbed waves, i.e. the waves that travel once or multiple times in the changed region, are expected to arrive later than the unperturbed wave. Because 


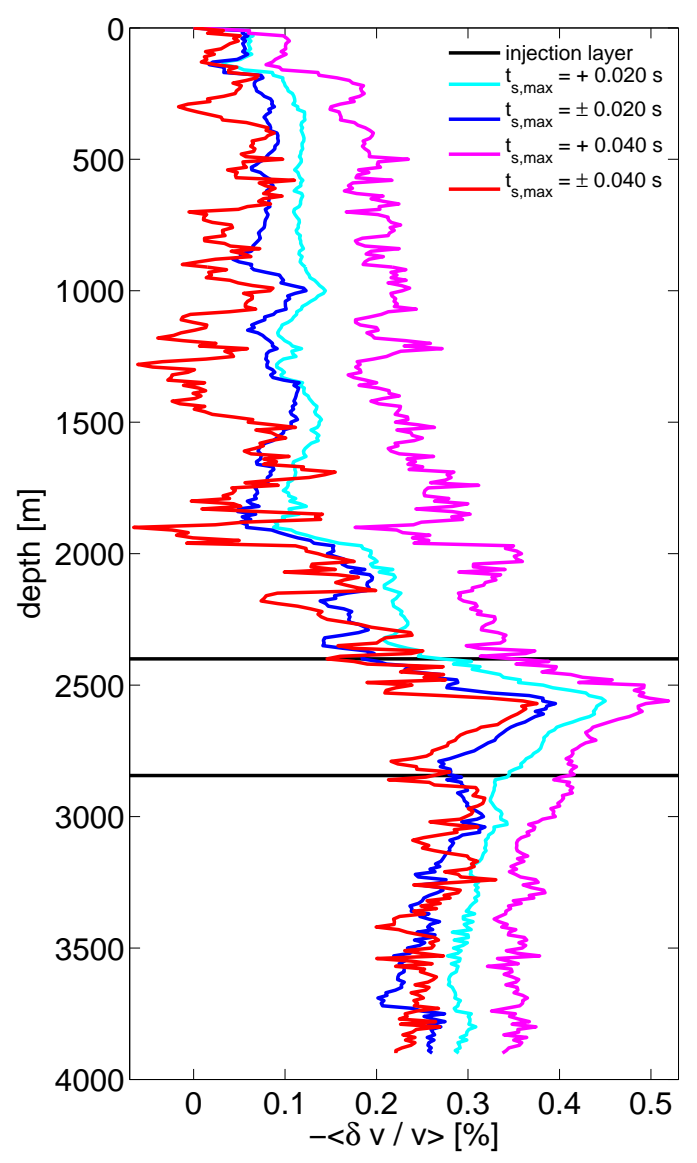

Figure 6.3: Mean velocity change for positive and negative and only positive allowed $t_{s, \max }$.

of this consideration $t_{s}$ was allowed just to take positive values.

Only positive allowed $t_{s}$ result in a very nice trend for all three curves (fig. 6.2 b) with fewer fluctuations than in figure $6.2 \mathrm{a}$ ), but also the effect of the statistic correction of those fluctuations gets lost. This arises in a shift of the relative velocity change $\langle\delta v / v\rangle$ towards higher values (positive and negative fluctuations usually sum out) and we achieve no prominent enhancement (fig. 6.3).

However when large time shifts of about $0.5 T_{c}$ or more occur, a strict confinement of $t_{s, \max }$ is necessary in order to reduce miscalculations due to positive amplification with neighbouring wave trains. For smaller time shifts the restriction can be more generous, but should be less than $0.5 T_{c}$. This general rule of course also depends on the width of the power spectrum and will not give satisfying results when lots of higher and lower frequencies occur. 


\subsubsection{Length of the time window $2 t_{w}$}

The temporal window length $2 t_{w}$ can be very important for the estimation of the velocity change with coda wave interferometry. Figure 6.4 shows the calculated velocity change for different time windows. It shows that time windows of less than about 3 wave lengths $(0.12 \mathrm{~s})$ are too small. The calculation of $t_{\max }$ gives lots of fluctuations for $2 t_{w}=0.06 \mathrm{~s}$ (fig. $6.5 \mathrm{a}$ ) that sum out during the final averaging and mean velocity changes are flucuating throughout the whole depth and are not clearly indicating the depth, where the velocity changes occurred (fig. 6.4 blue curve).

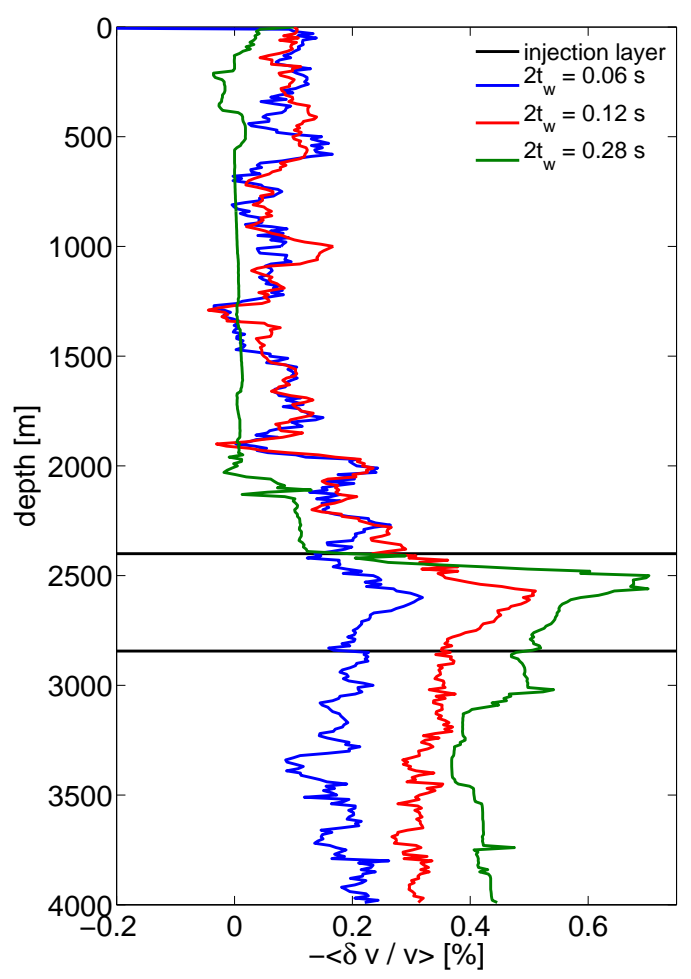

Figure 6.4: Mean velocity change for different time windows $\left(2 t_{w}\right)$.

If the time window is enlarged, the maximum of the mean velocity change increases at the depth of the injection layer while simultaneously the changes above $2400 \mathrm{~m}$ do not change significantly (fig. 6.4 red curve).

Figure $6.5 \mathrm{c}$ ) to d) show that with increasing time window the calculated time shifts $t_{\max }$ of up-going waves above the reservoir layer are more and more smoothed out until finally for $2 t_{w}=1.2 \mathrm{~s}\left(30 T_{c}\right)$ only the injection layer itself and the region below shows relevant time shifts (fig. $6.5 \mathrm{~d}$ ). Even though lots of information about maximum time shifts and their occurence in the wavefield gets lost, large 
a)

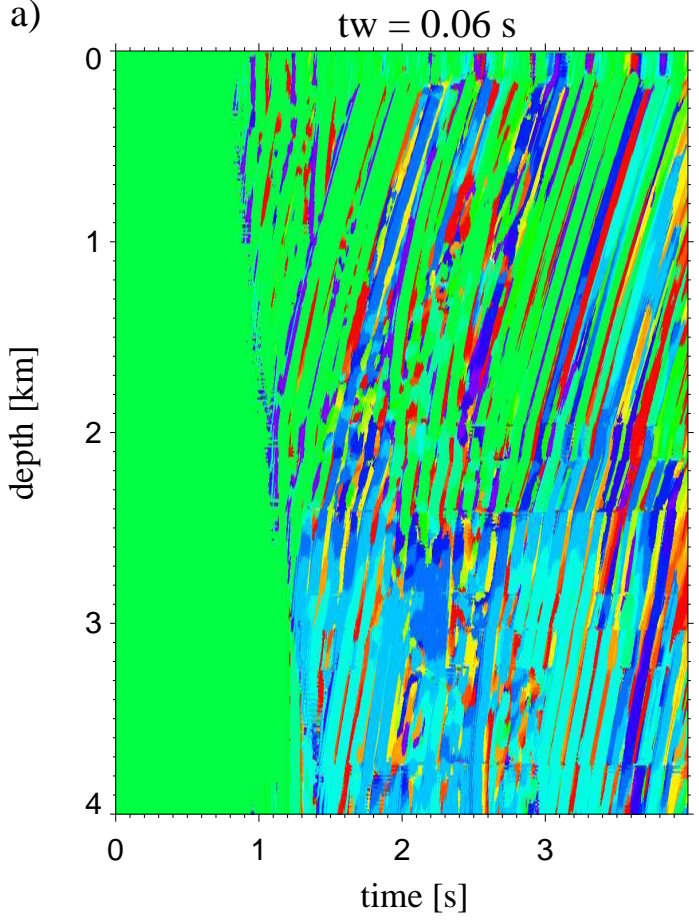

c)

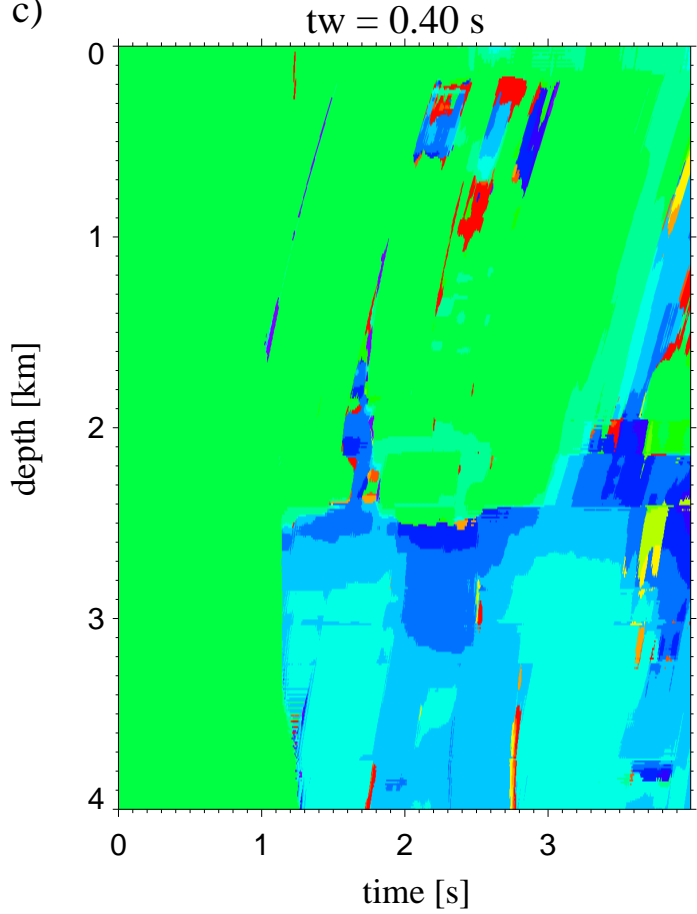

b)

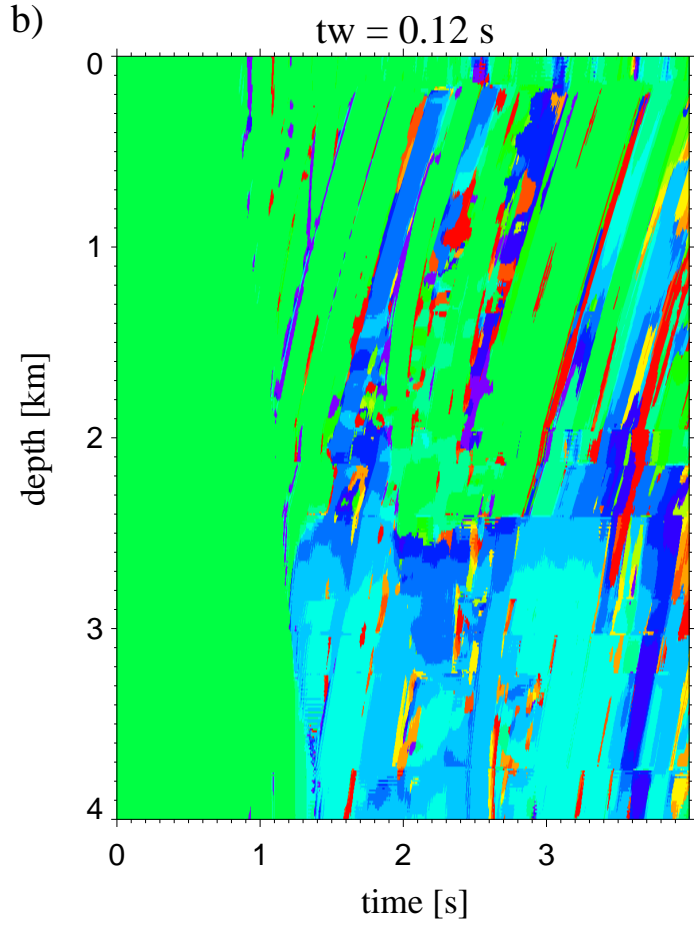

d)

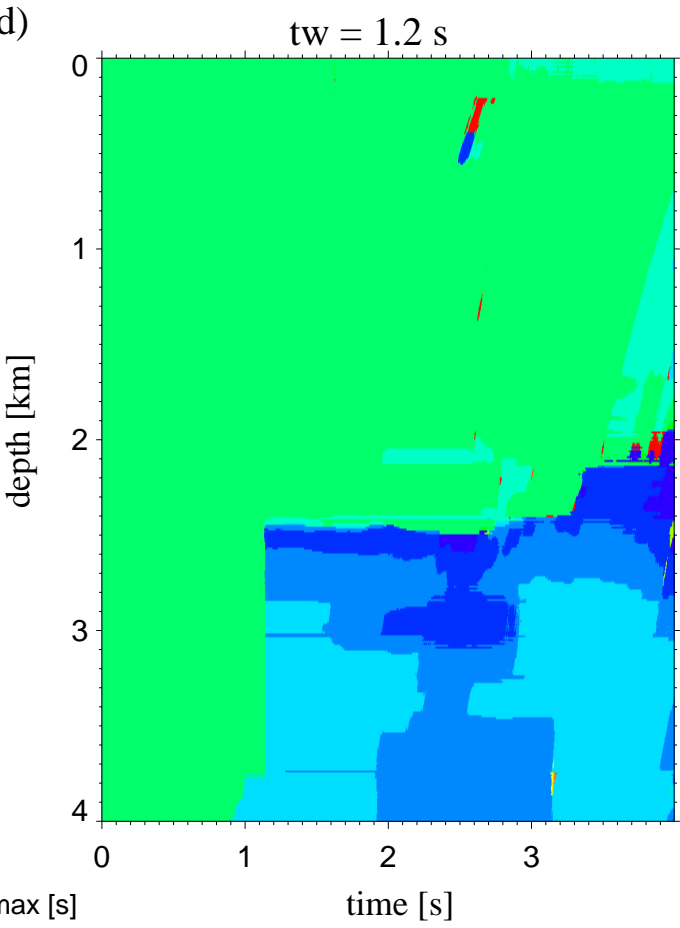

$\begin{array}{lllll}-0.02 & -0.01 & 0.00 & 0.01 & 0.02\end{array}$

Figure 6.5: Time shifts calculated with time windows of $2 / 3 T_{c}$ (a), $3 T_{c}$ (b), $10 T_{c}$ (b), $30 T_{c}$ (d). 
time windows better calculate the depth range and the size of the mean velocity changes (fig. 6.4 green curve). For models with small to moderate velocity changes, the importance of $2 t_{w}$ decreases and large time windows do not improve the results significantly.

Finally we can state that a time window of 3 to 4 wave periods as it is recommended in Zhou et al. (2010) is adequate for model conditions causing small time shifts. However time windows below $5 T_{c}$ seem to be too small when large time shifts of more than about $0.5 T_{c}$ occur and much larger windows of about 7 up to 30 wave periods will lead to better results (fig. 6.4).

\subsection{Application to the Altmark-model}

After these tests the influence of the parameters is better understood and coda wave interferometry can be applied to the Altmark data. Here very small time shifts of a few time samples are expected (sample rate $\Delta t=0.2 \mathrm{~ms}=1 / 200 T_{c}$ ). The time shifts were restricted to $1 / 10$ of the centre period length $\left(t_{s}= \pm 4 \mathrm{~ms}\right)$ and a time window of $7 T_{c}\left(2 t_{w}=280 \mathrm{~ms}\right)$ was used.

In order to test if coda wave interferometry can be used to qualitatively and quantitatively estimate the velocity changes in the subsurface, a number of tests were performed, seeing the influence of different factors like velocity change in the subsurface, extension of these changes as well as source-receiver offsets.

$0.01 \%$ was set as a lower boundary for the detectability of those mean velocity changes in these tests. This threshold was indicated by Poupinet et al. (1984), who used a similar technique to monitor velocity changes caused by earthquake conditioned changes in stress and in the water table. Finally this threshold was verified by examine the influence of noise and shifts in receiver positions.

\subsubsection{Velocity changes}

In a first step the effect of different amounts of velocity changes in the subsurface on mean velocity changes was tested. Again the Altmark models presented in figure 3.3 were used and the velocity in the reservoir layer was changed by differing amounts within a radius of $1000 \mathrm{~m}$ around the injection well (fig. $6.7 \mathrm{a}$ ). The radius is based on the estimated $\mathrm{CO}_{2}$ propagation in the reservoir after 2 years. Figure 6.6 shows mean velocity changes calculated on models with $\mathrm{P}$-wave velocity changes of $4 \%, 2 \%$ and $1 \%$ and the relative S-wave velocity changes. All three models show principally the same trend for P- and S-waves, respectively, but with different maxima. They show a permanent increase with depth, accelerating when nearing 
the reservoir and reaching its maximum right at the top of the reservoir. The P-wave shows a broader maximum region with a plateau in the range from the top reservoir to about $300 \mathrm{~m}$ above it while the S-wave shows a sharp peak. The reservoir itself is indicated by a sharp drop of mean velocity changes. For large velocity changes $\left(\Delta v_{p}=\Delta v_{s}=-4 \%\right)$, the maximum of mean velocity changes can be identified inside the reservoir, an effect that was already obtained in the test models.

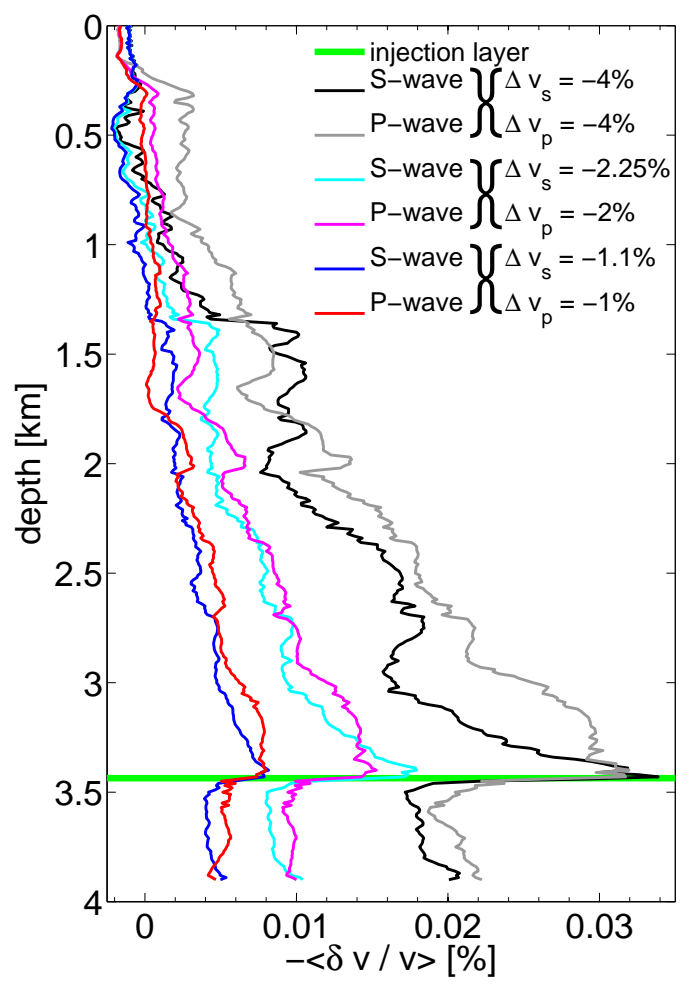

Figure 6.6: Calculated mean velocity change for models with variable velocity changes in the reservoir.

In between, at about $1400 \mathrm{~m}$ depth, a sudden increase appears in mean S-wave velocity changes. It correlates very well with the high velocity zone in the model (see fig. 3.3 and 5.4) and might arise from strong reflections and conversions of the up-going waves. Also for P-waves a peak at about $2000 \mathrm{~m}$ depth appears, correlating with the second high velocity zone in the model.

The curves depict the doubling in P-wave velocity change very well by almost a doubling of the maxima with $-0.008 \%$ for $\Delta v_{p}=-1 \%,-0.015 \%$ for $\Delta v_{p}=-2 \%$ and $-0.032 \%$ for $\Delta v_{p}=-4 \%$. For the model with $\Delta v_{p}=-2 \%$ and $\Delta v_{s}=-2.25 \%$ the differences in $\mathrm{P}$ - and $\mathrm{S}$-wave velocity changes, result in different maxima for mean $\mathrm{P}$ - and S-wave velocity changes, while for the model with equal changes for $\mathrm{P}$ - and 
S-waves, also the calculated mean velocity changes show equal maximum values. However, this does not hold for the $-1 \%$ model.

Altogether, the calculated mean velocity changes can reproduce the ratio of implemented velocity changes for P- and S-waves as well as for different values of either of the components. The location of the reservoir is indicated by a maximum at its top or inside the reservoir layer if produced time shifts are large enough, followed by a sharp drop. Strong velocity contrasts can cause jumps in the curves as time shifted wavelets can be trapped. So mean velocity changes have to be analysed carefully to avoid misinterpretations.

\subsubsection{Propagation}

To see if a quantitative interpretation of the results shown in the previous chapter is possible, other influences that can effect the size of mean velocity changes have to be tested. Therefore mean velocity changes for different propagations of the $\mathrm{CO}_{2}$ front were calculated, which appears to be a crucial parameter for coda wave interferometry. In the previous tests it was assumed that velocity changes always occur in the whole layer or at least in a large part of the reservoir. This is not a realistic case for the $\mathrm{CO}_{2}$-injection. Beside the expansion it is also of great importance whether the $\mathrm{CO}_{2}$ surrounds the observation well or a $\mathrm{CO}_{2}$-free region exists around the well. This is easily the case if the injection well is not the same as the monitoring well, which is the situation in the CLEAN-project. These parameters are examined in figure 6.7. It shows mean velocity changes of three different models in d) and the corresponding velocity changed regions in the models (a-c). In all three cases the velocity was decreased by $\Delta v_{p}=-2 \%$ and $\Delta v_{s}=-2.25 \%$. The source was placed $2500 \mathrm{~m}$ away from the observation well.

Model A is the same model already analysed above (chap. 6.3.1). It has an expansion radius of $1000 \mathrm{~m}$ around the injection well, also enclosing the observation well (fig. $6.7 \mathrm{a}$ ). It is based on the estimated $\mathrm{CO}_{2}$ propagation in the reservoir after 2 years. Mean velocity changes calculated for S-waves (fig. 6.7 black curve) show a moderate increase starting from about $500 \mathrm{~m}$ depth until the increase gets steeper at about $3100 \mathrm{~m}$ and reaches its maximum of about $-0.018 \%$ right at the top of the reservoir. The reservoir itself is indicated by a sharp drop of mean velocity changes to $-0.008 \%$. Also for P-waves the increase gets steeper at about $2900 \mathrm{~m}$ and reaches a maximum of $-0.015 \%$ at top reservoir. The peak is broader than for S-waves. The reservoir is again indicated by a sharp drop of mean velocity changes.

However when changing the velocity in the hole reservoir layer, it only slightly increases the mean velocity change further. The maximum is reached when all up- 
a)
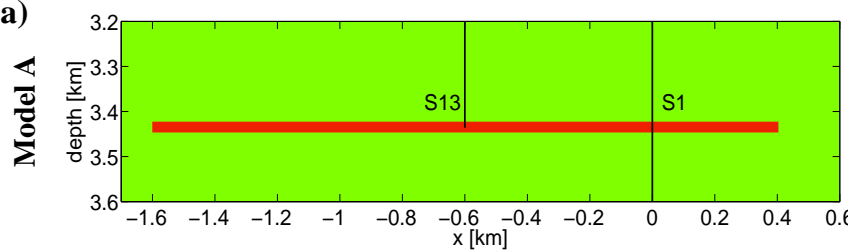

b)

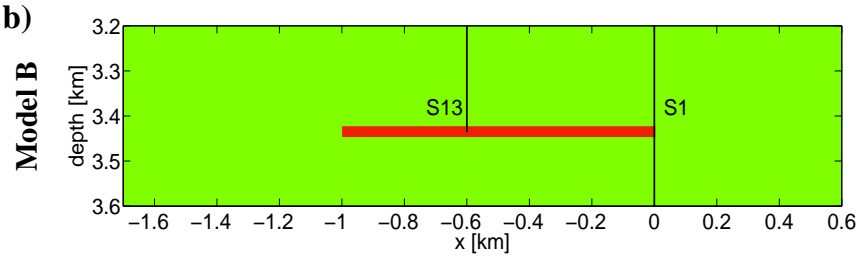

c)

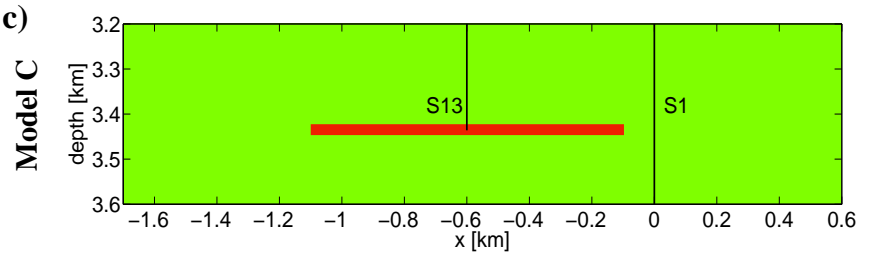

d)

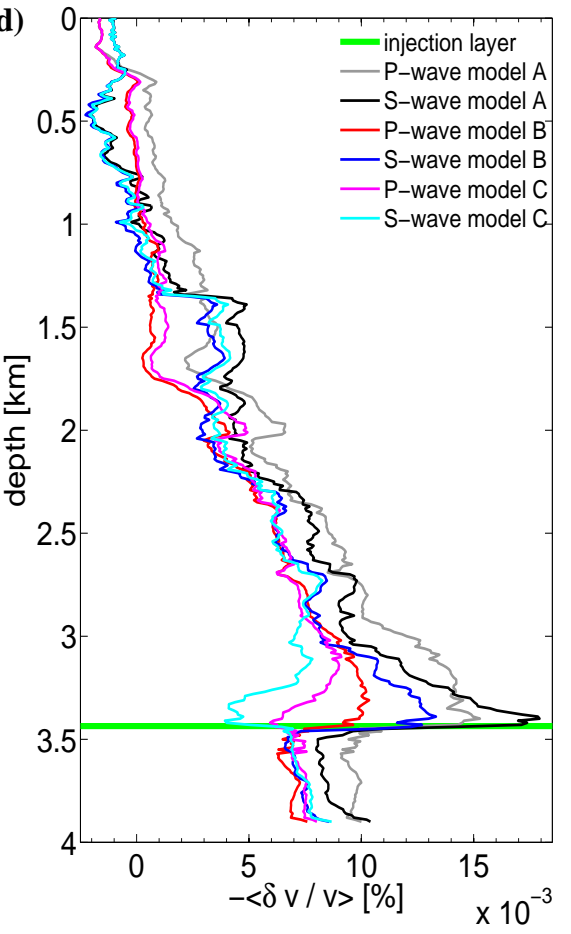

Figure 6.7: Difference in velocity models indicating the velocity changed zones (a)-(c) and corresponding mean velocity changes (d).

going waves reaching the receiver at the top of the reservoir had to pass the changed zone at least two times, once on its way down and the second time on its way up. For our geometry with a source-receiver offset of $2500 \mathrm{~m}$ and a limited depth of the model $(4950 \mathrm{~m})$ this is the case when about $2000 \mathrm{~m}$ around the observation well is $\mathrm{CO}_{2}$ saturated.

To further determine the effect of propagation, we assumed the $\mathrm{CO}_{2}$-cloud to have a diameter of $1000 \mathrm{~m}$ but still having a breakthrough at the observation well (model B, fig. $6.7 \mathrm{~b}$ ). The calculated mean velocity changes show a similar trend as for model A but with a less steep ascent above the reservoir and smaller amplitudes of about $-0.013 \%$ for S-waves and $-0.01 \%$ for P-waves (fig. $6.7 \mathrm{~d}$ ). The reservoir depth is still indicated by a steep descent.

Finally in model $\mathrm{C}$ the $\mathrm{CO}_{2}$ extended $500 \mathrm{~m}$ in both directions around the injection well. In this case the same amount of $\mathrm{CO}_{2}$ as in model $\mathrm{B}$ is inserted in the reservoir but a $\mathrm{CO}_{2}$-free gap exists between the $\mathrm{CO}_{2}$-front and the observation well (fig. 6.7 c). This model is an estimate of the $\mathrm{CO}_{2}$ propagation in the Altmark reservoir within 1 year. Mean velocity changes calculated for this model are nearly identical to model A and B in the upper $2800 \mathrm{~m}$. But then it shows an opposite 
trend and decreases when further approaching reservoir depth with a minimum at the top of the reservoir. Inside the reservoir layer it jumps back to higher values.

\subsubsection{Source-receiver offset}

Beside a direct variation of velocity changes in the subsurface and the extension of the changed zone, also the variation of the offset influences mean velocity changes.

Figure 6.8 shows mean velocity changes calculated for model A with P-wave velocity changes of $-2 \%$ and S-wave velocity changes of $-2.25 \%$ and a propagation of the $\mathrm{CO}_{2}$-front of $1000 \mathrm{~m}$ radius but for different source receiver offsets. The light blue and pink curve show mean P- and S-wave velocity changes, respectively, for an offset of $2500 \mathrm{~m}$. They were already analysed above and show maxima of $-0.015 \%$ (P-wave velocity) and $-0.018 \%$ (S-wave velocity). The blue and red curve in figure 6.8 show mean velocity changes for the same model, but with a source-receiver offset of $4000 \mathrm{~m}$ instead of $2500 \mathrm{~m}$. For this geometry the maximum mean S-wave velocity change above the reservoir increases slightly to about $0.0195 \%$ (blue), while for P-waves mean velocity changes decreased significantly to $0.0125 \%$ (red).

Additionally time shifts are better focused above the reservoir, an effect that was

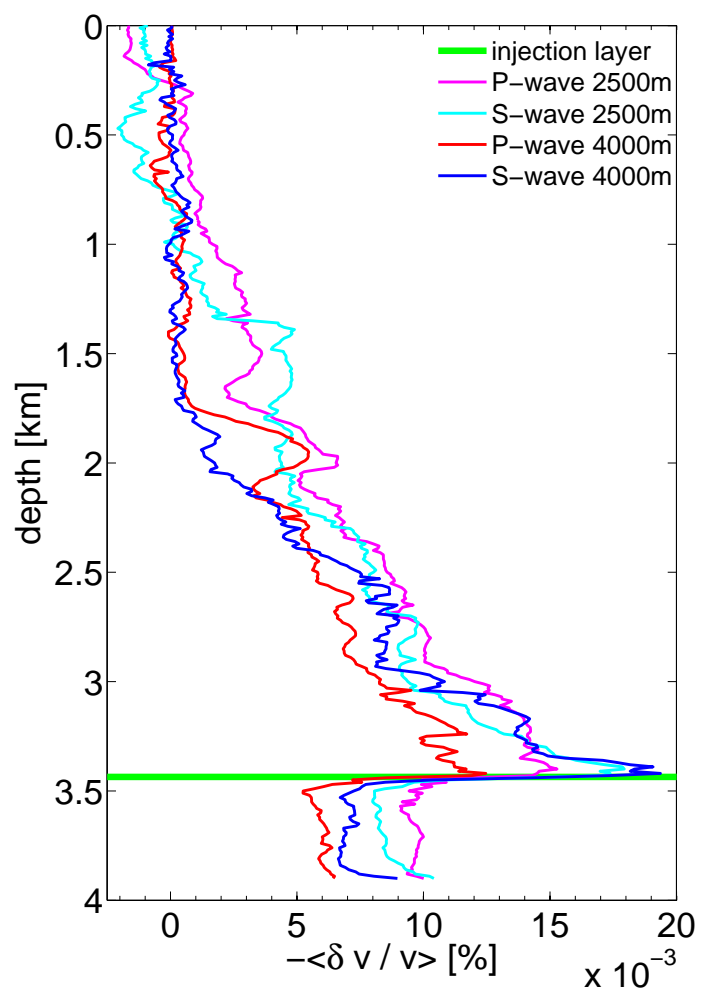

Figure 6.8: Mean velocity change calculated for models with different source-receiver offsets. 
already observed during the analysis of amplitude changes (fig. 4.4). For an offset of $4000 \mathrm{~m}$ they are approximately zero until about $1700 \mathrm{~m}$ depth in both components, while mean velocity changes start to increase much earlier at about $500 \mathrm{~m}$ depth for the data with an offset of $2500 \mathrm{~m}$. Then mean S-wave velocity changes show a steep ascent to its absolute maximum. However, below $1700 \mathrm{~m}$ mean P-wave velocity changes show nearly the same trend as for an offset of $2500 \mathrm{~m}$ but shifted by about $-0.0025 \%$.

\subsubsection{Leakage}

The tests above show that a quantitative analysis of velocity changes in the subsurface needs further efforts. But a prediction of the depth range is possible when changes are large enough and in the direct vicinity of the observation well. In a further test a leakage was implemented in model A with $\mathrm{P}$ - and S-wave velocity changes of $-4 \%$ at $2045 \mathrm{~m}$ depth. High changes like this are realistic, when formation water is present (Xue and Ohsumi, 2004). This leakage zone is very small with an extension of only $30 \mathrm{~m}$ and a thickness of $15 \mathrm{~m}$ (fig. 6.9 a). The corresponding

a)

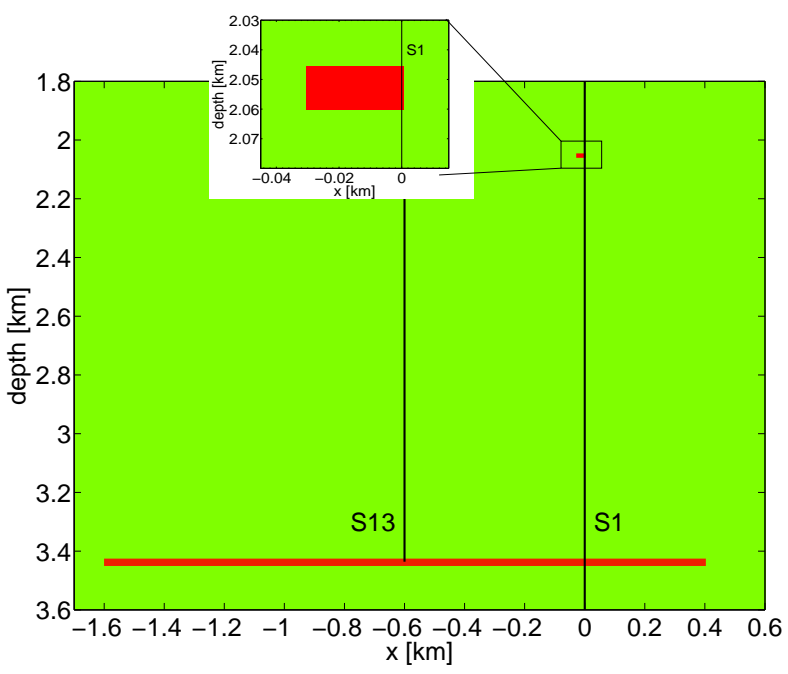

b)

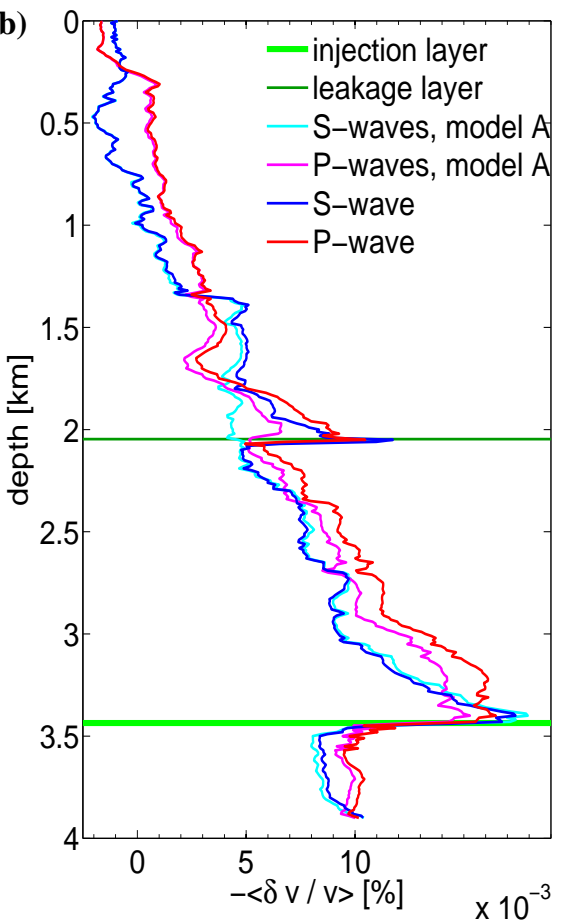

Figure 6.9: Model of a leakage case (a) and the calculated mean P- and S-wave velocity changes (b). For comparison mean velocity changes without leakage are also shown (pink and light blue). 
mean velocity changes in figure $6.9 \mathrm{~b}$ ) show the same trend as without the leakage, just with a peak of about $-0.012 \%$ for the S-wave and $-0.0095 \%$ for the P-wave, indicating the leakage zone. Below the leakage, mean S-wave velocity changes fall back to the values without leakage, while the P-wave changes stay slightly shifted.

\subsubsection{Noise}

Now the effect of noise on the method of coda wave interferometry is analysed. Therefore the data are disturbed with white noise (equal power spectrum) of different $\mathrm{S} / \mathrm{N}$-ratios as explained in chapter 3.3.3. Figure 6.10 shows the mean $\mathrm{S}$-wave velocity change for the leakage model in figure 6.9 a) (reservoir: $\Delta v_{p}=-2 \%$ and $\Delta v_{s}=$ $-2.25 \%$, leakage: $\Delta v_{p}=\Delta v_{s}=-4 \%$ ) disturbed with noise of $\mathrm{S} / \mathrm{N}$-ratios of 40 (a) and 20 (b). The red curves illustrates mean S-velocity changes of the leakage model without noise.

An S/N-ratio of 40 (fig. 6.10 a) poses no problem to the interpretation of mean velocity changes. The curve is very similar to the one without noise especially for
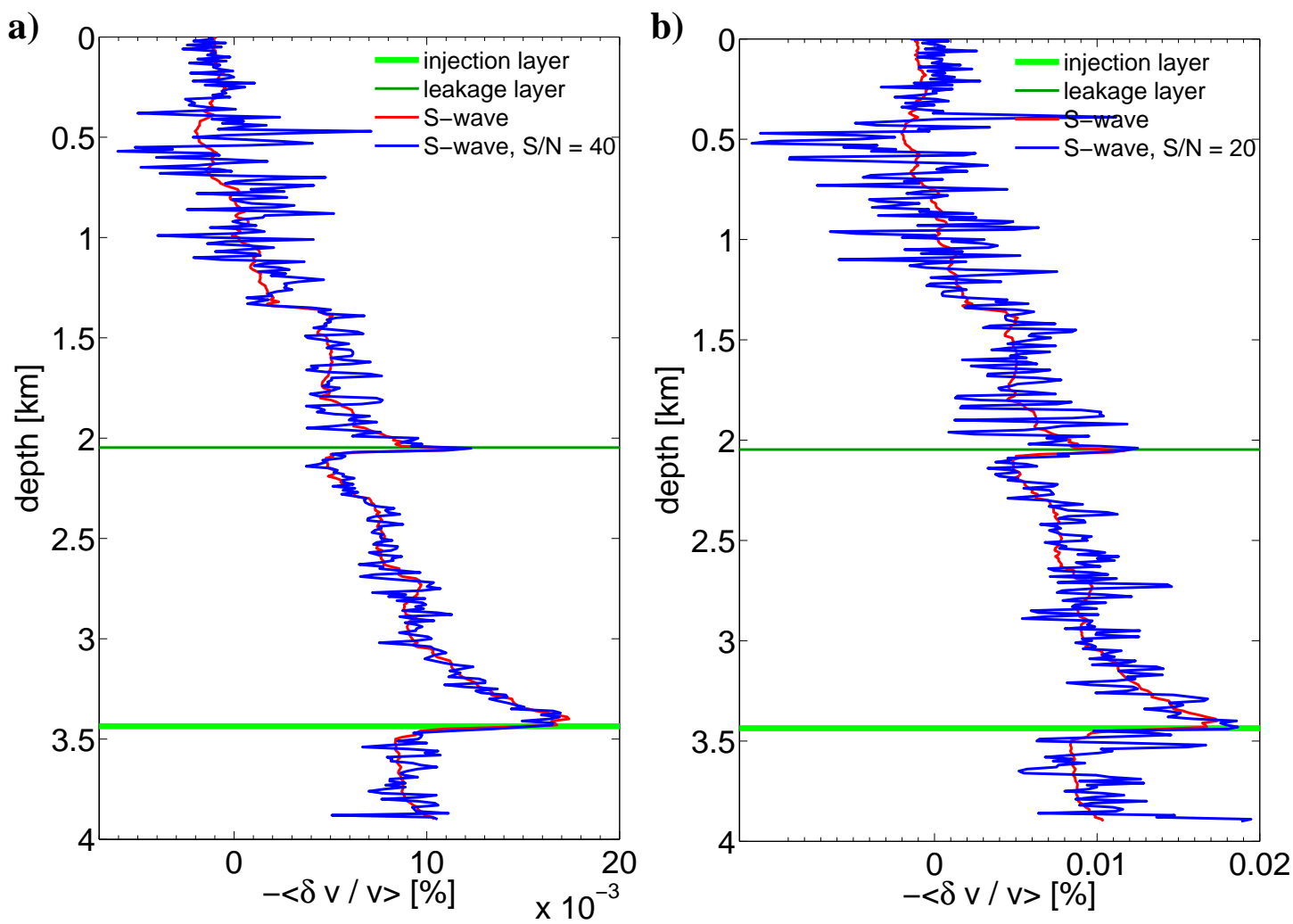

Figure 6.10: Mean velocity change of data contaminated with white noise of S/N-ratio of 40 (a) and 20 (b). The red curve in a) and b) show changes for the same data without noise. They are calculated on the leakage model (fig. 6.9 a). 
the deeper part from about $2000 \mathrm{~m}$ downwards. Here only minor, high frequent fluctuations superimpose the general trend. The peaks resulting from the leakage and the velocity changes in the reservoir and nearly all fluctuations in the noise-free data can be identified again. Above $2000 \mathrm{~m}$, which is the region above the first two strong reflection areas, amplitudes are fluctuating more strongly even though the $\mathrm{S} / \mathrm{N}$-ratio is better for the upper part of the model (fig. 3.9). However, this $\mathrm{S} / \mathrm{N}$-ratio is calculated at the first arrival times. For reflections traveling upwards from the reservoir and so appearing later in the coda, the $\mathrm{S} / \mathrm{N}$-ratio becomes much worse, because their amplitudes are very small compared to the first arrivals in this depth. When the upgoing wavelets pass the strong impedance contrast in $2050 \mathrm{~m}$ only a small amount of energy is transmitted and the $\mathrm{S} / \mathrm{N}$-ratio for this wavelet is very small above this interface.

For an S/N-ratio of 20 the fluctuations become much stronger and only the general trend of increasing mean velocity changes from the surface to the top reservoir is preserved. The fluctuations, which are again larger for the upper $2000 \mathrm{~m}$, completely swallow the mean velocity changes indicating the leakage area. However, the increase towards the reservoir depth is still displaying the upper limit of the $\mathrm{CO}_{2}$ cloud. Mean P-wave velocity changes are slightly more strongly affected. This test shows quite well that the threshold of $\pm 0.01 \%$ is justifiable.

\subsubsection{Shift}

Shifts in the receiver positions pose larger problems to coda wave interferometry. Figure 6.11 displays mean velocity changes for the leakage model (reservoir: $\Delta v_{p}=$ $-2 \%$ and $\Delta v_{s}=-2.25 \%$, leakage: $\Delta v_{p}=\Delta v_{s}=-4 \%$ ) with (blue and red) and without shifted receivers (light blue and pink). The receivers were shifted to greater depth by $1 \mathrm{~m}$.

After the first $300 \mathrm{~m}$ (which are not reliable, as they were damped during wavefield separation) the superordinate trend of the data from the shifted receivers is similar to the one of the unshifted data with an increase from low values at shallow depth to a peak value at the top of the reservoir, just shifted by about $-0.008 \%$ (P-wave) and $-0.0125 \%$ (S-wave). This makes it still possible to identify the upper limit of the velocity changed regions. But strong and abrupt changes also occur in other depth regions that could lead to misinterpretations (e.g. in $1350 \mathrm{~m}$ depth). This misinterpretation will be more likely, if not all but just part of the receivers are shifted. A distinct interpretation and a correct prediction of the appearance of $\mathrm{CO}_{2}$ from this result is difficult. 


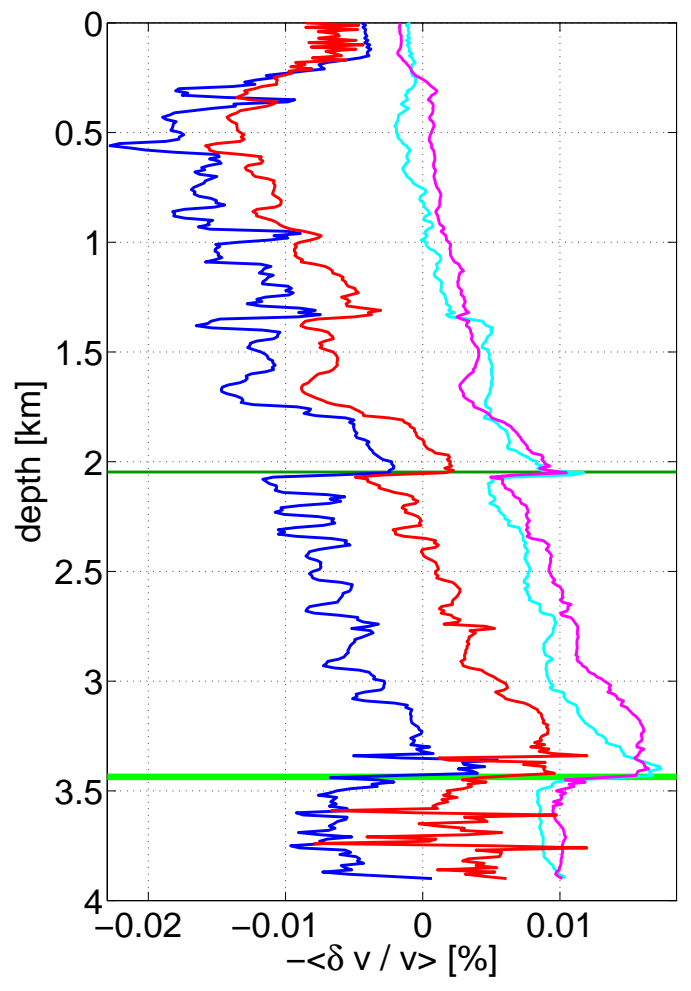

Figure 6.11: Mean velocity change for receivers shifted by $1 \mathrm{~m}$ (red and blue) and without shift (pink and light blue).

\subsection{Conclusion}

Coda wave interferometry applied to synthetic data calculated on a simplified Altmark model showed that a number of parameters influence the size of mean velocity changes and makes a quantitative interpretation of the results difficult. Beside a direct change of the velocity in the reservoir, the size of the changed zone and the source receiver offset is also influencing mean velocity changes. Large $\mathrm{CO}_{2}$ expansion zones increase the signal as lots of wavelets are shifted. When small zones occur, e.g. when looking for leakages, large velocity changes are necessary in order to produce measurable mean velocity changes. Large offsets give a sharper but only slightly stronger signal at top reservoir, while in the shown case even a decrease for $\mathrm{P}$-wave velocity changes occurs. However for other models it gave an increase in both components due to a focusing of the waves that travelled the changed region.

The method is also very sensitive to offsets of the $\mathrm{CO}_{2}$-cloud to receivers. When the $\mathrm{CO}_{2}$ has enclosed the observation well, the detection of velocity changes of about $1.5 \%$ to $2 \%$ are possible in the Altmark case, while offsets of just $100 \mathrm{~m}$ decrease the 
maximum mean velocity change significantly. A velocity changed zone surrounding the observation well is the optimum situation as the top of this zone is accurately localized by the maximum in the horizontal component.

When leakages occur, velocity changes are often quite large, as formation water exists most likely in the corresponding layers. The leakage tests showed that already small leakage zones can be registered when they occur in the vicinity of the observation well.

In the configuration used in our tests, S/N-ratios of about 40 are nearly not affecting the estimation of mean velocity changes. At S/N-ratios of about 20 the results become rapidly worse and a detection of the depth of the $\mathrm{CO}_{2}$-cloud becomes difficult. This shows that interferometry, especially when using large time windows $\left(2 t_{w}\right)$, is not very susceptible concerning noise above $\mathrm{S} / \mathrm{N}$-ratios of 20 . However, these tests were carried out on attenuation-free data and a quantitative interpretation of these noise tests is of limited validity. Damping will especially decrease the amplitude of the coda, as late arriving wavelets travel longer ways through the subsurface. This might strongly affect the determination of mean velocity changes especially when dealing with large offsets. This needs further examination.

Shifts in receiver position cause problems. Even if all receivers are shifted by the same value the interpretation of calculated mean velocity changes is difficult.

CWI in this configuration does not make use of the full power of this method. Due to the small extension of the velocity changed zone (especially the low thickness), quite large velocity changes have to occcur to cause measurable mean velocity changes. Also the necessary configuration of receivers at the top and most likely inside and below the velocity changed region makes a use of CWI for the monitoring of $\mathrm{CO}_{2}$ in the reservoir nearly impossible. However, it can be a good addition for the monitoring of leakages. 



\section{Chapter 7}

\section{Rock Physics}

In the previous chapters the size of changes in rock properties was tested that are necessary to monitor them with different methods. The following chapter gives an estimation about changes that can be expected when injecting $\mathrm{CO}_{2}$ into the reservoir and if the required dimension of changes is realistic for the injection regime as it is planned for the Altmark gasfield. Therefore first the gas properties are calculated and in a second step the saturated rock properties. The estimation concentrates on dry reservoir conditions (no formation water in the pores), which is assumed for the Altensalzwedel block by the reservoir engineers of GDF Suez E\&P Deutschland $\mathrm{GmbH}$.

\subsection{Gas properties}

To calculate the physical properties of the involved single gases, which are carbon dioxide $\left(\mathrm{CO}_{2}\right)$ as injection gas and nitrogen $\left(\mathrm{N}_{2}\right)$ and methane $\left(\mathrm{CH}_{4}\right)$ as the two main components of the reservoir gas, equations of state from Span and Wagner (1996), Span et al. (2000) and Setzmann and Wagner (1991) were used. From the different equations existing in literature we decided for those, as they provide the highest accuracy. In Appendix A a comparison of two equations is presented, which are most used in hydrocarbon industry.

The validity of the functions for all three gases cover a range from below $0^{\circ} \mathrm{C}$ to several hundred degree at pressures up to several hundred MPa.

In the region of investigation (pressures around 4.5 $\mathrm{MPa}$ and temperatures around $125^{\circ} \mathrm{C}$ ), the errors for nitrogen are $\leq 0.05 \%$ for density and $\leq 0.3 \%$ for velocity, for methane are $\leq 0.07 \%$ for density and $\leq 0.15 \%$ for velocity and for carbon dioxide they are $\leq 0.05 \%$ for density and for velocity the errors are $\leq 1 \%$ and only in the vicinity of the critical point they exceed $1 \%$.

After calculating the properties of the single gases, the density and the velocity 

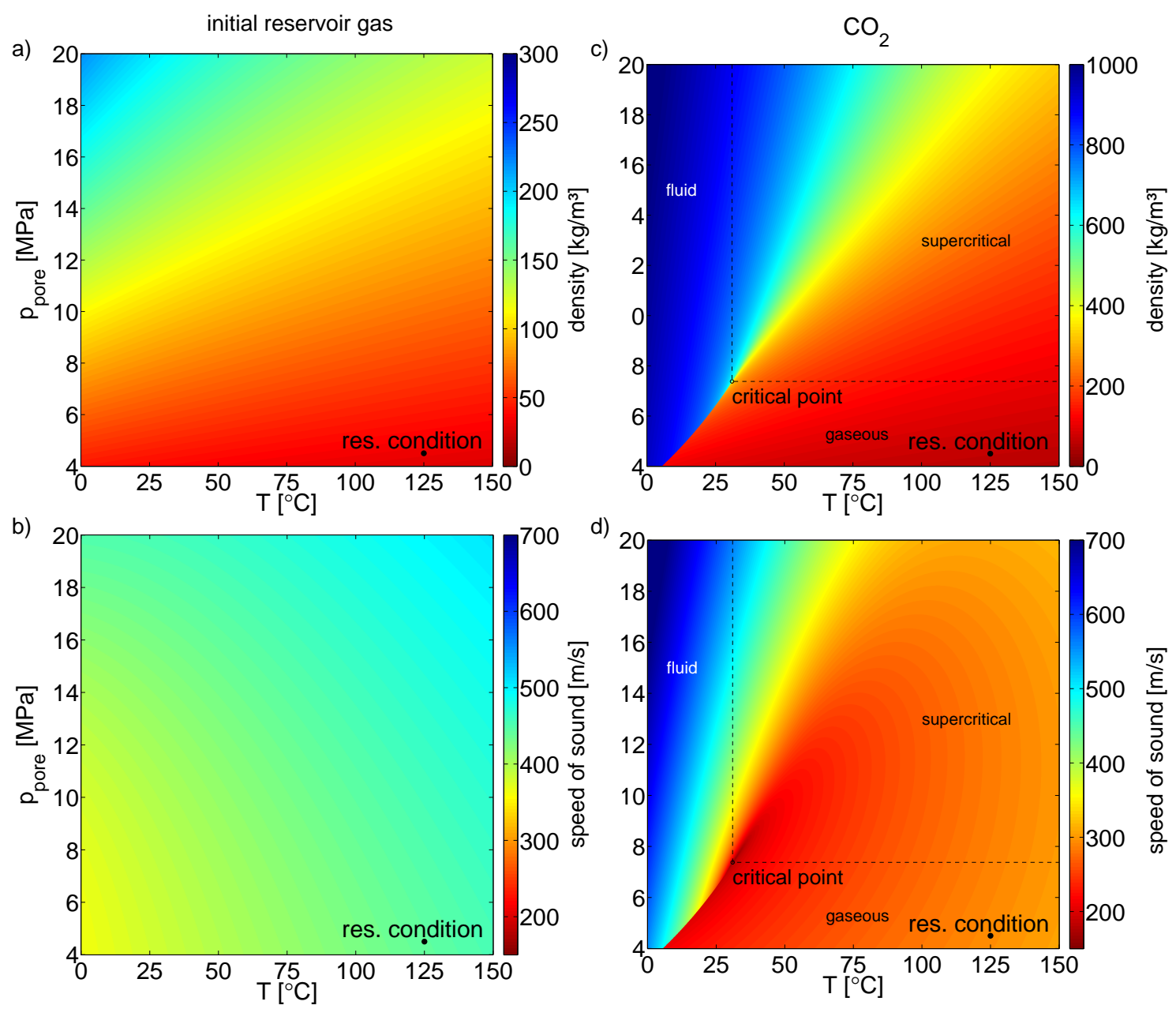

Figure 7.1: a) density and b) velocity of the initial gas mixture consisting of nitrogen and methane; c) density and d) velocity of pure $\mathrm{CO}_{2}$.

of the gas mixture can be determined, which is situated in the reservoir before $\mathrm{CO}_{2}$ injection. The density $\rho$ of a gas mixture is given by the molar average of the densities of the single gases

$$
\rho_{\text {mix }}=\sum_{i} x_{i} \rho_{i},
$$

and the velocity $v$ is determined by

$$
v_{m i x}=\sqrt{\frac{K_{m i x}}{\rho_{\text {mix }}}},
$$

with

$$
\frac{1}{K_{\text {mix }}}=\sum_{i} \frac{x_{i}}{K_{i}} .
$$


where $K$ is the bulk modulus and $x_{i}$ are the percentages of the gas components and add up to unity $\left(\sum_{i} x_{i}=1\right)$. From these equations we got the physical properties of the initial reservoir gas mixture and the injection gas as it is shown in the phase diagrams (fig. 7.1).

Figure $7.1 \mathrm{a}$ ) shows the density and figure $7.1 \mathrm{~b}$ ) the velocity of the reservoir gas as function of pressure and temperature. Both components of the reservoir gas are far away from their critical points, which are at $T_{c}=-83^{\circ} \mathrm{C}$ and $p_{c}=4.599 \mathrm{MPa}$ for methane and at $T_{c}=-147^{\circ} \mathrm{C}$ and $p_{c}=3.3958 \mathrm{MPa}$ for nitrogen, and the gas mixture in the reservoir should be in critical state (or near critical state for the methane component). This is why no strong changes in the properties occur in the shown pressure and temperature range. The critical point of $\mathrm{CO}_{2}$ lies within the demonstrated range $(7.1 \mathrm{c}$ and $\mathrm{d})$. In the vicinity of the critical point strong property changes occur for small temperature and/or pressure changes. And also for the region around the reservoir conditions, the property changes of $\mathrm{CO}_{2}$ due to pressure and/or temperature changes are larger than those of the reservoir gas and so the differences between the initial and the injected gas will increase with increasing pore pressure.

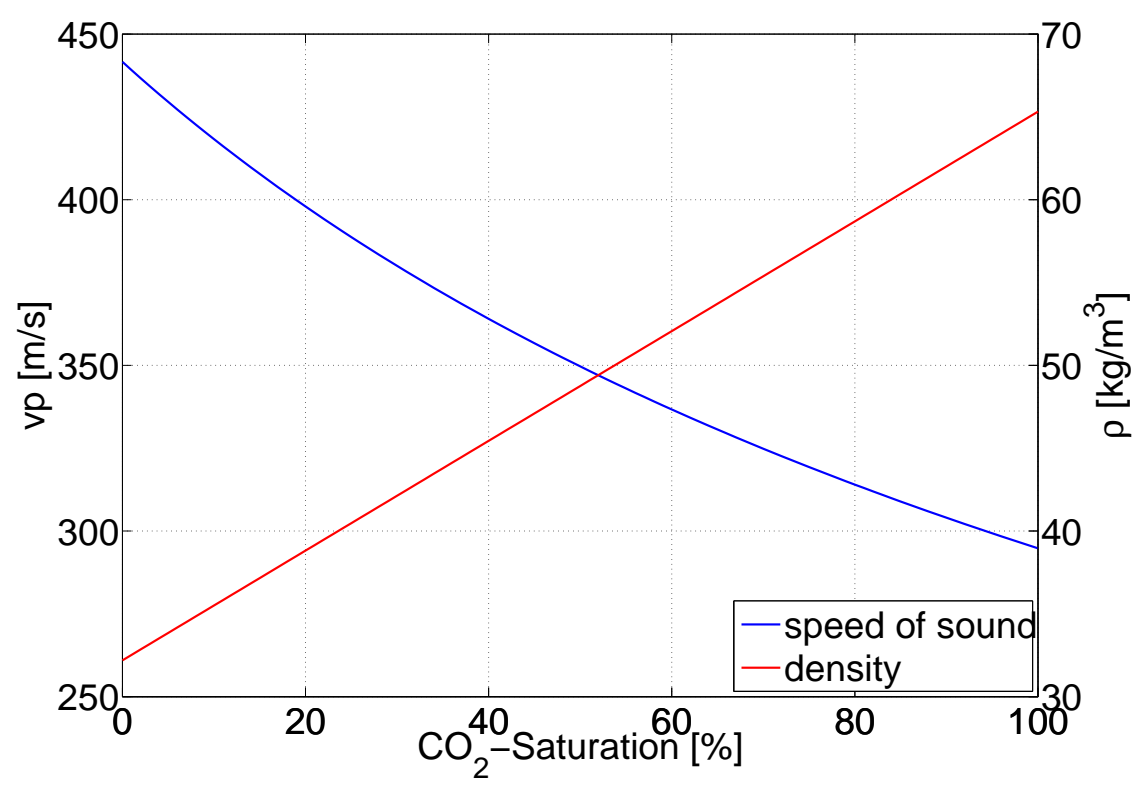

Figure 7.2: Speed of sound and density of the gas mixture as function of the $\mathrm{CO}_{2}$ saturation.

Nevertheless, the $\mathrm{CO}_{2}$ in this pilot test will be injected in gaseous state and also after injection stay like that, as the expected maximum increase in pore pressure is less than $2 M P a$. For larger tests or injection in an industrial dimension the pore pressure changes will easily become much larger. In any case the injection process is 
expected to be isothermal. This is due to the high contrast in specific heat capacities of the injection gas and the reservoir rock. But nevertheless it is also wanted, as cooling of rocks can induce fracs, that could untight the cap rock. So the injected $\mathrm{CO}_{2}$ will be heated up to approximately $8^{\circ} \mathrm{C}$ at the surface, further heats up on its way down the well and reaches bottom hole at reservoir temperature. Also the low pore pressures can cause a cooling of the injection gas due to the Joule-Thomson effect (until the point of freezing). So also the injection rate and the injection pressure have to be controlled very carefully.

During and after the injection process $\mathrm{CO}_{2}$ will displace the initial reservoir gas. But in the vicinity of the injection front and due to some smaller pores, where the reservoir gas is trapped and can not be easily pushed out, the gases will mix up. So it is more realistic to estimate the changes in rock properties not by replacing the reservoir gas by pure $\mathrm{CO}_{2}$ but by a mixture with high $\mathrm{CO}_{2}$ content. Figure 7.2 shows the speed of sound and density of the mixture of the reservoir gas and $\mathrm{CO}_{2}$ as a function of $\mathrm{CO}_{2}$-saturation at initial reservoir conditions (pore pressure of $4.5 \mathrm{MPa}$ and temperature of $125^{\circ} \mathrm{C}$ ). The density and the velocity of the mixture are again calculated by equations 7.1 and 7.2 .

As expected from the equations 7.1 - 7.3 the density changes linear, while the velocity shows a more complicated manner. Reservoir simulations made clear, that the largest part of the $\mathrm{CO}_{2}$ cloud will have a $\mathrm{CO}_{2}$ content of $80 \%$ or more. So in the following calculations a gas mixture of $80 \% \mathrm{CO}_{2}$ and $20 \%$ of the initial gas mixture will be used.

\section{2 $\quad$ Saturated rock properties}

To estimate the changes of the rock properties in the subsurface, the determination of the saturated rock properties is needed, i.e. the properties of a porous rock saturated with either of the gases. This procedure is explained in lots of textbooks as e.g. Mavko et al. (1998). The saturated density $\left(\rho_{\text {sat }}\right)$ is again given by the molar average of the density of the rock matrix $\left(\rho_{\text {matrix }}\right)$ and the density of the fluid in the pores $\left(\rho_{f l}\right)$

$$
\rho_{\text {sat }}=(1-\phi) \rho_{\text {matrix }}+\phi \rho_{f l},
$$

where $\phi$ indicates the porosity of the rock. To get the P-wave velocity, first the saturated bulk modulus $\left(K_{\text {sat }}\right)$ has to be calculated. This is done by the Gassmann equations

$$
K_{s a t}=\frac{K_{0}\left(\frac{K_{d r y}}{K_{0}-K_{d r y}}+\frac{K_{f l}}{\phi\left(K_{0}-K_{f l}\right)}\right)}{1+\frac{K_{d r y}}{K_{0}-K_{d r y}}+\frac{K_{f l}}{\phi\left(K_{0}-K_{f l}\right)}}
$$




$$
\mu_{\text {sat }}=\mu_{d r y} .
$$

Here $K_{d r y}$ is the dry bulk modulus and $\mu_{d r y}$ the dry shear modulus, which describe the particular modulus of the dry rock, without filling of the pores. $K_{0}$ is the grain modulus, the bulk modulus of the crystalline matrix. $K_{f l}$ is the bulk modulus of the pore fluid, which is the nitrogen-methane gas mixture in the first and carbon dioxide in the second case. The saturated P-wave velocity is then determined by

$$
v_{p, s a t}=\sqrt{\frac{K_{s a t}+\frac{4}{3} \mu_{s a t}}{\rho_{s a t}}}
$$

while the shear wave velocity is only affected by the change in density

$$
v_{s, s a t}=\sqrt{\frac{\mu_{s a t}}{\rho_{s a t}}} .
$$

Petrographic x-ray and thin-section analysis, provided by GDF Suez E\&P Deutschland $\mathrm{GmbH}$, yield the mineral content of the reservoir layers. From these measurements we took the data of the samples in a depth of $3408.0 \mathrm{~m}$ to $3503.9 \mathrm{~m}$, the depth range of the reservoir layers. All samples in this range were taken into account to calculate the average volume fraction of the particular minerals. For the following calculations only the minerals with an average content of more than $1 \%$ volume fraction were used and scaled, so that their sum gives 100\%. With the volume fraction $\mathrm{f}(\mathrm{i})$ of the constituents and their bulk $(\mathrm{K}(\mathrm{i}))$ and shear moduli $(\mu(i))$ (Carmichael, 1990) the grain bulk modulus $K_{0}$ was calculated as the average of the upper $\left(K_{u p}\right)$ and lower $\left(K_{\text {low }}\right)$ Hashin-Shtrikman bounds as it is explained e.g. in Mavko et al. (1998)

$$
K_{0}=\frac{K_{u p}+K_{\text {low }}}{2}
$$

with

$$
\begin{aligned}
K_{u p} & =\frac{1}{\sum_{i}\left(\frac{f(i)}{K(i)+\frac{4}{3} \mu_{\max }}\right)}-\frac{4}{3} \mu_{\max } \\
K_{\text {low }} & =\frac{1}{\sum_{i}\left(\frac{f(i)}{K(i)+\frac{4}{3} \mu_{\min }}\right)}-\frac{4}{3} \mu_{\text {min }} .
\end{aligned}
$$

In the equation of the Hashin-Strikman bounds, $\mu_{\max }$ and $\mu_{\min }$ indicate the highest and lowest shear modulus of all constituents appearing in the rock sample.

The dry moduli and density were taken from two rock samples from the reservoir of the Peckensen block that is also part of the Altmark gas field and located north western of the Altensalzwedel block. This block has the same stratigraphy as the Altensalzwedel block, so that a transfer of the data is legitimately. 
The properties of the samples were obtained from laboratory measurements, which were conducted and provided by Dr. J. Kummerow (personnel communication). Both samples are of sandstone with high porosities of $10.336 \%$ for sample Pes4 1612 and of $22.085 \%$ for sample Pes4 $1622 \mathrm{~b}$ and have a matrix density $\left(\rho_{\text {matrix }}\right)$ of $2674 \frac{\mathrm{kg}}{\mathrm{m}^{3}}$ and $2647 \frac{\mathrm{kg}}{\mathrm{m}^{3}}$, respectively. The pore pressure dependence of the velocity of dry rocks is just approximated, as the measuring of this effect in the laboratory failed. It is described by the Eberhard-Phillips equation ( Zimmermann et al. (1986); Eberhard-Phillips et al. (1989); Shapiro (2003))

$$
v_{p, s}\left(P_{d}\right)=A_{p, s}+K_{p, s} P_{d}-B_{p, s} \exp ^{-D_{p, s} P_{d}}
$$

where $v_{p, s}$ is the $\mathrm{P}$-/S-wave velocity and $A_{p, s}, K_{p, s}, B_{p, s}$ and $D_{p, s}$ are coefficients for either $\mathrm{P}$ - or S-waves. $P_{d}$ is the differential pressure, the difference of the confining pressure and the pore pressure $P_{d}=P_{c}-P_{\text {pore }}$. The coefficients $K_{p, s}, B_{p, s}$ and $D_{p, s}$ were approximated by taking the arithmetic mean values measured for other sandstones published by Biryaltseva (2010). The slope is shifted to the measured velocities of the Peckensen samples by varying $A_{p, s}$. The coefficients are listed in table 7.1. This approximation enables us to calculate the dry bulk $\left(K_{d r y}\right)$ and shear modulus $\left(\mu_{d r y}\right)$ of the two samples for given differential pressures.

\begin{tabular}{|c|c|c|}
\hline & Pes4 1612 & Pes4 1622b \\
\hline \hline$A_{p}$ & 4.055 & 2.92 \\
\hline$K_{p}$ & 0.00115 & 0.00115 \\
\hline$B_{p}$ & 1.3 & 1.3 \\
\hline$D_{p}$ & 0.1 & 0.1 \\
\hline$A_{s}$ & 2.475 & 1.63 \\
\hline$K_{s}$ & 0.0007 & 0.0007 \\
\hline$B_{s}$ & 0.8 & 0.8 \\
\hline$D_{s}$ & 0.1015 & 0.1015 \\
\hline
\end{tabular}

Table 7.1: Parameter to calculate pore pressure dependence of velocity.

\begin{tabular}{|c|c|c|}
\hline & Pes4 1612 & Pes4 1622b \\
\hline \hline$\phi$ & $10.336 \%$ & $22.085 \%$ \\
\hline$\rho$ & $2677.327 \frac{\mathrm{kg}}{\mathrm{m}^{3}}$ & $2653.110 \frac{\mathrm{kg}}{\mathrm{m}^{3}}$ \\
\hline$v_{p}$ & $4360.427 \frac{\mathrm{m}}{\mathrm{s}}$ & $3127.793 \frac{\mathrm{m}}{\mathrm{s}}$ \\
\hline$v_{s}$ & $2509.427 \frac{\mathrm{m}}{\mathrm{s}}$ & $1663.755 \frac{\mathrm{m}}{\mathrm{s}}$ \\
\hline
\end{tabular}

Table 7.2: Physical properties of samples at initial reservoir conditions $\left(p_{\text {pore }}=\right.$ 4.5MPa, $T=125^{\circ} \mathrm{C}$, pore fluid: gas mixture).

Based on these information, the properties of the saturated rock are calculated first under initial reservoir conditions, which are a nitrogen-methane gas mixture as pore fluid, a pore pressure of $4.5 M P a$, a confining pressure of $60 M P a$ and a temperature of $125^{\circ} \mathrm{C}$ (GDF Suez E\&P Deutschland GmbH, personnel communication). Table 7.2 shows the results. Afterwards velocities and density are calculated for the rock saturated with $80 \% \mathrm{CO}_{2}$ and $20 \%$ of the initial reservoir gas and for different pore pressures as the pore pressure is expected to change during injection. The change in density and velocities in the subsurface due to $\mathrm{CO}_{2}$ injection is then 
determined by normalizing these values to the initial reservoir conditions (values in table 7.2). Figure $7.3 \mathrm{a}$ ) and b) shows these changes as function of pore pressure for rock properties of sample Pes4 1612 and Pes4 1622b, respectively.
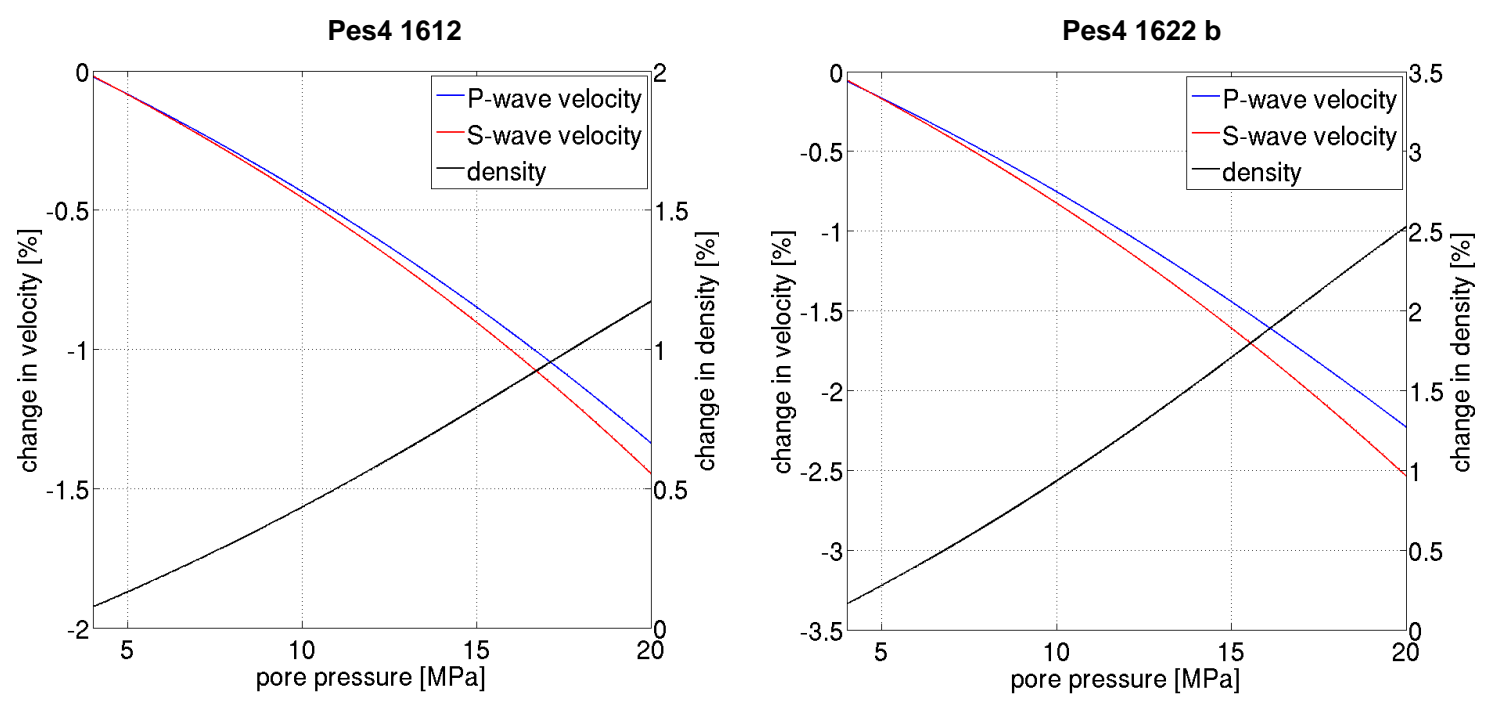

Figure 7.3: Change in P- and S-wave velocity and density for rock properties of a) sample Pes4 1612 and b) Pes4 1622b as function of pore pressure. The properties are calculated for a pore fluid of $80 \% \mathrm{CO}_{2}$ and $20 \%$ of the initial reservoir gas and are normalized to the initial reservoir conditions.

The changes of the properties of both samples show a similar behaviour, but with approximately a factor 2 larger for the sample of higher porosity (Pes4 1622b). When injecting $\mathrm{CO}_{2}$ the velocities decrease and the density increases, as the velocity of $\mathrm{CO}_{2}$ is lower and its density higher than that of the nitrogen-methane reservoir gas (fig. 7.1). This trend further proceeds if the pore pressure increases.

If only replacing the pore fluid and keeping the pressure constant at $4.5 \mathrm{MPa}$, sample Pes 1612 shows only very little velocity changes of less than $-0.1 \%$ (fig. 7.3 a). With additional pressure changes of up to $20 \mathrm{MPa}$ velocities decrease to $-1.45 \%$ for S-wave velocity and $-1.3 \%$ for P-wave velocity. The density also changes only slightly from about $+0.1 \%$ without pressure changes to $+1.2 \%$ for a pore pressure of $20 M P a$.

For rock properties of sample Pes4 1622b the changes are higher as its porosity is higher and its dry bulk modulus is lower. Here the replacement of the pore fluid changes the velocity by not more than $-0.1 \%$ and the density by $+0.2 \%$ (fig. $7.3 \mathrm{~b}$ ). With increasing pore pressure the density change increases to $+2.5 \%$ for $20 \mathrm{MPa}$. The S-Wave velocity decreases to $-2.5 \%$ and the $\mathrm{P}$-wave velocity to $-2.2 \%$ for pore pressures of $20 M P a$.

To get information about the change in reflection wave amplitude we have to 


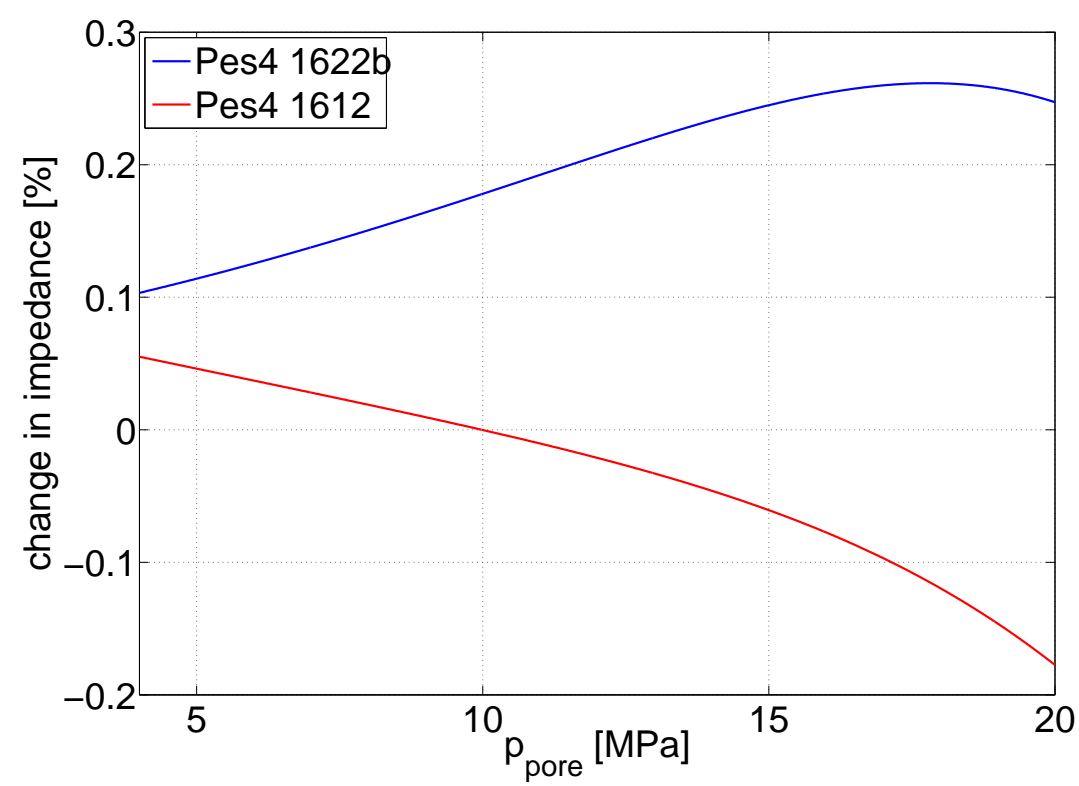

Figure 7.4: Change in impedance of the two rock samples saturated with a mixture of $80 \% \mathrm{CO}_{2}$ and $20 \%$ at different pressures. The impedances are standardized to the impedance of the rock samples under prior reservoir conditions.

investigate the acoustic impedance, i.e. the product of velocity and density, which is shown in Figure 7.4 as function of pore pressure. It is again standardized to the prior reservoir conditions. For Pes4 1612 the impedance changes by $+0.05 \%$ due to the replacement of the pore fluid. For additional pressure changes the impedance change decreases, is zero at a pore pressure of $10 \mathrm{MPa}$ and further decreases to $-0.18 \%$ for $20 \mathrm{MPa}$. The impedance of sample Pes4 1622b shows a different behaviour. It changes by about $+0.1 \%$ due to $\mathrm{CO}_{2}$ injection and afterwards further increases for increasing pore pressures until a maximum is reached at about $18 M P a$ with a change of $+0.26 \%$. Then it slightly decreases again.

\subsection{Conclusion}

These results indicate that in the Altmark reservoir only small changes in rock properties of a few per mill occur from the replacement of reservoir gas by $\mathrm{CO}_{2}$. To generate larger changes in the dimension of $1 \%$ or more additional large changes in the pore pressure and/or the reservoir temperature are necessary. Larger changes can be expected in reservoirs with lower temperatures, where conditions are closer to the critical point of $\mathrm{CO}_{2}$. 


\section{Chapter 8}

\section{Discussion}

The initial goal of the active seismic within the CLEAN project was the design, execution and interpretation of seismic measurements attending the $\mathrm{CO}_{2}$ EGR pilot project CLEAN. Because the execution was stopped due to political complications, prestudies were intensified concerning changes of physical properties in the reservoir rocks due to $\mathrm{CO}_{2}$ injection, the generated changes in the seismic wavefield and their localization possibilities. These tests provided crucial information that found way into the experiment design. Further results from the "Reservoir Simulation" subproject were incorporated. Figure 8.1 shows a suggestion of an optimal experiment design for the monitoring of the reservoir and the overlying layers and aquifers as it would have been accomplished in the framework of this project if not stopped. However, this suggestion underlies the restrictions that hold for this project, which are the useable depth range in the observation well and the financial frame of the project.

The observation well is limited by a cement bonding plug that seals the perforated bottom of the well in order to avoid an escape of $\mathrm{CO}_{2}$ after breakthrough. The upper part of the well has an inner, uncemented tubing. Thus for VSP-experiments only the range between about $2200 \mathrm{~m}$ and $3300 \mathrm{~m}$ is useable. A receiver-chain of $163 \mathrm{C}$ geophones with intertool spacing of $10 \mathrm{~m}$ was available. The tool covers a distance of $150 \mathrm{~m}$. In order to cover the complete measuring range in the well it has to be lifted to 7 depth positions.

The financial frame of the project allows working with Mertz Hemi vibrators from DMT for a maximum of 19 days in the field. But as very little financial reserves exist, the plan allows only source points on official streets and tracks and a safety zone of $50 \mathrm{~m}$ from buildings in order to avoid damage.

In figure 8.1 the reservoir is marked by the orange line, the injection wells are S13 and S17 (red), production wells are S16 and S14 (blue) and the observation well is S1 (green). The crosses denote the shot positions. They are oriented star 


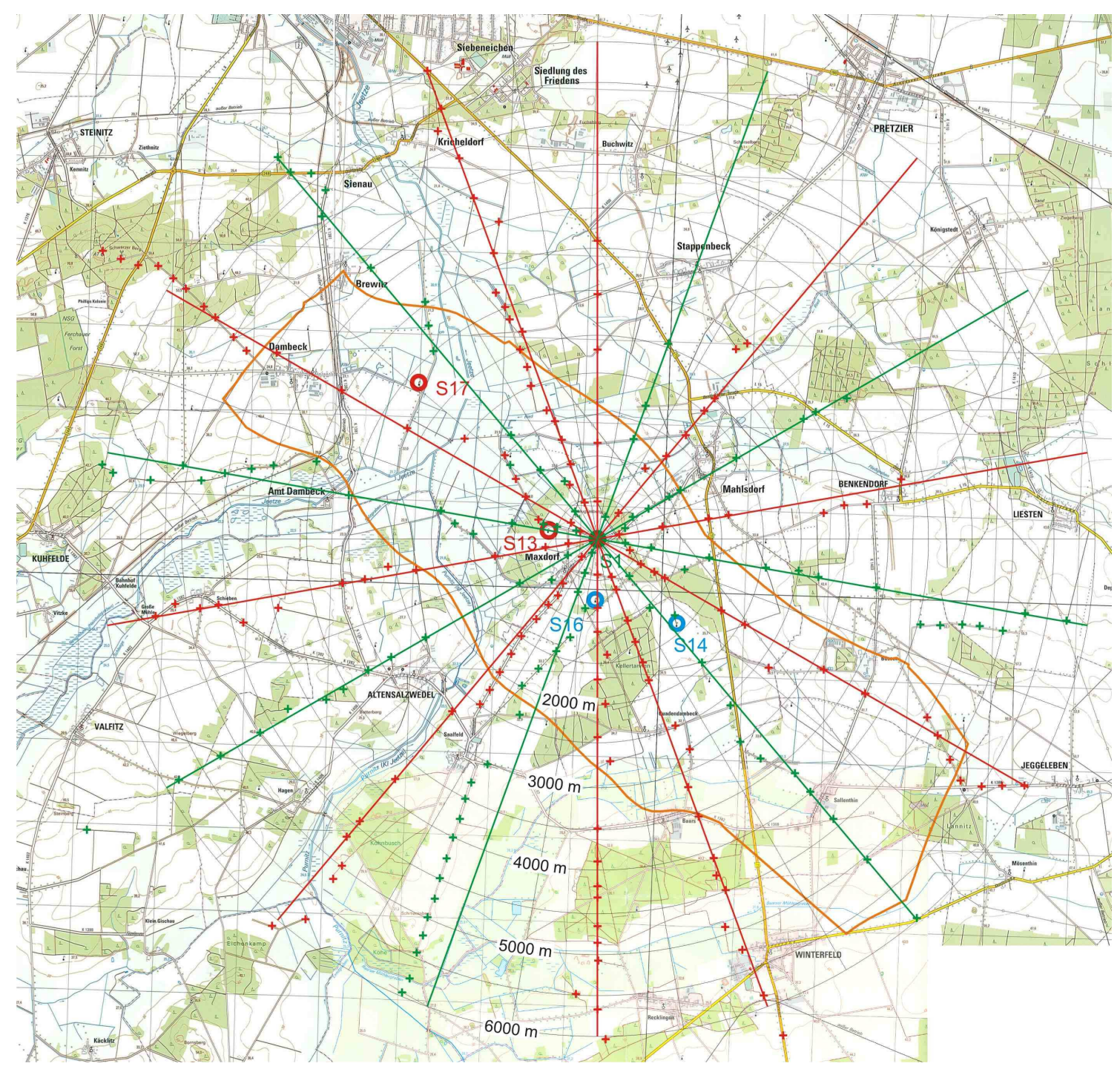

Figure 8.1: Suggestion for the seismic monitoring attending the $\mathrm{CO}_{2}$-injection in the Altmark. 
like, crossing the observation well. The single lines have a spacing of $20^{\circ}$. Different colours are only meant to distinguish between neighbouring profiles. We tried to adhere a shot point spacing of $250 \mathrm{~m}$ that was proved to be sufficient in migration tests, but not always possible without leaving streets and tracks. The maximum offsets are up to $6000 \mathrm{~m}$, in north and north-eastern directions smaller, just up to $2400 \mathrm{~m}$. This is due to the smaller extension of the reservoir in that direction and the results from reservoir simulations (Awemo, pers. com.). They predict that the propagation of the injected $\mathrm{CO}_{2}$ tends southeast towards the production wells S16 and S14 following the reservoir pressure gradient with a maximum propagation of about $1000 \mathrm{~m}$ after 2 years (fig. 8.2). The reflection points will approximately cover the first $500 \mathrm{~m}$ of the reservoir around the observation well in north and northeastern directions and about $1500 \mathrm{~m}$ in other directions of large offsets. Withit it covers the expected expansion area of the $\mathrm{CO}_{2}$ during the first 2 years. This survey design can also be used to monitor the aquifers above the reservoir. In this case transmitted waves (down-going wavefield) have to be analysed for the monitoring of aquifers above $2200 \mathrm{~m}$.

The survey has 247 shot points. The vibrators have to emit at all these points at each of the 7 depth positions of the receiver line. This gives a total number of

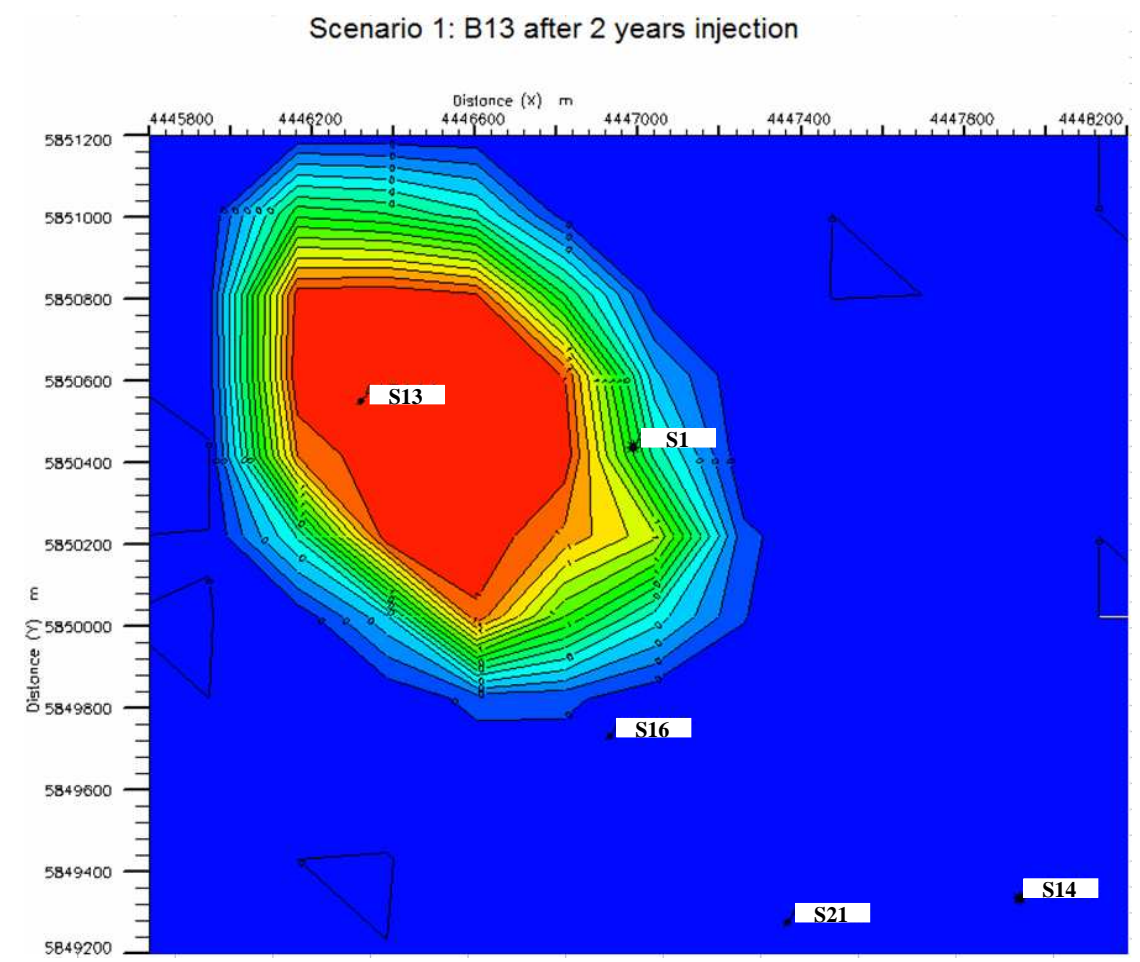

Figure 8.2: Map view of $\mathrm{CO}_{2}$ saturation distribution in one of the most permeable reservoir layers 2 years after injection started. 
1729 shots (247 shot points $\times 7$ depth positions of the VSP-tool). Due to the partly large distances between shot points, a number of about 100-120 shots per day is realistic, which gives a total of about 1900-2280 shot positions. The surplus can be economized or be used to compact the survey with some additional profile lines (thin black lines, $10^{\circ}$ spacing) or by closing some gaps on the profile lines, resulting from the absence of tracks, with shot positions on fields in order to receive a more regular fold distribution as it was shown to be necessary in the $\mathrm{CO}_{2}$-sequestration project in Ketzin (see chap. 2) and to use it as reserve for field damage.

With this survey geometry the detection and localization of impedance changes of about $0.25 \%$ or more are possible (e.g. $\Delta v_{p}=-1.5 \%, \Delta v_{s}=-1.7 \%$ and $\Delta \rho=$ $+1.8 \%$ ) as was shown by the analysis of amplitude changes. But rock-physical estimates show that pressure changes of up to $10 \mathrm{MPa}$ or additional temperature changes have to occur with the replacement of the pore gas in the reservoir rocks by $\mathrm{CO}_{2}$ in order to cause impedance changes of that dimension. However this is not realistic for injection of $100.000 t$ of $\mathrm{CO}_{2}$ as shown by reservoir simulations accomplished by Awemo et al. (pers. com.) as part of the CLEAN subproject "Reservoir Simulation". Their results are shown in figure 8.3 a) and b) in a cross section. They are adapted to the FD grid used in this study by horizontally flatening the layers, which is tolerable, as the vertical variations of the reservoir layers do not exceed $16 \mathrm{~m}$ on a distance of $2000 \mathrm{~m}$. Here maximum pressure changes are up to $+1 \mathrm{MPa}$ and occur only in the vicinity of the injection well $\mathrm{S} 13$. The $\mathrm{CO}_{2}$ saturation is quite high around the injection well $\mathrm{S} 13\left(90 \%\right.$ - 100\%) and the $\mathrm{CO}_{2}$ propagates beyond the observation well (fig. $8.3 \mathrm{~b}$ ). But the corresponding velocity changes that were calculated on base of pressure, saturation and porosity information, are approximately $1 / 10$ of the required (figure $8.3 \mathrm{c}$ ). Under these conditions a seismic monitoring of the $\mathrm{CO}_{2}$ propagation in the reservoir will fail. Higher impedance changes occur when $\mathrm{CO}_{2}$ replaces a fluid instead of a gas. This could be the case if formation water penetrated into the reservoir layers during production. In this case velocity changes of up to $-10 \%$ are realistic (Xue and Ohsumi, 2004).

But even if the reservoir is dry high prospects of success will be given to an indirect monitoring of the $\mathrm{CO}_{2}$. In this case the monitoring is focused on finding possible leakages, when $\mathrm{CO}_{2}$ escapes from the reservoir into overlying water-bearing layers and is going in solution and partly displaces the formation water. If no leakage is found the $\mathrm{CO}_{2}$ can be expected to be sealed in the reservoir. Therefore the aquifers above the reservoir and here especially the regions with higher leakage risk like folds and old wells have to be monitored.

But for detecting $\mathrm{CO}_{2}$ leakages the VSP-geometry is not optimal, as a large area has to be covered with seismic reflection points. Here a 3D-surface seismic array is 
a) Pressure difference (after 2 years minus initial) [MPa]

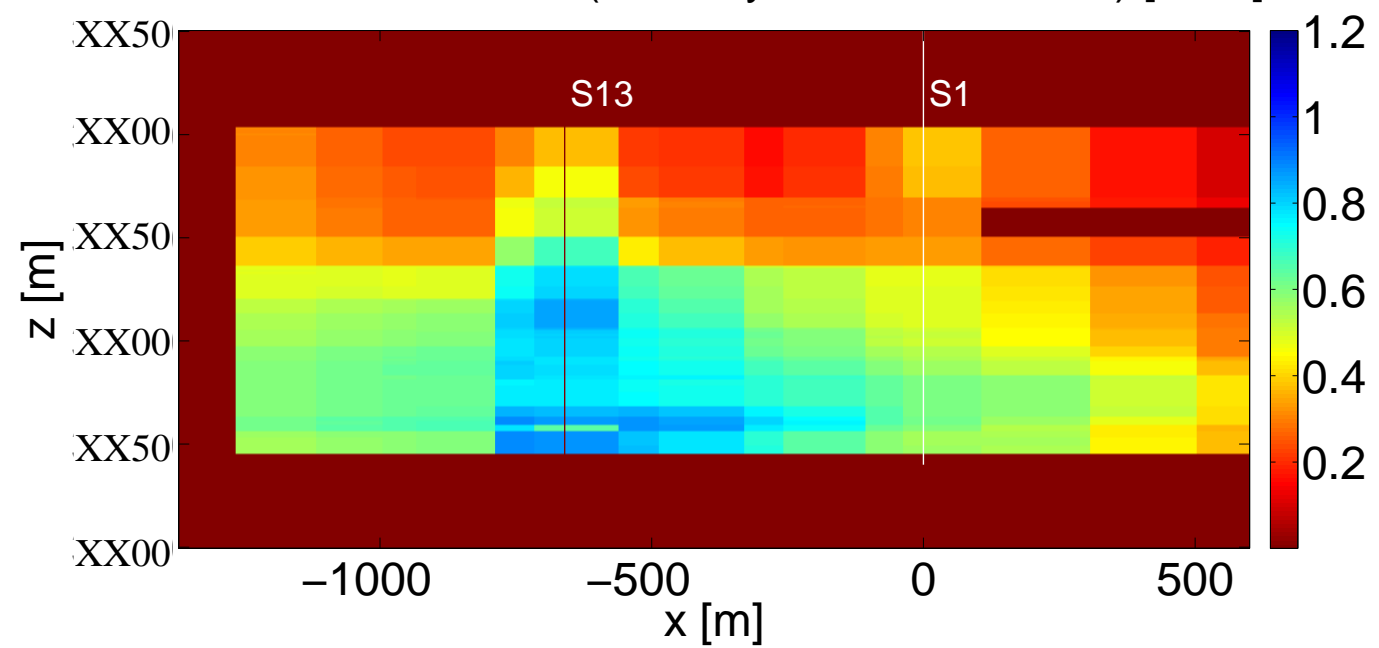

b) $\quad \mathrm{CO}_{2}$ saturation (after 2 years of injection) [\%]

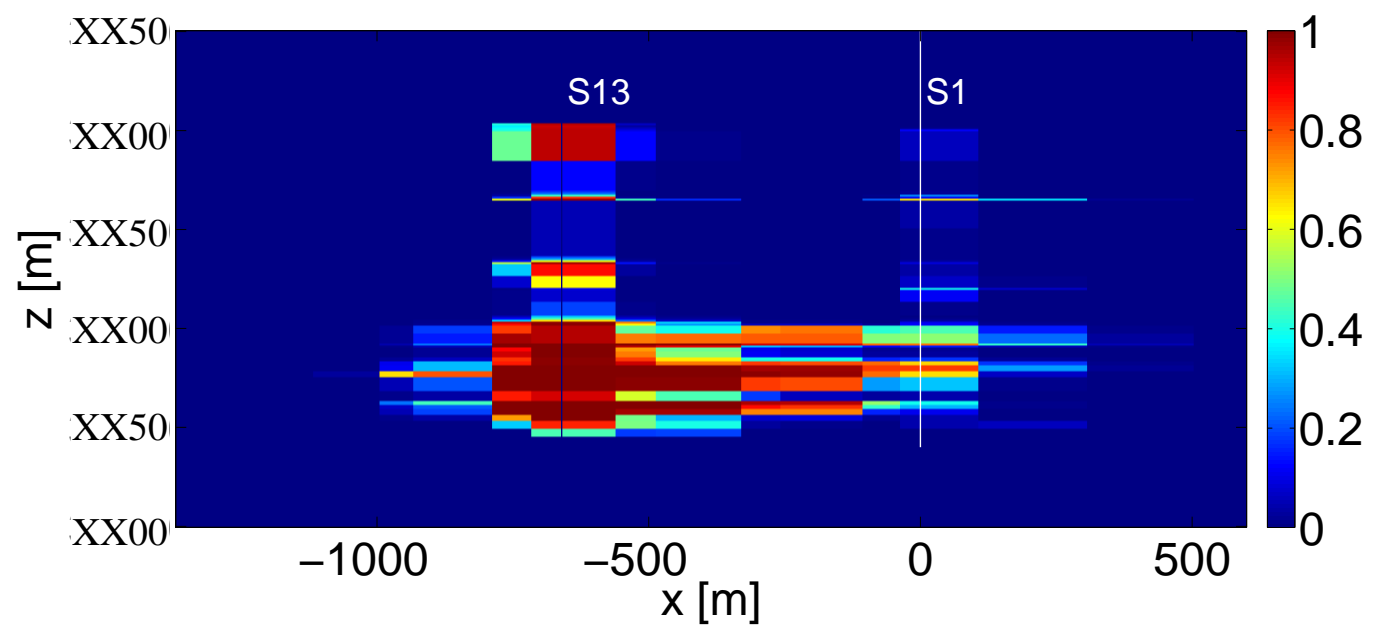

c) Change in P-wave velocity (after 2 years of injection) [\%]

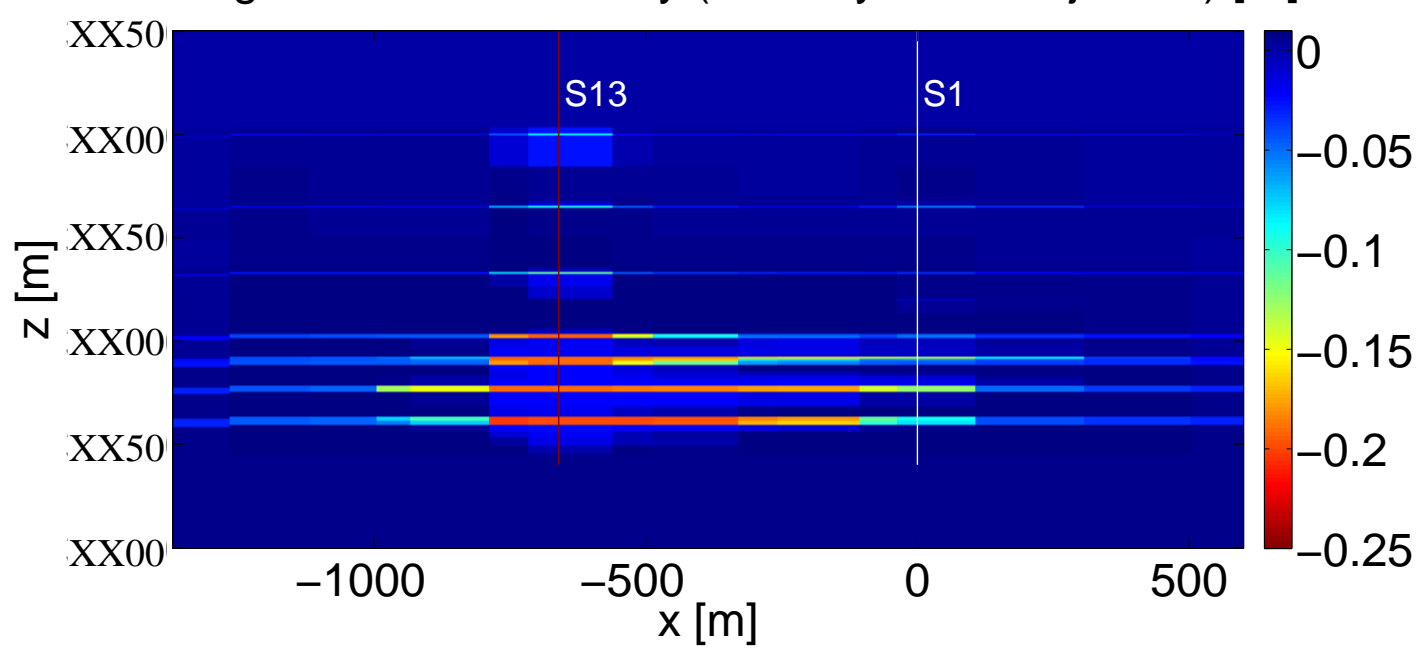

Figure 8.3: Pressure (a) and saturation simulation (b) for the Altensalzwedel reservoir and corresponding $\mathrm{P}$-wave velocity changes $(\mathrm{c})$. 
advisable.

Beside the effects of amplitude changes time shifts caused by velocity changes in the subsurface were analyzed on their interpretability. Therefore the method of coda wave interferometry was used. The tests showed that velocity changes of about $\pm 2 \%$ are necessary in our case to be detectable. They also showed, that the offset of the $\mathrm{CO}_{2}$ cloud to the observation well is crucial i.e. breakthrough conditions greatly improves the detectability. This procedure gives only a more or less one dimensional interpretation of velocity changes in an area around the observation well. Information about the three dimensional spatial distribution of the $\mathrm{CO}_{2}$ can only be given, when measuring on several wells. However, these information can be received easier, cheaper and with higher accuracy by a tracer gas monitoring. But the CWI method showed potential for the monitoring of leakages near existing wells in the reservoir. Old wells have a heightened leakage risk, as old cementation can contain fracs and possible pathways for the injected $\mathrm{CO}_{2}$. The $\mathrm{CO}_{2}$ can migrate upwards along those fracs and escape from the reservoir. On its way to the surface it passes a multitude of different rock formations from which some might be less permeable (e.g. clay layers) and will accumulate the $\mathrm{CO}_{2}$. Thereby the $\mathrm{CO}_{2}$ will go into solution and partly push away formation water, which can produce velocity changes of 4\%-10\%. These changes will most likely occur in the vicinity of the well and can be monitored with high accuracy by coda wave interferometry.

However coda wave interferometry, which is capable of detecting very small velocity changes if the velocity changed region is sufficient large, cannot be used to its full potential in this application due to the limited extension of the effected reservoir. In order to detect velocity changes in this case, the changes itself have to be considerably large.

Further studies could aim at a migration of the "difference sections", as are shown in figure 6.5, where higher velocity changes appear such that measurable time shifts also occur in the first reflections. 


\section{Bibliography}

Aki, K. and Richards, P. G. (1980). Quantitative Seismology: Theory and Methods. W. H. Freeman, San Francisco.

Anderson, S. and Newell, R. (2003). Prospects for Carbon Capture and Storage Technologies. Discussion paper 02-68, Recources for the Future, Washington, USA.

Arts, R. and Vandeveijer, V. (2011). The challenges of monitoring $\mathrm{CO}_{2}$ storage. The Leading Edge, 30, 1026-1033.

Batzle, M. and Wang, Z. (1992). Seismic properties of pore fluids. Geophysics, 57, 1396-1408.

Biryaltseva, T. (2010). Stress-dependent porosity and elastic properties of sandstones: Interpretation of laboratory meassurements. Ph.D. thesis, Freie Universität Berlin. master thesis.

Blanch, J. O., Robertsson, J. O. A., and Symes, W. W. (1993). Viscoelastic finitedifference modeling. Tech. rep. 93-04, Department of Computational and Applied Mathematics, Rice University, Houston, Texas.

Blanch, J. O., Robertsson, J. O. A., and Symes, W. W. (1995). Modelling of a constant Q: Methodology and algorithm for an effective and optimally inexpensive viscoelastic technique. Geophysics, 60 (1), 176-184.

Bode, S. (2003). Abatement costs vs. Compliance costs in Multi-Period Emission Trading - The Firms' Perspective. Hwwa discussion paper 230, Hamburgisches Welt-Wirtschafts-Archiv (HWWA), Hamburg Institute of International Economics.

Bohlen, T. (2002). Parallel 3-D viscoelastic finite difference seismic modelling. Computers $\& 6$ Geosciences, 28, 887-899. 
Bohlen, T. (2008). Parallel 2-D elastic/viscoelastic finite difference seismic modelling.

Buske, S., Gutjahr, S., and Sick, C. (2009). Fresnel volume migration of singlecomponent seismic data. Geophysics, 74 (6), WCA47-WCA55.

Campbell, A., Fryer, A., and Wakeman, S. (2005). Vertical seismic profiles - more than just a corridor stack. The Leading Edge, 24 (7), 694-697.

Carcione, J. M., Kosloff, D., and Kosloff, R. (1988). Wave propagation simulation in a linear viscoelastic medium. Geophys. J. Roy. Astr. Soc., 93, 393-407.

Carmichael, R. S. (1990). Practical Handbook of Physical Properties of Rocks and Minerals. CRC Press, Boca Raton, Fla., 2 edition.

Ćerveny̌, V. and Soares, J. E. P. (1992). Fresnel volume ray tracing. Geophysics, 57 (7), 902-915.

Chadwick, R. A., Arts, R., and Eiken, O. (2005). 4D seismic quantification of a growing $\mathrm{CO}_{2}$ plume at Sleipner, North Sea. Petroleum Geology: North West Europe and Global Perspectives - Proceedings of the 6th Petroleum Geology Conference, Geological Society, pages 1385-1399.

Chadwick, R. A., Williams, G., Delepine, N., Clochard, V., Labat, K., Sturton, S., Buddensiek, M.-L., Dillen, M., Nickel, M., Lima, A. L., Arts, R., Neele, F., and Rossi, G. (2010). Quantitative analysis of time-lapse seismic monitoring data at the Sleipner $\mathrm{CO}_{2}$ storage operation. The Leading Edge, 29, 170-177.

Eberhard-Phillips, D., Han, D.-H., and Zoback, M. D. (1989). Empirical relationships among seismic velocity, affective pressure, porosity and clay content in sandstone. Geophysics, 54, 82-89.

Emmerich, H. and Korn, M. (1987). Incorporation of attenuation into time-domain computations of seismic wave fields. Geophysics, 52 (9), 1252-1264.

Gerling, J. P. (2008). Geologische $\mathrm{CO}_{2}$-Speicherung als Beitrag zur nachhaltigen Energieversorgung. Bergbau, 10, 472-475. RDB e.V.

Hardage, B. A. (2000). Vertical Seismic Profiling: Principles, volume 14 of Handbook of Geophysical Exploration. Elsevier Science Ltd., Oxford, GB, UK, 3rd edition.

Ivandic, M., Yang, C., Lüth, S., Cosma, C., and Juhlin, C. (2012). Time-lapse analysis of sparse 3D seismic data from the CO2 storage pilot site at Ketzin, Germany. Journal of Applied Geophysics, 84, 14-28. doi: 10.1016/j.jappgeo.2012.05.010. 
Johnston, P. and Santillo, D. (2002). Carbon Capture and Sequestration: Potential Environmental Impacts. Proceedings of IPCC Workshop on Carbon Dioxide Capture and Storage, 18-21 November 2002.

King, A. D. (2004). Climate change science: adapt, mitigate, or ingnore. Science, 303, 176-177.

Kuzminski, R., Charters, B., and Galbraith, M. (2009). Processing Considerations for 3D VSP. CSEG Recorder, 34 (4), 30-40.

Kühn, M., Förster, A., Großmann, J., Meyer, R., Reinicke, K., Schäfer, D., and Wendel, H. (2011). CLEAN: Preparing for a CO2-based Enhanced Gas Recovery in a Depleted Gas Field in Germany. Energy Procedia 4, pages 5520-5526. doi: 10.1016/j.egypro.2011.02.538.

Kühn, M., Tesmer, M., Pilz, P., Meyer, R., Reinicke, K., Förster, A., Kolditz, O., and Schäfer, D. (2012). CLEAN: project overview on CO2 large-scale enhanced gas recovery in the Altmark natural gas field (Germany). Environ. Earth Sci. doi: 10.1007/s12665-012-1714-z.

Levander, A. R. (1988). Fourth-order finite-difference P-SV seismograms. Geophysics, 53 (11), 1425-1436.

Liu, H. P., Anderson, D. L., and Kanamori, H. (1976). Velocity dispersion due to anelasticity: Implications for seismology and mantle composition. Geophys. J. Roy. Astr. Soc., 47, 41-58.

Lumley, D. (2010). 4D seismic monitoring of $\mathrm{CO}_{2}$ sequestration. The Leading Edge, 29, 150-155.

Lüth, S., Buske, S., Goertz, A., and Giese, R. (2005). Fresnel-volume migration of multicomponent data. Geophysics, 70 (6), 121-129.

Ma, J. and Morozov, I. (2010). AVO modeling of pressure-saturation effects in Weyburn $\mathrm{CO}_{2}$ sequestration. The Leading Edge, 29, 178-183.

Martens, S., Kempka, T., Liebscher, A., Lüth, S., Möller, F., Myrrtinen, A., Norden, B., Schmitt-Hattenberg, C., Zimmer, M., Kühn, M., and Group, T. K. (2012). Europe's longest-operating on-shore $\mathrm{CO}_{2}$ storage site at Ketzin, Germany: a progress report after three years of injection. Environmental Earth Sciences. doi:10.1007/s12665-012-1672-5. 
Mathieson, A., Midgley, J., Dodds, K., Wright, I., Ringrose, P., and Saoul, N. (2010). $\mathrm{CO}_{2}$ sequestration monitoring and verification technologies applied at Krechba, Algeria. The Leading Edge, 29, 216-222.

Mavko, G., Mukerji, T., and Dvorkin, J. (1998). The Rock Physiks Handbook. Cambridge University Press, Cambridge, UK.

Murphy, W. F. I. (1982). Effects of partial water saturation on attenuation in Massilon sandstone and Vycor porous glass. J. Acoust. Soc. Am., 71, 1458-1468.

NETL (2007). Carbon Sequestration Technology and Program Plan 2007. Roadmap, National Energy Technology Laboratory (NETL) and U.S. Department of Energy, USA.

Newman, P. (1975). Amplitude and phase properties of a digital migration process. In 37th Annual International Meeting. EAGE. republished in 1990, First Break,8 (11), pages 397-403.

Poupinet, G., Ellisworth, W. J., and Frechet, J. (1984). Monitoring velocity variations in the crust using earthquake doublets: an application to the Calaveras Fault, California. J. of Geophys. Res., 89 (B7), 5719-5731.

Radgen, P., Cremer, C., Warkentin, S., Gerling, P., May, F., and Knopf, S. (2006). Verfahren zur $\mathrm{CO}_{2}$-Abscheidung und -Speicherung. Abschlussbericht 07/06, Federal Ministry for the Environment, Nature Conservation and Nuclear Safety, Dessau, Germany.

Rebscher, D. and Oldenburg, C. M. (2005). Sequestration of Carbon Dioxide with Enhanced Gas Recovery - Case Study Altmark, North German Basin. Report lbnl-59033, Lawrence Berkley National Laboratory, Berkeley, California, USA.

Reinhold, K., Müller, C., and Riesenberg, C. (2011). Informationssystem Speichergesteine für den Standort Deutschland - Synthese. Abschlussbericht, Federal Institute for Geosciences and Natural Resources (BGR), Berlin/Hannover, Germany.

Robertsson, J. O. A., Blanch, J. O., and Symes, W. W. (1994). Viscoelastic finitedifference modeling. Geophysics, 59 (9), 1444-1456.

Rückheim, J., Voigtländer, G., and Stein-Khokhlov, M. (2005). The Technical and Economical Challenge of "Mature Gas Fields": The Giant Altmark Field, a German Example. SPE Europec/EAGE Annual Conference, 13 -16 June 2005. 
Sayers, C. and Wilson, T. (2010). An introduction to the special section: $\mathrm{CO}_{2}$ sequestration. The Leading Edge, 27 (2), 148-149.

Scheck-Wenderoth, M. and Lamarche, J. (2005). Crustal memory and basin evolution in the Central European Basin System - new insights from a 3D structural model. Tectonophysics, 397, 143-165.

Schleicher, J., Tygel, M., and Hubral, P. (1993). 3-D true-amplitude finite offset migration. Geophysics, 58, 1112-1126.

Schröder, L. (1989). Erdöl und Erdgas in Niedersachsen, In: Rohstoffvorkommen in Niedersachsen - Wirtschaftliche Bedeutung und Abbaumöglichkeiten. Nds. Akad. Geowiss. Veröfftl., 3, 32-42.

Setzmann, U. and Wagner, W. (1991). A New Equation of State and Tables of Thermodynamic Properties for Methane. J. Phys. Chem. Ref. Data, 20 (6), 1061-1151.

Shapiro, S. A. (2003). Elastic piezosensitivity of porous and fractured rocks. Geophysics, 68, 482-486.

Sick, C. (2005). Structural investigations of Chile: Kirchhoff prestack depth migration versus Fresnel-Volume migration. Ph.D. thesis, Institute of Geological Sciences, Freie Universität Berlin. URL: http://www.diss.fu-berlin.de.

Snieder, R., Grét, A., Douma, H., and Scales, J. (2002). Coda wave interferometry for estimating nonlinear behavior in seismic velocity. Science, 295.

Span, R. and Wagner, W. (1996). A New Equation of State for Carbon Dioxide covering the Fluid Region from the Triple-Point Temperature to $1100 \mathrm{~K}$ at Pressures up to 800 MPa. J. Phys. Chem. Ref. Data, 25 (6), 1509-1596.

Span, R., Lemmon, E. W., Jacobsen, R. T., Wagner, W., and Yokozeki, A. (2000). A Reference Equation of State for the Thermodynamic Properties of Nitrogene for Temperatures from 63.151 to $1000 \mathrm{~K}$ and Pressures to $2200 \mathrm{MPa}$. J. Phys. Chem. Ref. Data, 29 (6), 1361-1433.

Spencer, J. W. J. (1981). Stress relaxations at low frequencies in fluid-saturated rocks: attenuation and modulus dispersion. J. Geophys. Res., 86, 1803-1812.

Suprajitno, M. and Greenhalgh, S. A. (1985). Separation of upgoing and downgoing waves in vertical seismic profiling by contour-slice filtering. Geophysics, 50 (6), 950-962. 
Thomas, L. K., Hankinsin, R. W., and Phillips, K. A. (1970). Determination of Acoustic Velocities for Natural Gas. J. Petr. Tech., 22, 889-892.

UNFCCC (2011). National Greenhouse Gas Inventory Database for the period 1990-2009. Data base, United Nations Framework Convention on Climate Change (UNFCCC). URL: http://ghg.unfccc.int;download 16.11.2011.

Urosevic, M., Pevzner, R., Shulakova, V., Kepic, A., Caspari, E., and S., S. (2011). Seismic monitoring of $\mathrm{CO}_{2}$ injection into a depleted gas reservoir - Otway Basin Pilot Project, Australia. Energy Procedia, 4, 3550-3557.

Verdon, J. P., Kendall, J.-M., White, D. J., Angus, D. A., Fisher, Q. J., and Urbancic, T. (2010). Passive seismic monitoring of carbon dioxide storage at Weyburn. The Leading Edge, 29, 200-206.

Virieux, J. (1986). P-SV wave propagation in heterogeneous media: velocity-stress finite-difference method. Geophysics, 51 (4), 889-901.

Walls, J. and Dvorkin, J. (2005). Effects of Pore Fluid Properties at High Pressures and Temperatures on Seismic Response. In 2005 SEG Annual Meeting, November 6 - 11, 2005 , Houston, Texas.

Welfens, P. J. J., Meyer, B., Pfaffenberger, W., Jasinski, P., and Jungmittag, A. (1999). Towards a re-orientation of national policies in the EU? - Germany as case study. Energy and research series, European Parliament.

Wendel, H. (2007). The Altmark gas field (Germany): Investigating CCS Potential under an EGR Project. In 2nd International Symposium - Capture and geological storage of CO2, Paris, 3 - 5 October 2007. URL: http://www.co2symposium.com/2007/program_va.htm.

White, C. M., Strazisar, B., Granite, E. J., Hoffman, J. S., and Pennline, H. W. (2003). Separation and capture of $\mathrm{CO}_{2}$ from large stationary sources and sequestration in geological formations - coalbeds and deep saline aquifers. Journal of the Air \& Waste Management, 53, 645-715.

White, D. (2009). Monitoring $\mathrm{CO}_{2}$ storage during EOR at the Weyburn-Midale Field. The Leading Edge, 28, 838-842.

Wilson, M. and Monea, M. (2004). IEA GHG Weyburn $\mathrm{CO}_{2}$ monitoring and storage project. summary report 2000-2004, Petroleum Technology Research Centre (PTRC). URL: http://www.ptrc.ca/weyburn_first.php. 
Xue, Z. and Ohsumi, T. (2004). Seismic wave monitoring of $\mathrm{CO}_{2}$ migration in water-saturated porous sandstone. Exploration Geophysics, 35, 25-32.

Zhou, R., Huang, L., Rutledge, J. T., Fehler, M., Daley, T. M., and Majer, E. L. (2010). Coda-wave interferometry analysis of time-lapse VSP data for monitoring geological carbon sequestration. International Journal of Greenhouse Gas Control, 4, 679-686.

Zimmermann, R. W., Somerton, W. H., and King, M. S. (1986). Compressibility of porous rocks. J. Geophys. Res., 91, 12765-12777. 



\section{Appendix A}

\section{Comparison of equations of state}

In literature a large number of equations of state are existing for methane. Two widely used equations, the first from Batzle and Wang (1992) and the second from Setzmann and Wagner (1991) are compared here.

Batzle and Wang start from the ideal gas law

$$
V_{\text {mol }}=\frac{R T}{P}
$$

where $V_{\text {mol }}$ is the molar volume, $\mathrm{R}$ is the gas constant, $\mathrm{T}$ is the temperature in Kelvin and $\mathrm{P}$ is the pressure.

As methane is not an ideal gas, Batzle and Wang include the compressibility factor $\mathrm{Z}$ in the ideal gas law

$$
V_{m o l}=\frac{Z R T}{P}
$$

to better approximate the real behaviour of methane. Furthermore it must be considered, that the propagation of acoustic waves in a medium is an adiabatic process. These two assumptions lead to the following equations for density

$$
\rho=\frac{M_{m o l}}{V_{m o l}}=\frac{M_{m o l} P}{Z R T}
$$

and adiabatic bulk modulus

$$
K_{S}=\frac{1}{\beta_{S}}=\frac{\gamma P}{\left(1-\frac{P}{Z} \frac{\delta Z}{\delta P}\right)_{T}},
$$

where $M_{m o l}$ is the molecular weight, $\gamma$ is the ratio of the isobaric and the isochoric heat capacity and gives the correlation of the isothermal and adiabatic compressibility

$$
\beta_{T}=\gamma \beta_{S}=\frac{c_{p}}{c_{V}} \beta_{S} .
$$


In most realistic cases one did not have pure hydrocarbon gases but mixtures of different natural gases. To make the calculation of the properties of mixtures more systematic, Batzle and Wang normalized pressure and temperature by the pseudocritical values, which are the weighted molar average of the pure compounds. Thomas et al. (1970) found a relation to calculate these then called pseudoreduced values by the gravity $\mathrm{G}$ of the gas composition.

$$
P_{p r}=\frac{P}{P_{p c}}=\frac{P}{4.892-0.4048 G}, \quad T_{p r}=\frac{T}{T_{p c}}=\frac{T}{94.72-170.75 G},
$$

where $\mathrm{P}$ is in MPa.

Now the gas properties can be calculated if the compressibility factor and the ratio of heat capacities $\gamma$ is known. These can be calculated by the Benedict-WebbRubin (BWR) equation of state.

For typical temperature and pressure ranges, that occur in gas and oil exploration, Batzle and Wang used the approximations

$$
\rho=\frac{28.8 G P}{Z R T}
$$

and

$$
K_{S}=\frac{\gamma_{0} P}{\left(1-\frac{P_{p r}}{Z} \frac{\delta Z}{\delta P_{p r}}\right)_{T}},
$$

with

$$
\begin{aligned}
Z= & {\left[0.03+0.00527\left(3.5-T_{p r}\right)^{3}\right] P_{p r}+\left(0.642 T_{p r}-0.007 T_{p r}^{4}-0.52\right) } \\
& +0.109\left(3.85-T_{p r}\right)^{2} e^{-\left[0.45+8\left(0.56-\frac{1}{T_{p r}}\right)^{2}\right] \frac{P_{p r}}{T_{p r}}}
\end{aligned}
$$

and

$$
\gamma_{0}=0.85+\frac{5.6}{P_{p r}+2}+\frac{27.1}{\left(P_{p r}+3.5\right)^{2}}-8.7 e^{-0.65\left(P_{p r}+1\right)} .
$$

These equations are a good approximation for $0.1<T_{p r}<1$ and $0.1 \leq P_{p r} \leq 1$ and are a simple way to calculate the properties of hydrocarbon gas compositions. But for pure methane Batzle and Wang found differences of several percent between calculated and measured velocities, which result from errors in the calculation of the volume in the BWR equation by Thomas et al. (1970).

Setzman and Wagner went a very different way. They selected a huge set of experimental data of all thermodynamic properties of methane up to the middle of 1991. This dataset included thermal properties of the single phase and of the liquidvapor saturation curve including the Maxwell criterion, speed of sound, isochoric and isobaric heat capacity, difference of enthalpy and second virial coefficient and covers 
a region of $90.6941 K \leq T<625 K$ and $0.011696 M P a \leq p \leq 1000 M P a$. Also other independent equations describing the phase transitions as well as saturated liquid and vapour densities and isobaric ideal gas heat capacity were included.

Based on this dataset, Setzmann and Wagner developed an equation for the dimensionless Helmholtz energy $\Phi$, that can be split in two parts, one depending on the ideal gas behaviour $\Phi_{0}$ and another one depending on the residual fluid behaviour $\Phi_{r}$

$$
\Phi(\delta, \tau)=\frac{A(\delta, \tau)}{R T} \Phi^{0}(\delta, \tau)+\Phi^{r}(\delta, \tau)
$$

with the Helmholtz energy $A(\delta, \tau)$ and the reduced density $\delta=\frac{\rho}{\rho_{c}}$ and the inverse reduced temperature $\tau=\frac{T_{c}}{T}$.

The Helmholtz energy of the ideal gas is calculated by means of an equation for the ideal gas heat capacity and is valid for the temperature region of $60 K \leq T \leq$ $3000 \mathrm{~K}$.

To obtain the function for the residual part of the Helmholtz equation Setzmann and Wagner implemented a three step fitting and optimizing procedure. In a first step they formulated a general expression for the equation. Afterwards, in the second step, different forms of equations are fitted to the linear and linearized experimental data by minimizing the weighted sum of squares

$$
\chi^{2}=\sum_{j=1}^{J} \chi_{j}^{2}=\sum_{j=1}^{J} \sum_{m=1}^{M_{j}}\left[\frac{\left[z_{\exp }-z\left(\Phi, \delta_{\exp }, \tau_{\text {exp }}, n\right)\right]^{2}}{\sigma_{\text {exp }}^{2}}\right]_{j, m}
$$

with $\mathrm{z}$ as the general thermodynamic variable $\left(p, h, c_{v}, c_{p}, \ldots\right)$ and $\sigma$ the total uncertainty of the particular experimental data. Not all thermodynamic properties are related linear to the Helmholtz function, which is a function of temperature and density $A(\delta, \tau)$. Some properties were measured in dependence of pressure and temperature as it is the case for enthalpy $h(T, p)$, isobaric heat capacity $c_{p}(T, p)$ and speed of sound $v(T, p)$. They have to be linearized in the variables of the Helmholtz function before running the optimization procedure. In step three a nonlinear fitting process is performed to take also the original experimental information of the nonlinear data into account. With this optimized equation the precalculated data for the fit of the linearized data were calculated and step two and three are repeated until a convergence criterion is met.

With the so found Helmholtz equation all properties of methane can be calculated. The $p \rho T$ behaviour is given by

$$
\frac{p(\delta, \tau)}{\rho R T}=1+\delta \Phi_{\delta}^{r}
$$


and the speed of sound by

$$
\frac{v^{2}(\delta, \tau)}{R T}=1+2 \delta \Phi_{\delta}^{r}+\delta^{2} \Phi_{\delta \delta}^{r}-\frac{\left(1+\delta \Phi_{\delta}^{r}-\delta \tau \Phi_{\delta \tau}^{r}\right)^{2}}{\tau^{2}\left(\Phi_{\tau \tau}^{0} \Phi_{\tau \tau}^{r}\right)}
$$

where the subscripts of the Helmholtz function denote the deviation with respect to the particular quantity.

The validity of the function covers a range from the melting curve to $625 \mathrm{~K}$ at pressures up to $1000 \mathrm{MPa}$. In this region the uncertainty of density is less than $0.15 \%$, for temperatures less than $500 K$ even smaller than $0.07 \%$. For speed of sound the uncertainty is less than $0.5 \%$ and only in the direct vicinity of the critical point it goes up to $5 \%$.

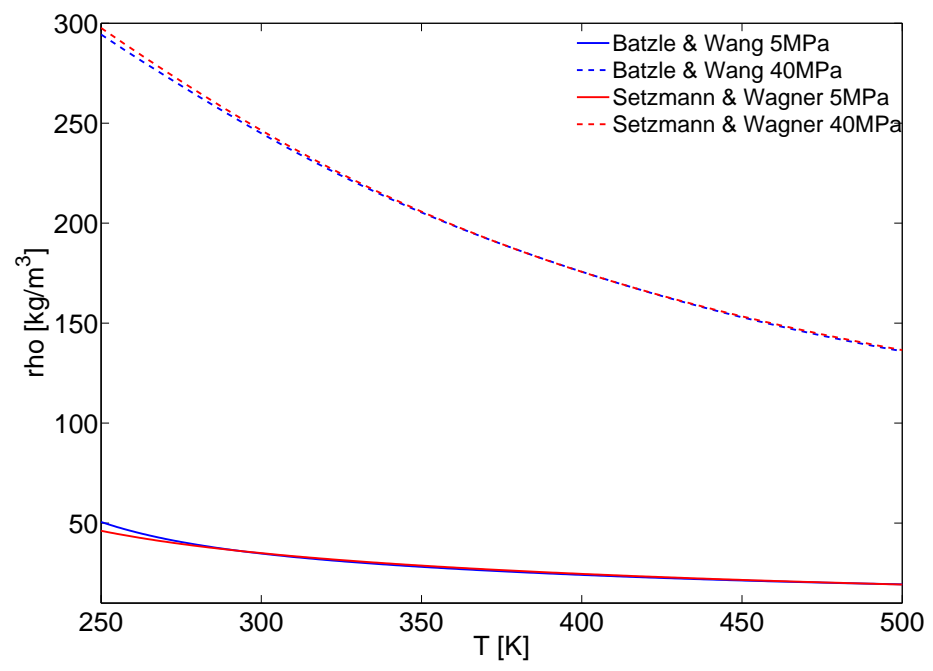

Figure A.1: Density of methane over temperature calculated after Batzle and Wang (1992) and Setzmann and Wagner (1991) for pressures of $5 M P a$ and $40 M P a$, respectively. The two formula show good agreement over the whole temperature range and for the observed pressures.

The comparison of the results of Batzle and Wang (1992) and Setzmann and Wagner (1991) shows that the difference in density is small for the temperature range of $250 \mathrm{~K}$ to $500 \mathrm{~K}$ and pressures from $4.6 \mathrm{MPa}$ to $40 \mathrm{MPa}$ (figure A.1). But the bulk modulus and with it the speed of sound differs significantly by calculating with either of the functions. At $5 \mathrm{MPa}$ and $400 \mathrm{~K}$, which are the conditions of the Altmark reservoir, the difference of the bulk modulus is more than $20 \%$. This results in a miscalculation of the speed of sound of more than $11 \%$ (figure A.2). These results are also shown by other authors (Walls and Dvorkin, 2005). Due to the inaccuracies of the equation by Batzle and Wang we decided to calculate the gas properties of methane from the mathematically more complex, but more exact equation of Setzmann and Wagner. Wagner applied this method on a large number 

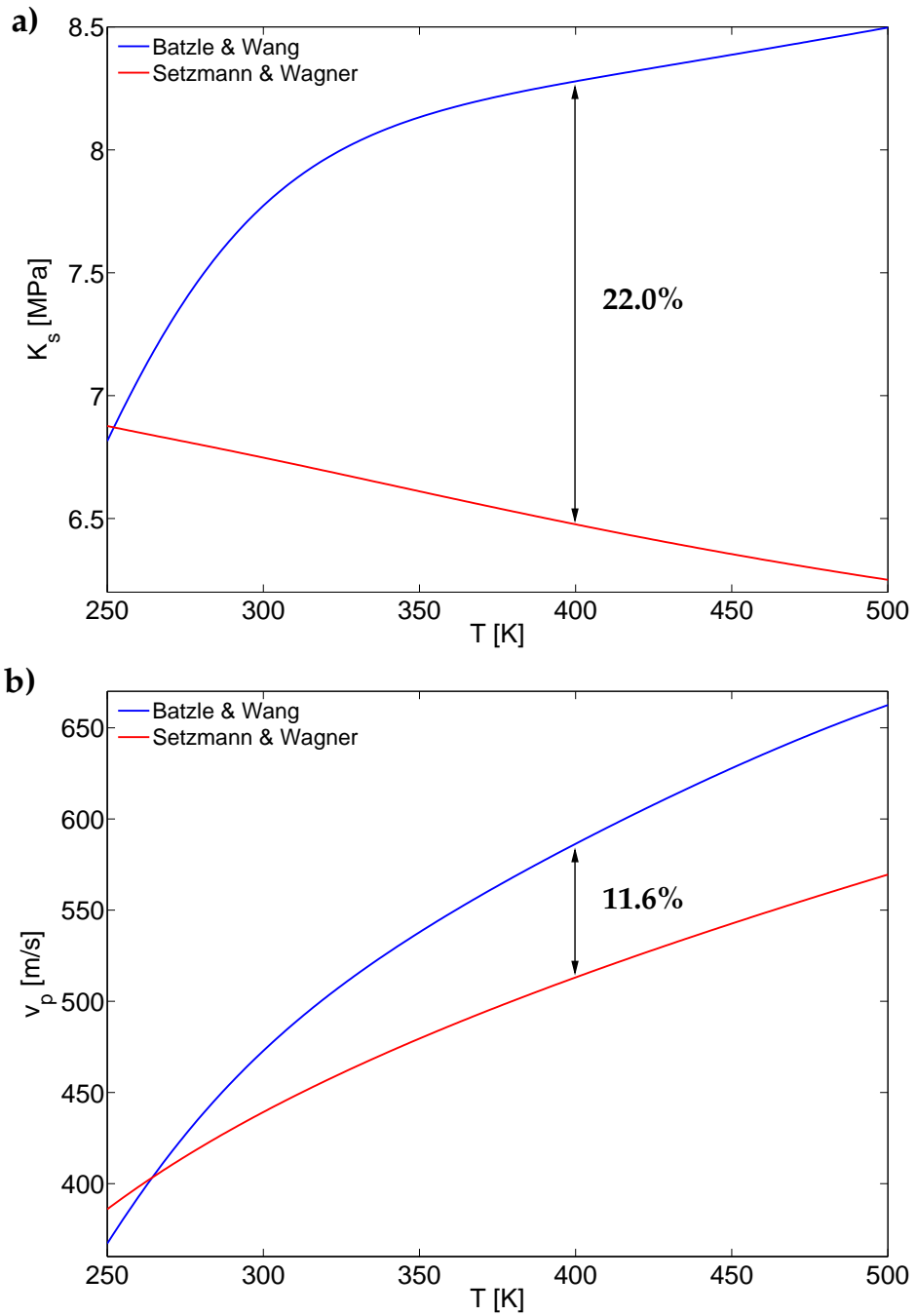

Figure A.2: a) bulk modulus and b) velocity of methane over temperature calculated after Batzle and Wang (1992) and Setzmann and Wagner (1991) for pressures of $5 \mathrm{MPa}$. They show large differences for temperatures of more than $300 \mathrm{~K}$. 
of other gases. We also used their equations of state to calculate density and velocity of nitrogen (Span et al., 2000) and carbon dioxide (Span and Wagner, 1996), the other two gases that are relevant for the modelling. 


\section{Acknowledgements}

The project CLEAN has been funded within the framework of the GEOTECHNOLOGIEN program by the Federal Ministry of Education and Research (BMBF). We gratefully acknowledge GDF SUEZ E\&P Deutschland GmbH for their support and for providing the data used in this study.

I thank my supervisor Prof. Dr. Stefan Buske for the mentoring, support and constructive discussions during these years and Prof. Dr. Serge Shapiro for his advise.

Further I thank Dr. Michaela Bock for her relendless dedications to make the best of an impossible mission.

I thank Dr. Juliane Kummerow and Dr. Pudlow for their assistance and the supply of the rock sample data used in my thesis.

I thank all the friends and colleagues at the Freie Universität Berlin that gave advise, discussed or helped me to clear my mind with playing football. In this context I would like to mention especial Dr. Cedric Schmelzbach and Dr. Jörn Kummerow.

And I thank of course all the committed proof-readers Christopher Wollin, Thomas Partridge, Andrew Irving, Astrid Houpt and Rolf Houpt who let me look as if I could speak english.

I thank my brother and sisters for cheering me up when it was necessary. I thank my parents, who made it possible for me to get where I am now. Finally I thank my wife and my kids for their support, their understanding and their encouragement. 